\title{
Clarifying tetrapod embryogenesis, a physicist's point of view
}

\author{
V. Fleury ${ }^{\mathrm{a}}$ \\ CNRS/Institut de Physique de Rennes, Université de Rennes 1, 35042 Rennes, France
}

Received: 10 June 2008 / Accepted: 26 January 2009

Published online: 11 March 2009 - (c) EDP Sciences

\begin{abstract}
The origin of tetrapods is a complex question that webs together genetic, paleontological, developmental and physical facts. Basically, the development of embryos is described by a complex mix of mechanical movements and biochemical inductions of genetic origin. It is difficult to sort out in this scientific question what are the fundamental features imposed by conservation laws of physics, and by force equilibria, and what can be ascribed to successive, very specific, stop-and-go inductions of genetic nature. A posteriori, evolution selects the parameters of this process as found in the observed species. Whether there is a general law to animal formation seems out of the question. However, several concepts developed in biology, like the concept of "organizer" seem questionable from a physics point of view, since the entire deformation and force field should be the "organizer" of development, and one can hardly ascribe such a role to a single small area of the embryo body. In the same spirit, the concept of "positional information" encapsulated in concentration of chemicals seems questionable since the deformation and force fields in embryonic tissues are tensors. Finally, the concept of a development organized in space along three orthogonal ("Cartesian") axes associated to chemical gradients seems also questionable, since early embryo development is driven by complex vortex fields, with hyperbolic trajectories which span the entire embryo. Such hyperbolic trajectories are best understood by a description in terms of dipolar components of the morphogenetic forces, whose projections along orthogonal axes have no specific meaning except as a mathematical tool. I review here the present state of description of several aspects of tetrapods morphogenesis and evolution, from the point of view of physics. It is getting clear that several basic features of tetrapods body are a direct consequences of fundamental laws of physics. Several lines of work reviewed here show that the topology of the tetrapods may be directly related to the structure of the earliest movements in embryos. The bio-mechanical approach leads to important consequences for the constraints on evolution of the craniates. Such consequences have received a controversial welcome in the last decade, although they may encapsulate the true origin of craniates, esp. simians, and eventually homo.
\end{abstract}

PACS. 87.19.lx Development and growth - 87.18.-h Biological complexity - 68.15.+e Liquid thin films

\section{Introduction}

There is no need to explain that animal embryos, especially vertebrates, are material "things". As such they develop following the laws of condensed matter physics, especially Newton's law for deformations (elasticity laws) and deformation rates (viscous materials). A complete description of animal body formation should end by finding the entire morphogenetic field. An animal $A_{i}$ should be written in terms of its fertilized ovocyte $\mathrm{O}_{\mathrm{i}}$ and the displacement rate vector $\mathrm{u}_{\mathrm{i}}$ :

$$
A_{i}(x, y, z, T)=O_{i}(x, y, z)+\int_{0}^{T} u_{i}(x, y, z, t) d t .
$$

a Present address: Laboratoire Matière et Systèmes Complexes, Université de Paris VII-Denis Diderot, 75013 Paris, France.

e-mail: vincent.fleury@paris-diderot.fr
One may expect the field $u_{i}(x, y, z, t)$ to contain all the physical parameters of genetic origin. Understanding the development of an animal requires then to find the displacement rate field, as a function of all chemicals in the problem, which may seem a formidable task. In order to do so, a classical approach in physics consists in finding the relationship between deformation rates, stresses and volume forces, and encapsulate this relationship into a constitutive equation for the said material.

The genetic approach is completely different. It is assumed that genes, being the true parameters of the developmental process, determine completely animal traits, and that finding the entire set of genes playing a role in the problem, and the way they influence each other, should clarify the problem of animal development. Since genes may be altered or duplicated during cell division mishaps (leading eventually to new species), and since modification of genes may modify the complex set of inductive actions, a precise description of body formation may require 
a fantastic analysis of lots of genetically chained reactions. In this spirit, there would exist a few master genes which organize body axis, like bicoid or nanos, and homeotic Hox genes [1], which influence other genes which are found "downstream", and determine the exact shape of localized body parts by chemical modification of proteins (either concentrations, timing or interactions). Also, due to hierarchical construction of the genetic reaction networks, there would exist specific robust genetic regulatory kernels, which are less likely to evolve during evolution $[2,3]$.

Understanding gross morphological traits would consist in understanding the interplay of the main master genes, before going into more detailed features (e.g.: having feathers or hairs, hooves or nails, etc.). A considerable activity exists in this field of developmental biology, especially in relation to the fly model drosophila, but it is getting increasingly evident that fly morphogenesis is quite different from vertebrate morphogenesis.

In terms of animal description, paleontology lies in between physics and genetics. Indeed, in many cases paleontologists are able to describe the transition from one form to another, by analyzing in detail shifts, or drifts, in body parts, e.g. neck or tail elongation [4], or transition from plantigrades (animals walking on the sole, like humans) to digitigrades (animals walking on the fingers, like dogs) [5] (Fig. 1), but it is very rare that direct genetic data may have been preserved in fossils, except the latest ones [6].

Therefore, genetic analysis of developmental shifts of traits between extinct species is difficult. Shifts of traits as observed on species are ascribed to selection of improved traits, in terms of adaptation, but traits should be able to shift, in the first place. In this rationale, traits appear first in a small group of animals, possibly one single individual, and then spread by Darwinian selection of the fittest, with possible subtleties like accelerated selection in geographically isolated groups of individuals (punctuated equilibrium, etc.) [7]. There exist bitter controversies about the dynamics of natural selection $[8,9]$. However, the paleontological description of how traits actually appear, before being selected, is interesting from the point of view of physics: anatomical traits seem to "derive" or "drift" from previous traits. Folds seem to "extend", joints "rotate", body parts "expand", vestigial organs progressively "reduce". This wording assumes implicitly some mechanical feature, and the existence of dimensionless traits which can be scaled up or down, wound and rewound, elongated or stretched arbitrarily, in a space of latent forms.

It has long been recognized, for example in the classical observations of D'Arcy Thomson [10] (Fig. 2), that animal forms may often be obtained one from each other by seemingly simple distortion maps, i.e., by a simple integral over an abstract space-time "on paper" of a physical deformation rate field. This is to say that, although two animal species are formed of different individuals, which developed in different eggs or wombs at different times, it seems that one can construct an abstract geometrical space in which species forms can be deformed into one an-
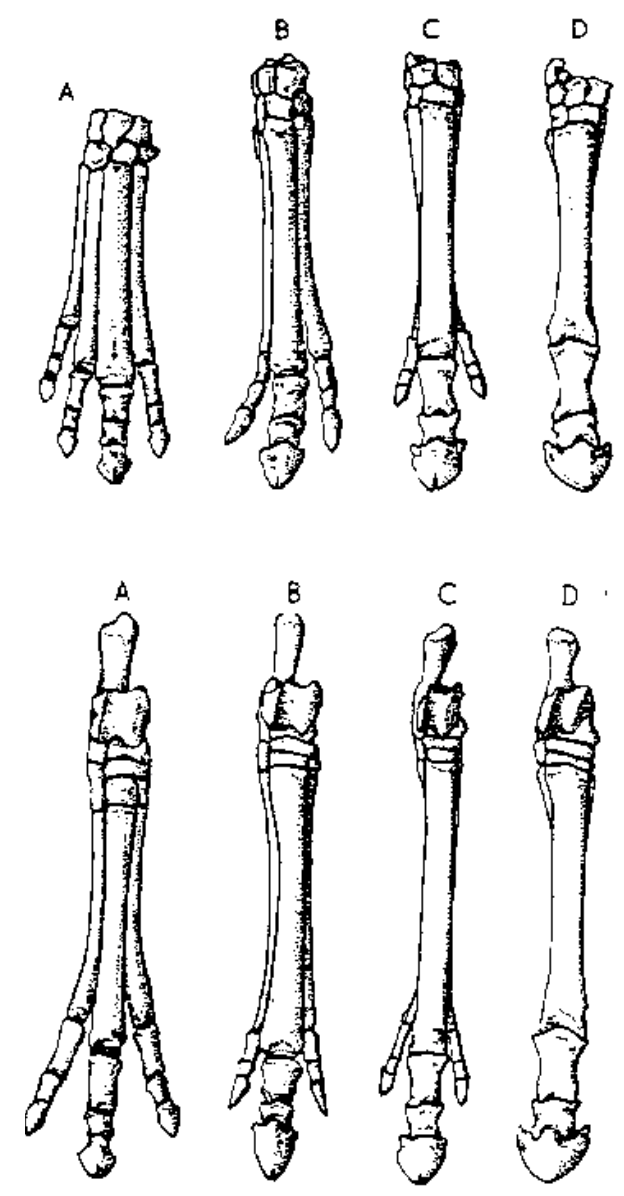

Fig. 1. Transition from digitigrades to unguligrades ${ }^{1}$. The plate represents the bone structure of animals as found in the fossil record, with transitional forms from plantigrades to digitigrades and unguligrades (From Ref. [5]). The fossil records shows animal species with progressive limb extension, these extensions correlate with reduction of lateral fingers. The lateral fingers may remain as vestigial, almost useless phalanges.

other by formal spatio-temporal mechanical fields in the space of shapes.

Therefore, there is a possibility that animal formation may be understood on the basis of physical principles in the following way: genetic parameters, and their inductive relationships set a number of mechanical properties, such as material constants, instability parameters and volume forces, then the integral during development in each individual of the deformation rate tensor gives the animal shape at birth. A modified individual shape corresponds to a different set of input genetic parameters, and selection acts a posteriori on the modified shape to stabilize the species and maintain it over a significantly long time, such that the species may be present in the fossil record at all. Consider two animals belonging to different species,

\footnotetext{
1 It is interesting to note that there exists even in human a condition called "equine walk", by which children, and even adults have a digitigrade walk. In some instances this condition is related to tendon shortening. In other cases, no particular pathology is found, and these patients just walk like that.
} 


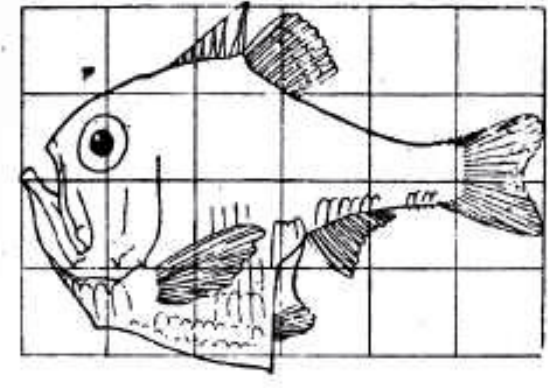

Fig. 517. Argyropelecus Olfersi.

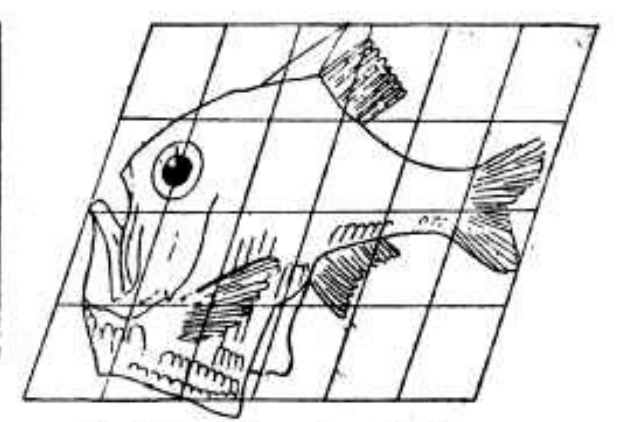

Fig. 518. Sternoptyx diaphana.

Fig. 2. Anamorphosis maps proposed by d'Arcy Thomson. From On growth and form [10]. D'Arcy Thomson had the intuition that different animal forms could be related by simple macroscopic deformations acting on the same qualitative pattern (stated otherwise, a "dimensionless" archetypic animal can be mapped onto other species by affine transformations).

if they are formed by the same developmental process, although with different biomechanical parameters, they will still be related by apparent simple deformations, since the developmental process has a dimensionless form, rescaled by the said parameters.

A natural question arises then, whether this process of animal formation can be described by a general law of condensed matter physics, with a given set of parameters, such that general scaling laws may be found resting on few parameters, and if yes, how many such parameters? At present, the count of main master genes is 39 [11]. However, it is obvious from simple inspection that limbs may extend during evolution, although retaining the general "scaffold" of the limb. This is indeed exactly what was recognized by Charles Darwin [12]. Especially, he expected the forelimb of the mouse to become the wing of the bat, "by extending the bones, but without changing in any way the scaffold" [13]. Nowadays, gene promoters have been found in the bat which, once transferred to the mouse, are indeed able to extend the mouse limb by, say, $6 \%$ [14]. The authors in reference [14] consider that the existence of such a promoter of the gene (Prx1) is a proof of the Darwinian theory of natural selection. However, it is an extraordinary fact, unexplained by Darwin, that the limb of a mouse can extend directly, in a straightforward manner, by modifying a single gene promoter. When Darwin states that limbs extend by "conserving the scaffold", he means that all the other erroneous plans that may have emerged were put off by selection. Out of context, the original sentence is quite ambiguous ${ }^{2}$. For Darwin, the straightforward character of the wing evolution is an illusion due to disappearance by selection of all the other options. Darwinian evolution is not just a matter of slow changes of body forms, it is a matter of slow changes, se-

\footnotetext{
2 "In changes of this nature, there will be little or no tendency to modify the original pattern, or to transpose parts. The bones of a limb might be shortened and widened to any extent, and become gradually enveloped in thick membrane, so as to serve as a fin; or a webbed foot might have all its bones, or certain bones, lengthened to any extent, and the membrane connecting them increased to any extent, so as to serve as a wing". (ibid. Chap. XIII).
}

lected among an infinite set of possible, but not selected, shapes. More specifically, Darwin does not ascribe the conservation of the "scaffold" to some fundamental physical constraint, but to the fact that the evolution is incremental. His statements on this issue are unclear, because he stresses properties of species, as they actually exist after selection, without addressing the morphogenetic process itself, which occurs in embryos ${ }^{3}$. In the same spirit, when paleontologists show how bowing prehominians progressively stand more upwards [15], they do not expect this tendency to be a straightforward possibility but rather the retrospective illusion due to disappearance of all alternatives. Actually, the existence of a bat gene that, transferred to mice, extends their limbs, is not a true proof of Darwinian evolution, as long as all the other possible outcomes, which were not selected, are not shown. Unfortunately, since aberrant mutations do not form species it is hard to tell whether the gene associated to the limb elongation was actually selected among many others, or whether limbs cannot do anything else but elongate or shorten.

In particular, when Charles Darwin states explicitly (ibid. Chap. XIII) that limb bones can be stretched or flattened in arbitrary proportions, he fails to recognize two important facts. First, that this corresponds to affine transformations only, and, in the absence of morphogenetic constraints, there is no a priori reason to exclude other deformation modes, and, more importantly, that flattening in one direction can be correlated to extension in the other by mechanical laws, thereby reducing the number of parameters in the problem. Also, it is an obvious fact that limbs can be stretched in enormous proportions, while no animal has a femur with a diameter as large as a bat wing is long. In addition to size change, the number and distribution of bones inside limbs may be modified

\footnotetext{
3 In Darwin's book, physical morphogenesis is sometimes evoked as the source of "variability". When searching for the causes of morphogenetic "variability", Darwin in a few cases comes to the conclusion that physical forces, and especially pressure gradients, may drive morphogenetic variability, and internal shape modifications. When this happens, he states, natural selection plays no role in the observed form.
} 
with subtle mode-locking effects, such as reduction of phalanx count. Such correlations may not be identified as a simple consequence of limb extension, but be ascribed to complex genetic inductions, or to selection pressure.

It comes as a new feature of animal morphogenesis, that the animal parts may be physical entities which may be elongated or shortened, by direct modification of genes, without changing at all the pattern, in its dimensionless form. The origin of this phenomenon lies in the existence of streamlines inside the embryo body, these streamlines confine cell paths, and hence animal modification by modification of cellular forces have to follow the streamlines [16]. This suggests that, although complex 3D patterns, the animal body parts, and possibly the entire animal, may be physical objects deformable by the action of very few, and in some areas of the body, only one degree of freedom, onto which a considerable number of genes are mapped. This is apparent in the existence of mutations which cause for example dwarfism [17], or gigantism [18]. It is also apparent in the fact that, when a genetic cause is searched for a given morphological abnormality or pathology, it is very rare that a single molecular defect is identified: several molecular causes induce similar morphogenetic effects. In the same spirit, also, evolutionary convergence, i.e. morphological homologies between distant species, are not ascribed to induction of identical pathways in different genera, but to common macroscopic morphogenetic processes, induced by completely different means.

How all this may be possible is related to the fact that animal formation is a matter of physical deformation of an early fertilized egg, which is basically formless (round), and the deformation modes of a round material into an elongated one (for the case of bilaterians) have a finite typology.

In the sequel, I shall review in more detail the genetic description of formation of animal bodies, starting by the fly, and then exposing the case of the vertebrates (Sects. 2 and 3). Then, I shall review the paleontological data (Sect. 4) dealing with the appearance of the vertebrate body plan. I will stress on facts more relevant for our purpose, which is to highlight how biomechanical fields of tissue deformation and of cell orientation may constrain body shapes and evolution.

However, the genetic and paleontological issues do not allow one to truly understand how an animal is actually constructed. Therefore, I next review the developmental data about the actual formation of the vertebrate body plan (Sect. 5), with a focus on embryo movements, and on the "limb field". This leads us to a description of the embryo morphogenetic movements, which appear quite simple, actually. These movements are so simple that, independently of the genetic substrate, physics can already say enough about this phenomenon to make a few qualitative, but important, predictions about the body organization, the number and positioning of tetrapods limbs, and the tendencies of the morphogenetic dynamics ${ }^{4}$. The physical

\footnotetext{
${ }^{4}$ It is generally thought in developmental biology that there does not exist a unified description of vertebrate development, and that different animals, for example, chickens, frogs and pri-
}

description is thus treated in part 6 , for the general aspects, and 7 for a more detailed application to tetrapod embryogenesis. Section 8 is a short section devoted to the question of segmentation. Section 9 discusses the consequences of tissue expansion in the case of the craniates.

In conclusion (Sect. 10), and returning to current controversies in paleontology, I show that a very simple scenario of body plan formation emerges, which may explain, on mathematical grounds, the origin of these animals, and which sheds some light on the constraints of Darwinian evolution by natural selection. The formation of this body plan can be qualitatively boiled down to a simple hyperbolic flow as I have proposed in references $[16,119]$. This flow generates a "generic" or "archetypic" tetrapod. Actually, such an archetype is explicitly invoked by Charles Darwin. In the Origin of species, Charles Darwin explains that all animals are very much alike, and that he expects all animal forms to derive from a small set of about 4 or "at most" 5 typical plans, called "archetypes" 5 or "prototypes" 6 , (ibid. Chap. XIV). According to Darwin, the apparent variety of forms is obtained, as for the bat wing, by extensions of a general pattern (ibid. Chap. VI, the description made by Darwin of the possible transformations is what are called affine transformations). Therefore, he writes, for each body plan (about 4 of them), of an "archetypic animal". But, if such is the case, there must exist one simplest description, among all instances of animal development, which encapsulates qualitatively all features of the archetype. The mathematical description of such an archetype, which will be given in the end of this review is a qualitative description of the other instances.

While I present in this review a mathematical description which may be called a personal point of view, the description of embryo movements in terms of a hyperbolic

mates, have quite different early stages of development. From a physicist's point of view, this is not accurate, and even misleading. It is clear that, apart from minor issues which do not affect the topology of the problem, these developments are actually very much similar if not identical. Two apparently important, but minor differences are, first, the fact that chicken embryos form from a flat disk, while frogs or mammals form from a round mass of cells. But in fact, these animals all form from a central part of that mass of cell, which departs only slightly from flatness, and although some embryos may be more curved than others at start, due to the underlying geometry, the topology of the problem is the same. Second, many species grow by digesting the protein rich content of the egg, therefore growing significantly as they develop (e.g. chicken embryos digesting the yolk), some others, like the frogs, have less vitellus, and will form at roughly constant volume, the cells getting smaller and smaller as they divide. Again, the topology of the problem is not affected by this issue, first because dilation effects are second order effects as compared to morphogenesis (as well known from simple inspection of child growth), and second because the truly morphogenetic events are quite rapid (of the order of a day).

5 "Archetype" is even an entry in the glossary of the second edition of Darwin's opus.

6 And plants plans may be "even fewer" than 5. (ibid. Chap. XIV). 
flow has been reached independently by others, especially, to my knowledge, by Cornelis Weijer of the University of Dundee, and his co-workers. Although there may be a debate over the exact role of advective ${ }^{7}$ transport vs. chemotactism, there is a general agreement about the existence of a hyperbolic (saddle) point.

The novelty, as regards the ideas of Darwin, is twofold: first the archetype plan corresponds to a simple physical symmetry breaking occurring in the ovocyte which is scaled "upwards" to the final animal shape by a hydrodynamic ("emergent"), flow field, and, second, the actual animals are obtained from the archetype by multiple extensions along one dimensional growth fields, such as digital rays, which are fixed by the anisotropies of physical fields. By following blindly the fantastically advanced intuition that animal bones could more easily be stretched or flattened (affine transformations), than bent or twisted, Charles Darwin leapfrogged the deep physical question of why should development be so much constrained. In effect, Charles Darwin in the same opus, states at several places that the possibilities of morphological adaptation "have no limits", which is contradictory with the observed restricted set of affine skeletal extensions. However, such affine transformations occur only after the hyperbolic flow has established the global pattern.

The novelty as regards D'Arcy Thomson is that D'Arcy considered mostly these affine transformations, say between one fish specie, and another one, and did not propose a deformation field for the appearance of the archetype itself; however, there is no way an affine transformation can transform a round blastula into anything resembling a fish, or a mouse. The transformation that forms an archetypic fish, from a round blastula is a vortex flow, with a hyperbolic singularity, as has been demonstrated recently.

\section{The invertebrate morphogenesis.}

\subsection{The fly bauplan ${ }^{8}$}

The initial "formless" animal is either round, as in vertebrates, or oblate (cigar-like), as in the fruit fly (and also in C. Elegans). This to say that the fly ovocyte (syncitium), is roughly a one dimensional tube. In the fly ovocyte, early gradients of maternal proteins exist (bicoid, nanos) which set an antero-posterior ${ }^{9}$ gradient, however it is not clear

\footnotetext{
7 Transport by convection, without active feedback forces.

8 A long tradition of german embryology has established the word "bauplan" or "blueprint" for the body plans. For some, this word is dangerous because it gives the impression of an architect predetermining body shapes. However, if global animal patterns (such as bilateral, radiate, etc.) are determined by general principles of symmetry, then the word "bauplan" can be used safely.

9 Axis in the embryos are defined as antero-posterior (from head to tail), dorso-ventral (from back to belly), left-right, and proximo-distal (from near the body and extending away from it). Rostro-caudal is also used instead of antero-posterior.
}
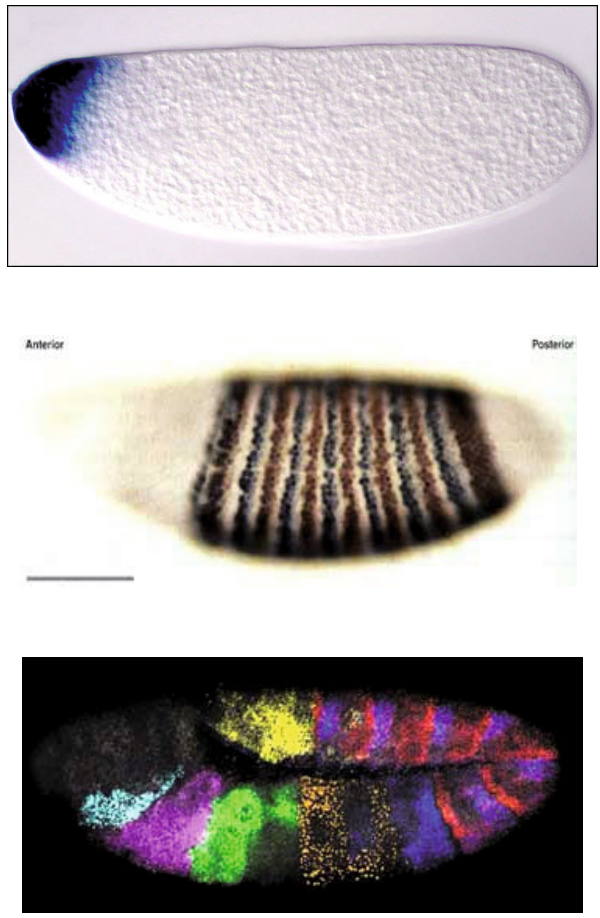

Fig. 3. (Color online) Three consecutive, very early, stages of the fly development. The images, from top to bottom show progressive expression of "morphogenetic" genes which establish the prepattern of the fly. Top: early gradient of maternal proteins (bicoid gradient, Irionn and St Johnson, Nature 2007) Middle: expression of Pair-rule genes induced by the early maternal genes (not shown even-skipped and frizzled genes also expressed at that stage). Bottom Hox genes induced shortly afterwards. At this stage, complex surface movements start. Description of techniques that generated the bottom picture can be found at McGinnis Lab: http://supefly.ucsd.edu/ labs/mcginnis.

how this gradient is actually "read" [19]. The fact that this gradient is a simple 1D monotonous decrease may be ascribed both to the fact that the high concentration source of (say) bicoid is positioned at one end, and to the fact that the ovocyte is quite elongated. It is known that, in the fly, these early maternal gradients are related to the formation of regular stripes of expression. Let us note that the gradient of bicoid is not found in all insects, and that some other gene has to take over this role in other species [20]. Specific genes, gap genes (there exist 4 such gap genes) "switch on" and interact along the body, in response to given levels of the said molecular gradient (Fig. 3), forming regular stripes which pre-pattern the embryo of fly at the syncitium stage (when it is formed of a single large cigar-like bag). Next, pair rule genes are expressed in alternating stripes. Gap and pair rule genes interact with each other in complex genetic networks which are still debated [21].

These chemical inductions do not rule out a role for geometrical constraints and physical forces: for example, left and right appendages are indeed totally different (mirror symmetry), and this may be tracked back to the mere fact 

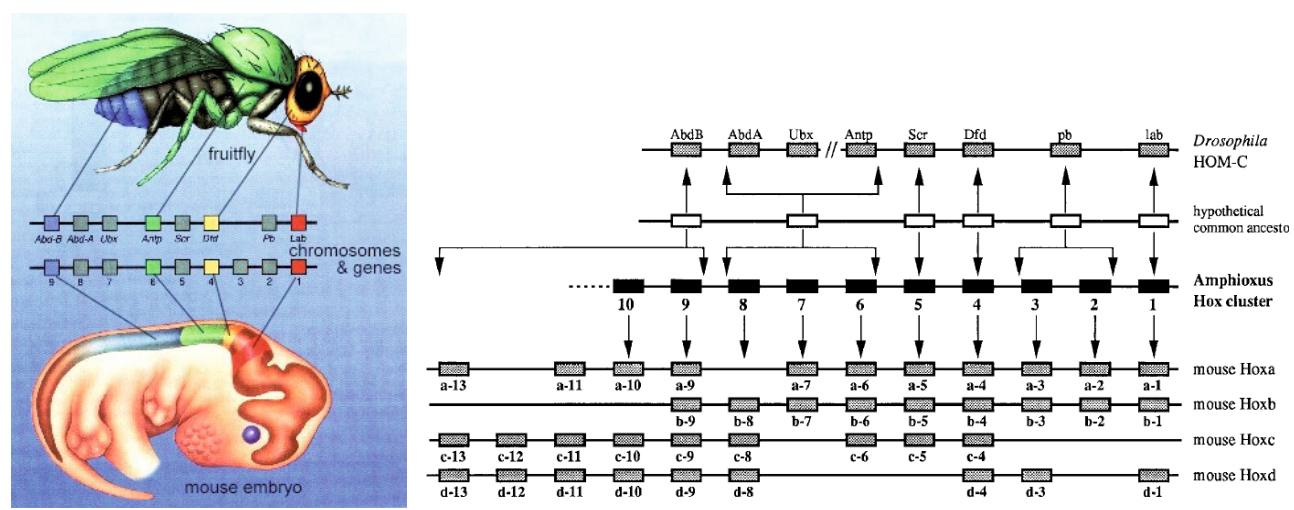

Fig. 4. (Color online) How "morphogenetic genes" are distributed along the body axis. Left: collinearity of genes along the genome (inside each cell) and their spatial expression inside the multicellular tissue. From Arizona University Biology Learning Center, Educational course 1. Right, genetic homology between an arthropod (fly), a primitive chordate (Amphioxus), and a mammal (mouse), showing the homologies, and inferred genetic duplications which occurred during evolution. From these analyses it is inferred that there must have existed a common ancestor with a more restricted set of such genes. (from GarciaFernandez, Holland, Nature 370, 563 (1994)).

that the curvature of the fly egg is opposite on each side. In the same spirit, inside each stripe, cells are seen to align by local rearrangements. The stripes of expression correspond to actual cellular lines, which are congruent with concentration level lines. Current genetic networks do not take into account this cellular reorganization, likely linked to compression forces. Gap and pair-rule genes induce by genetic reactions, expression of homeobox genes, which "pattern" the animal, during complex gastrulation motions (germ band extension) which form furrows along the body (visible in Fig. 3, bottom), appendages form from cellular discs which nucleate along the sides of the embryonic fly at boundaries between segments.

\subsection{The homeobox genes}

Genetic analysis of the fruit fly, and its mutants, has shown that a number of genes (homeobox genes) are expressed from head-to-tail, in an order which is collinear inside the insect body, with the order along the genome. In the fly, these genes form a single cluster of 8 genes located on the same chromosome, and "collinearity" means that genes are expressed one after the other in space, in the same order which they have along the genome (Fig. 4). This means physically that there is a spatial arrow at the scale of an organ or body part, which is somehow converted into a spatial arrow at the scale of the DNA, and/or vice-versa. It is assumed that levels of chemicals, in gradients, induce the next genetic expression by threshold/network of reactions [22], or that timely inductions along the genome are transformed into spatial cues by growth; however, it has also been shown that mechanical forces are able to induce morphogenetic events in the fly [23], therefore, a mechanical gradient such as a static gradient of pressure or of tension may as well explain the spatial expression of given genes, which would be associated, for example, to mechano-sensitive proteins, like ionic channels [24]. There exists a model of collinearity based on the pulling action of cells upon Hox clusters which is claimed to recover most features of collinearity experimentally observed [25].

The "morphogenetic" genes are called homeobox genes, because they contain a domain (a "box") which codes for a protein which binds to DNA. This domain is a $183 \mathrm{bp}$ DNA sequence that yields a 61 amino acid protein [26]. Therefore, these genes code for proteins ${ }^{10}$ which interact with other genes downstream along chains of genetic inductions. Each gene is expressed sequentially in turn along the body axis of the fly, overlapping the previous gene expressions present in previous stripe. Therefore the body plan instructions are spatially segmented, and different biochemical pathways will be expressed in each stripe, downstream of the homeobox genes, expressed in an increasing number from head to tail. These pathways will "induce" either legs, antennas, halteres, or wings along the body by nucleation of appendage primordia at intersections of genetic boundaries. However, wings and halteres are more dorsal (hence the wings "on the back"), while antennas and legs are more ventral (hence legs hanging below the abdomen). Antennas can be genetically "upgraded" into legs, and halteres into wings, but more ventral appendages like legs cannot be morphed into more dorsal appendages, because the difference is not only a difference of genetic expressions: there is also a topological difference (what matters is both the nature of the proteins, and the spatial organization of the primordia).

Since gene expressions overlap in subsequent segments, the segment forms are obtained one after the other by adding new genetic expressions in a region physically downstream to the ones in the previous stripes. A simple proof of that, relies in mutations which are able to modify in a deterministic and spectacular way the animal: the mutation Antennapedia [27] is able to generate a fly with legs instead of antennas. Ultrabithoraxs [28] is a repressor of wing formation, in the next segment after

10 Called transcription factors. 
the normal wing segment. If inhibited or knocked out, it induces a fly with an additional pair of wings in the segment next to the normal segment that carries wings (in the place of halteres), etc. Therefore, the animal morphogenesis in this case goes this way: a chemical gradient inherited from the mother descends monotonously inside a cigar-like pattern at a very early stage; genes respond with thresholds/networks to this gradient, such that the cigar is segmented regularly, inside each segment different cascades of events occurs, due to different chemical properties. Physical deformations are observed essentially when the stripes are well formed, and areas of the segments arranged in rings, called "imaginal discs", extend outwards in the form of appendages ${ }^{11}[29,30]$ by telescoping out the rings. The appendages morphogenesis is called eversion. It consists in pushing out a form actually latent inside the annular structure of the imaginal discs. The actual shape and length of these appendages will depend on intensity of growth forces, mechanical properties, and other possible "inductive" cascades. The number of genes implicated in this phenomenon is large [32], but three genes are more important than others: Engrailed (En), Wingless (wg) and Decapentaplegic (Dpp). The appendage primordium is a set of approximately 20 cells localized at the intersection of the genetic boundaries of these three genes, which are associated to a localized singularity of growth. The lateral locations of these primordia along the thoracic and abdominal segments of the fly imply subtle geometrical symmetry breaking which induces the left-right and antero-posterior asymmetry of the limbs, and the differences between legs and wings (see a thorough review in Ref. [32]). Around the singularity, the 20 cells develop to form the concentric ring pattern, which later stretches outwards to become a true appendage.

Despite apparent differences, legs, antennas or wings are not so much different, and small genetic (biochemical) variations occur inside each body segment, which suffice to generate a great variety of insects. This to say that a basic, again, "archetypic" appendage is formed of tubular segments with decreasing diameters, as measured from the body and outwards, which are obtained by stretching out the annular domains of the imaginal discs. Looked in detail, legs appear as stretched antennas (Fig. 5), and so do wings with respect to halteres. While the initial geometry is the same, even genetic domains are very much similar (see Ref. [33], p. 583: "the halteres appear to be patterned in the same way as the wing".).

Considerable efforts are dedicated to finding the detailed genetic gradients which are responsible for the more or less stretched aspect of appendages, however this line of work always assumes a permanent appendage plan formed of telescopic rings [30]. It should be remembered that, from the point of view of morphogenesis, differences of a few per cent in mechanical parameters, may generate

\footnotetext{
11 Please note that the initial pattern of morphogenes is oriented from head to tail, while appendages outgrow laterally from the segments. This lateral outgrowth might be due to local tangential stress which induces perpendicular outgrowth (buckling force).
}

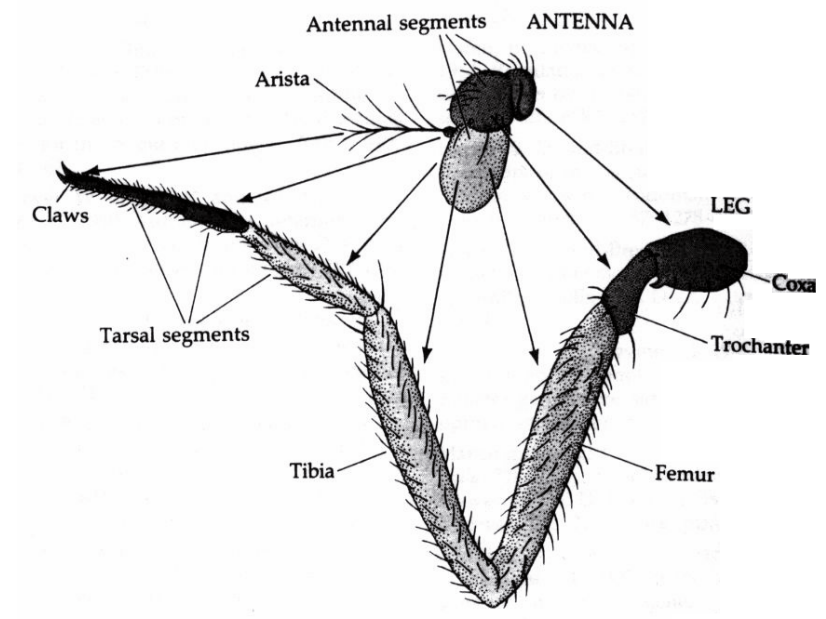

Fig. 5. Classical anatomic description of legs and antennas. Actually, all parts of these appendages are homologous, and legs appear merely (at least morphologically) as stretched antennas (from Genetics of axis specification in Drosophila, p. 570, after Ref. [31]).

such different shapes as elongated viscous fingers, or fractal patterns [34-36]. In the case of structures with radial symmetry, with radial or ortho-radial fiber order, it has been shown theoretically that they tend to grow outwards in the direction of the axis of symmetry $[37,38]$, which provides an anisotropy of the surface tension, or of the surface bending modulus, therefore, there is very little doubt that appendage growth is stabilized by its chemical structure, much as plant stems grow in directions parallel to wood fiber. This is because free boundary problems in which such physical quantities as surface tension, bending modulus or viscosity, etc. play a driving role, tend to stabilize exponentially fast the anisotropic solutions [36].

\section{The genetics of vertebrate development}

\subsection{The homeobox genes in vertebrates}

Although this explanation is terse, it is important to know the situation in insects, in order to understand the intellectual background, and how much vertebrate development differs from insects. In vertebrates, genes homolog to genes of insects have been found [39], especially homeobox genes, which raised the optimistic hope that vertebrate development was organized just as insect development. These genes are again present in rows ("clusters") of genes physically aligned along the DNA, although such clusters may be found on different chromosomes [40]. In mammals, there are four clusters named Hoxa, Hoxb, Hoxc, Hoxd corresponding to up to 13 groups of genes, instead of the unique cluster of 8 genes of the fly. Genes of different clusters are often expressed spatially simultaneously, and corresponding genes in different clusters may have redundant activities, the deletion of one gene being rescued by another. The increase of Hox genes numbers and clusters is 
due to genetic duplications during evolution. This renders the notion of collinearity "more complex" in vertebrates. However, it should be noted that the general bauplan of vertebrates is universal (head, body tail, and limbs sideways), despite considerable variability of gene clusters and gene counts, between say, fish, and humans. There seems to be something deeper in the global plan.

The Hox genes certainly play an important role in vertebrate development, since knock-out experiments (which consist in generating mutants which lack a given gene), show morphological modifications ("phenotypes"). Rules for the functioning of these genes have been established such that: they are expressed in collinear order along the trunk, and more genes are expressed in the more posterior regions. For example, mice having shorter body and longer tail may be generated by affecting the gene Hoxa2 [41]. In the same spirit, a modification of the gene Hoxd3 induces an anatomical modification of vertebrae in the region of the neck [42]. But it is clear that "homeotic" transformations in vertebrates are much more modest than in flies [43]. Homeotic transformations are body form changes due to deletion or misexpression of Hox genes. There does not exist mutants having hands instead of jaws, or feet instead of hands, or skulls instead of phalanges. There seems to be something qualitatively different between the segmentation process in flies, and in vertebrates, which can be tracked back to the fact that arthropods segment very early, such that downstream genetic pathways are more localized than in vertebrates. In vertebrates, segmentation occurs during the early morphogenetic movements which form the body plan, but somewhat after the start of the motion, during folding. The segmentation in vertebrates is a uniform instability wave which propagates in an already formed embryo. Hence a vertebrate can be described as "an animal body with segments inside" (the bones), and not as "a segmented animal" like arthropods. Hox genes modulate the identity of segments, they do no create them.

In vertebrates, at most, modest shifts of body parts, or growth of extra ribs can be "homeotically" induced by change in Hox genes. These shifts seem to follow some existing stream or path. For example: it is possible to shift the beginning of the tail, but more anterior or more posterior to the tail direction, not to the neck, or to the wrist, it is possible to shift the limb position by one vertebra along the thorax, but not to the forehead, it is possible to generate a mouse with longer or shorter limbs, not with an ectopic skull in the hand region, etc. Generally, homeotic transformations tend to shift the form, and produce "posteriorisation" or "anteriorisation" of forms. This is due to the collinearity of the genes expression with spatial order. Why this is so will become clear below.

However, it is no at all true that there is, in vertebrates, such a strict collinearity between the genetic organization in the nuclear DNA, and the organization of the body plan. Limbs form by growth of limb buds away from the body axis. In the case of insects, the body axis is quite well defined, and so is the notion of "lateral outgrowth", or of "away" from the body axis. In the case of vertebrates, there exists rotational engulfments of the tissue (as we shall see in Sect. 5 , such that the body axis is not a relevant concept in areas of strong bending or twist.

Moreover, by definition, DNA sequences are linear, and if collinearity between DNA sequences and body form exists as a concept, then nothing can molecularly specify lateral "branchings", or outgrowths. Lateral outgrowths require a change in growth direction. But such a change is not present as a specific instruction in the genome. This is to say that, while the subsequent genes in the Hox clusters may be correlated to specific forms observed in the stripes (where such or such genes are expressed), there is no interruption of the cluster of genes, by any gene which would serve to instruct a right turn to the growth process. The symmetry breaking which orients growth sideways is a physical, implicit one ${ }^{12}$, this is to say that it is an instability of the growth process itself, an anisotropy or a tensorial feature.

In addition, it is known that genetic expressions in limb buds make use of similar genes in the hindlimb, and forelimb, and in the tail, namely genes Hoxa, Hoxd 9 to 13 are used for all these 3 body appendages of vertebrates [44], in a proximo-distal order.

Therefore, while it is indeed true that vertebrate limbs are "some sort of a tail" growing sideways, the points of outgrowth of the limbs do not correspond to a sequential order of genes, in specific segments or boundary intersections that would induce specific appendages, as in flies ${ }^{13}$. The tail is not located at a specific segment, nor are the limbs, the ears, the jaw or whatever. It may be possible that collinear sequences of homeobox genes are re-used obliquely along the body in order to generate the limbs, after having been used along the body to generate headto-tail pattern, but this concept does not explain why it is so. Moreover, recent data show that the distal part of the limb expresses Hox genes actually in a reverse collinearity pattern [45]. Detailed analysis of mutants shows that "Unlike the situation in the trunk, and in contrast to what was originally expected, loss-of-function phenotypes cannot be interpreted as classical homeotic transformations." (Zakany and Duboule [44]). These authors conclude that the outcome of Hox genes function is "non specific", "flexible", and related to "context". This is to say that boundary conditions, and previous history will be a concern for the actual outcome.

If genes are induced sequentially by automatic inductions, linked possibly to gradients of some triggering molecule, the existence of reverse orders of expression imply a complex spatio-temporal organization of the genetic expressions. The collinearity observed along the trunk might be an erroneous concept, which appeared important only because the physical object "trunk" is almost 1 dimensional. It is important to note that the concept of collinearity requires a $1 \mathrm{D}$ physical substrate, but the origin of the 1 dimensionality is completely different in an insect

\footnotetext{
12 As is, also, the point of intersection of the domains of En, Wg, and Dpp from which insect appendages emanate.

13 Also, while the appendage primordium in insects is a very localized cluster of approx. 20 cells, in vertebrates, the limb bud encompasses thousands of cells.
} 

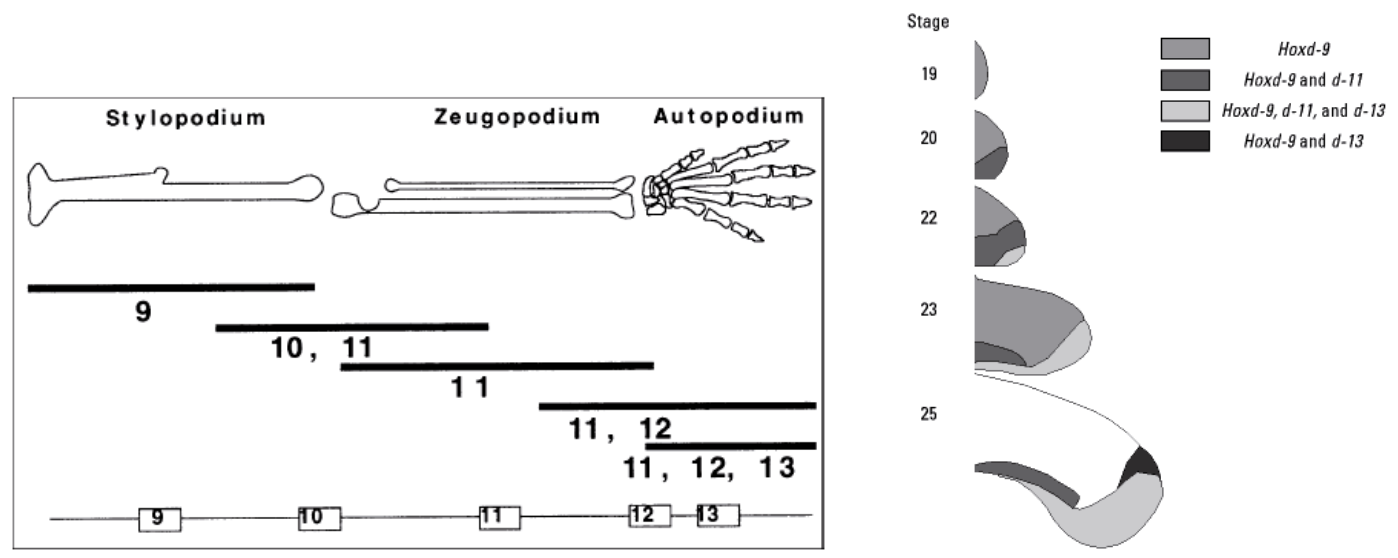

Fig. 6. Left, genetic collinearity between clusters of genes and limb development, as deduced from knock-out mutants (from Ref. [60]). Right, more detailed 2D genetic expression in the limb bud. As the limb extends, the gene expressions forms specific areas, although they are not strictly correlated to hand patterns. As time passes by these domain of expressions become more fuzzy.

(elongated syncitium) and in a vertebrate (formation of median folds, see Sects. 6 and 7, especially Fig. 40). At present, the formation of a vertebrate body is grossly described by a collinear expression of Hox genes from head to tail, a collinear expression of genes from shoulder to wrist, and a reversed collinear expression of genes in the autopod ("the hand"). However, how all this transforms into morphogenetic fields which generate bone, tendons, joints, etc. is not clearly established, but assumed to be largely related to early embryo movements [46] (Burke and Nowicki state that: "It seems likely that the establishment of Hox expression patterns, and the positioning of cell populations are related, thus insuring harmony between anterior-posterior and medial-lateral patterning axes" ).

\subsection{Limb patterning in tetrapods}

In order to attempt to bring vertebrate development towards the description of insect development, a number of genes have been pointed out whose role seems somewhat analogue to what is observed in insects. The position of limb outgrowth has been correlated to the levels of Hoxc6, Hoc8, Hoxb5, Hoxb6, Hoxb9, Hoxc9, Hoxd9 genes [44,47]. It appears that hindlimb and forelimb seem to protrude out at boundaries of expression of Hoxc6 gene, or Hoxc8 gene. It has also been shown that snakes express Hoxc6 gene in a much more expanded area as compared to other tetrapods (Fig. 7), thus correlating to the position of vestigial limbs in these animals [48]. Also, homeobox genes have been related to the presence or absence of ribs in relation to vertebrae (which suggests that actually, insect appendages are homologues of ribs, and not of vertebrate limbs, which are something else). However, as stated by Rallis et al. "neither gene deletion nor gene misexpression experiments have provided direct evidence for a role of Hox genes in limb positioning" [49]. Limb positions seem to be related to the thoraco-sacral junction, and to the thoraco-cervical junction, in all animals. Therefore, the outgrowth of limbs seem geometrically linked to these

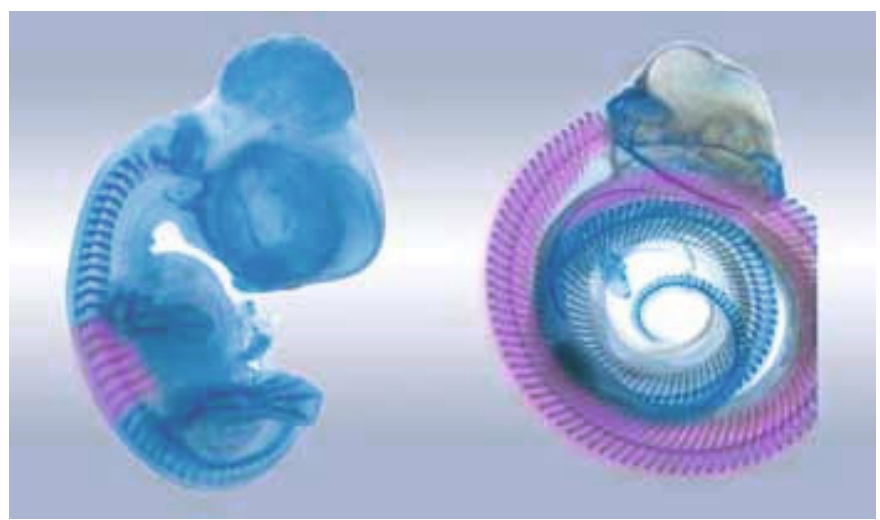

Fig. 7. (Color online) Domain of expression of Hoxc6, in a chicken, left, and in a snake, right. The evolution from tetrapods to snakes seem to imply an extension of the domain of Hoxc6, rostrally, and caudally. Photo by McOmber and Burke.

anatomical regions, by the morphogenetic process, and the Hox genes domains of expression may be more a consequence, than a cause of limb positioning. From a physics point of view, it seems that limbs do not form "at" the thoraco-sacral and "at" the thoraco-cervical junction, by some molecular coincidence, but that these junctions come together with the limbs in the morphogenetic process. The pelvis, for example has a large and complex shape spanning many vertebrae; it shows a clear vortex pattern both in the global shape and locally in the trabecular bone orientation. It is not a shape with a defined "axial" form. In addition, it has a concavity in the area of the leg joint associated to antagonist growth of the cartilage cups of the pelvis and of the femur (please refer to a general anatomy textbook). Clearly the pelvis shape is linked to the entire, extended, physical field of morphogenesis, and not to a simple inductive axial ("collinear") event that would "trigger a pelvis" in a definite, strictly instructed, shape. It is extremely unlikely that a "pelvis" could be induced 


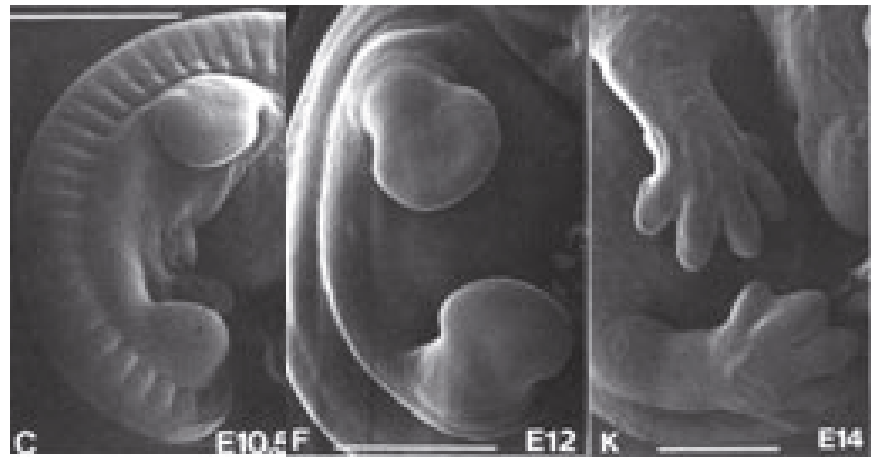

Fig. 8. Direct observation of limb development. Formation of the limbs starts by a lateral plate (limb field) out of which little paddles extend, to become progressively true limbs. At the moment of formation of the limb paddle, the segmentation wave has descended along the body backbone, generating the presumptive vertebrae. The wavelength of these segments seem to influence the wavelength of segments in the hand (digital rays, which become true fingers after cellular death in between finger bones), because the number of somites facing the limb bud is always close to the finger count. A direct count of the segments shows here that there are indeed about 5 segments in front of the paddle, before it extends. From Martin, University College, London, UK.

anywhere else by a genetic induction. Also, in many animals the curve of the pelvis continues itself in the shoulder girdle; the hindlimb grows out at a specific node of the pelvis, and not truly "along the trunk", etc. If the concept of genetic induction were true, one would expect to see mutants with a pelvis without limbs at any given level of the thorax, and, anywhere else, limbs without pelvis or shoulders, which is certainly not the case.

Whatsoever, these facts do not fit into an anteroposterior gradient of anything, especially since the domains of expression of Hoxc6 shift both towards the head, and towards the tail, when going from tetrapods to snakes. Therefore in vertebrates the concept of antero-posterior gradient is not related to the correct positioning of limbs.

The further patterning of the limbs remain largely mysterious. It has long been known that limbs emanate from "lateral" plates of the embryo, which constitute a "limb field". The limbs grow outwards in the shape of paddles, and eventually limbs (Fig. 8).

There exists a considerable literature dedicated to the morphological commitment of limb buds, as a function of grafts, ectopic ${ }^{14}$ expressions of molecules, genetic knock outs ${ }^{15}$ or mutants, etc. Molecular studies distinguish the role of transcription factors, which bind to DNA (homeodomain genes), and of signalling molecules, such

\footnotetext{
14 Ectopic grafts or expressions, consist in positioning a graft or a molecular source in an aberrant place, and watch the developmental outcome. For example, ectopic limbs can be obtained in aberrant places, by putting ectopically a bead of some chemical under the ectoderm, in that aberrant place.

15 To knock out genes consist in generating an animal in which all alleles of a gene are absent. A mouse ko for a given gene will show traits related to the absence of that gene.
}

as growth factors, which maintain proliferation, etc., but without affecting the DNA (Fig. 9).

It is especially clear that fibroblasts growth factors ${ }^{16}$ (FGFs) produced by mesodermal cells are involved in limb outgrowth expansion, since implants of beads soaked in FGF1, FGF2, FGF4 or other chemicals generate ectopic (supernumerary) limbs [51], and mutants lacking FGF10 have no limbs (in addition to having no lungs [52], but it should be noted that they do have pelvis and scapula rudiments). Also, double deletion of FGF4 and FGF8 annihilates limb growth, in addition to many other abnormalities [53]. Annihilating FGF8 expression only, has no effect on limb pattern, while double deletion of FGF4 and FGF8, impairs limb growth, but does not prevent the formation of the initial bud, which is positioned at the correct place. This shows that the relationship between growth factors and the final pattern is somewhat puzzling, there seems to be strong "redundancies", and "regulatory loops", although the rationale may be otherwise: as long as quantitative levels of growth factors are not related to physical parameters of growth, it is hard to tell why a limb grows or not. It seems that the topology of the pattern is actually not related to the growth factor: either the pattern grows (because cells divide actively), or it does not, but if it does, it grows with a topology independent of the growth factor, which would be coherent with the idea that growth factors are not morphogenes, like Hox genes. Nevertheless, cellular proliferation induced by FGF growth factors seems the crucial developmental engine of the buds, to form limbs, this is why FGF's are considered as the "controllers" of the proximo-distal axis of growth.

Now what starts this proliferation, and why there, and how is the limb type determined? Genetic studies seem to imply that it is the role of transcription factors such as the Hox genes to modulate the magnitude of these growth factor along the flanks, by the dosing or timing of many molecules downstream. Considering the pattern of expression of Hox genes [47,48], and loss-of-function phenotypes [44] it is unlikely that Hox genes provide a spatial code or a mosaic for the limb pattern, but rather subtle quantitative gradients of parameters of some physical process of patterning.

The exact positioning of the limb, and limb type has been related to the transcription repressors of the Tbox family $[54,55]$. It has been shown that it is possible to shift slightly the position of outgrowth of the limb by repressing or activating Tbx3 [49], but shifting a limb is not the same thing as inducing a limb ${ }^{17}$. Negative misexpression

\footnotetext{
16 Fibroblast growth factors are molecules which (among other roles) maintain the proliferative activity of fibroblasts. There exist about 23 members of this family, with similar sequence. There exist 4 receptors of these growth factors. Fibroblasts are one of the major cell types. They provide the production of collagen and related supporting molecules. Hence FGFs and their receptors play a key role in many aspects of embryo development, angiogenesis, etc.

17 When limb shift is obtained in a biochemical assays, it is generally accompanied by a strong flexion of the embryo body. Experiments in which the contralateral side is not modified
} 

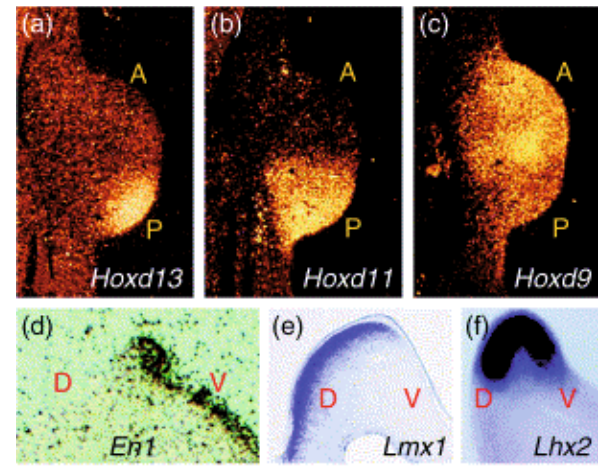

(e)
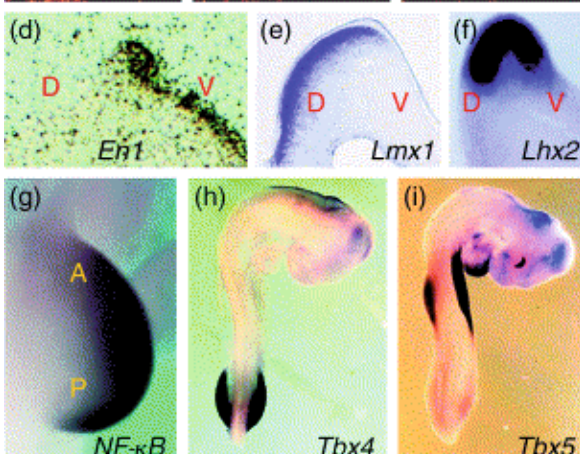

Tbx4

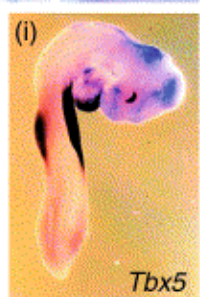

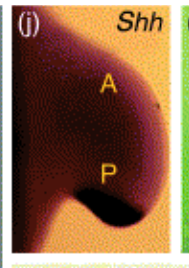

(I)
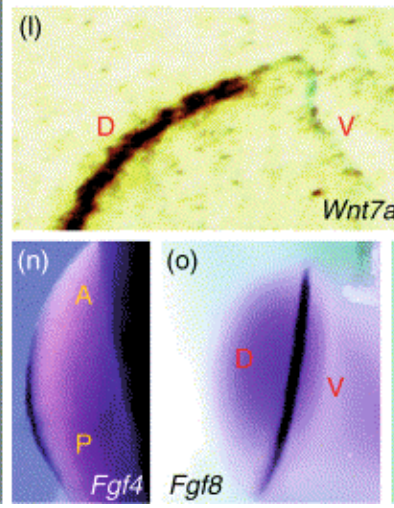

(0)

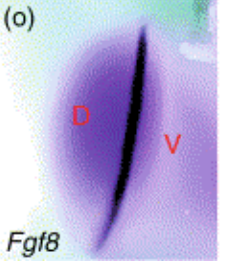

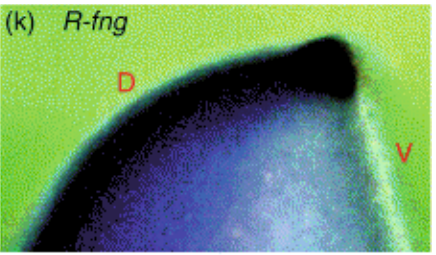

(m)

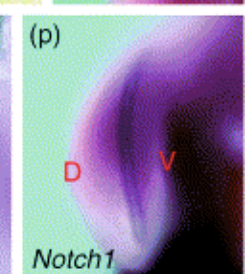

Fig. 9. (Color online) Stainings of several molecules in the early chick embryo. Left transcription factors, right signalling molecules. (From Ref. [49], and references therein). Please note the strong Tbx4 and Tbx5 stainings in resp. hindlimb, and forelimb. The Tbx4 staining is clearly linked to the mesoderm engulfment, and not to some linear gradient: the crescent shape of the deep blue Tbx4 staining follows the rotational pattern of cell flows in this area (more in Sects. 5-7). Color stainings should be taken with some care, as the stainings follow actual twists of the tissue.

of $T b x 5$ [56] leads to a massive reduction of lateral plate in the forelimb area, and complete absence of the forelimb in the newborn, Figure 10. But still, there is a lateral plate.

The origin of identity of the limbs remain mysterious: early hopes [50] suggested that limb type was related to the pattern of $T b x 4$ and $T b x 5$ genes, since these were specifically present in resp. hindlimb, and forelimb of chicken (see Fig. 9h,9i). Also Pitx1 seems to be specific of hindlimb, and may play a role in hindlimb identity vs. forelimb. Loss of function of Tbx5 in the forelimb leads to complete absence of forelimb as shown in Figure 10. When expressed in place of $T b x 5, T b x 4$ was able to rescue the formation of the forelimb [57]. But surprisingly, the limb was of a normal forelimb type. Unfortunately $T b x 5$ and $T b x_{4}$ were found in limbs of both types altogether in the newt model [58], which sheds doubt on the relationship between $T b x$ and limb identity. Electroporation experiments, by which $T b x 5$ is expressed in legs are somewhat controversial: the leg has only 3 digits, like a wing, but the phalange count is not correct ("misexpression of Tbx5 in the leg can induce wing-like skeletal patterns, albeit partially " Takeuchi et al. [59]). We all know that chicken legs carry scales on the skin of anckles and digits, while chicken wings carry feathers: scale to feathers conversion on the legs can indeed be obtained genetically [58]. The wing in which $T b x_{4}$ is expressed (while $T b x 5$ is the normal Tbx gene there) looks qualitatively like a leg: the fingers of the wing separate, as in a normal leg, and the fingers have claws. The success rate of these experiments

and serves as control shows that shift of limb position comes together with a global deformation of the entire embryo side, generally more elongated in the direction of the shift $[46,51]$. is very low $(\sim 10 \%)$, and puzzling for physicists. Especially one would expect electroporation experiments of $T b x 5$ in a right leg bud to give either $50 \%$ of left wings and $50 \%$ of right wings, if limb identity is only defined by $T b x 5$, or $100 \%$ of right wings, if the limb identity is defined by Tbx5 and the context. Still $90 \%$ of right legs are actually obtained $^{18}$.

While there is indeed an impact on skeletal elements of the normal limbs of misexpression of genes, the extra limbs formed by expressing ectopically $T b x 5$ or $T b x 4$ in a wrong area are generally not normal. Again, there seems to be "something else" in the spatio-temporal outgrowth of limbs than just the expression of transcription factors, and artefacts are not excluded. Especially, while the presence of $T b x 5$ is necessary for limb outgrowth, what determines the localization of $T b x 5$ and the gradients that create the bias in limb axis?

In the same spirit, the identity of the ectopic limbs generated with soaked beads is not clearly related to the growth factor inserted ectopically, but more likely to the embedding tissue (the posterior or anterior nature of the limb depending on the position along the flanks), and the way it is disrupted by the insertion of the bead (especially in terms of symmetry breaking of the bud orientation; insertion of beads leads to complex digits distributions). It is rarely clear what exactly ectopic expressions of signalling molecules prove about the limbs in the normal case: in the normal case there are no beads, and it is proven that several molecules used historically to induce

\footnotetext{
18 According to the authors, Tbx5 is actually quite mechanosensitive (Ogura, Personnal communication, and in preparation).
} 

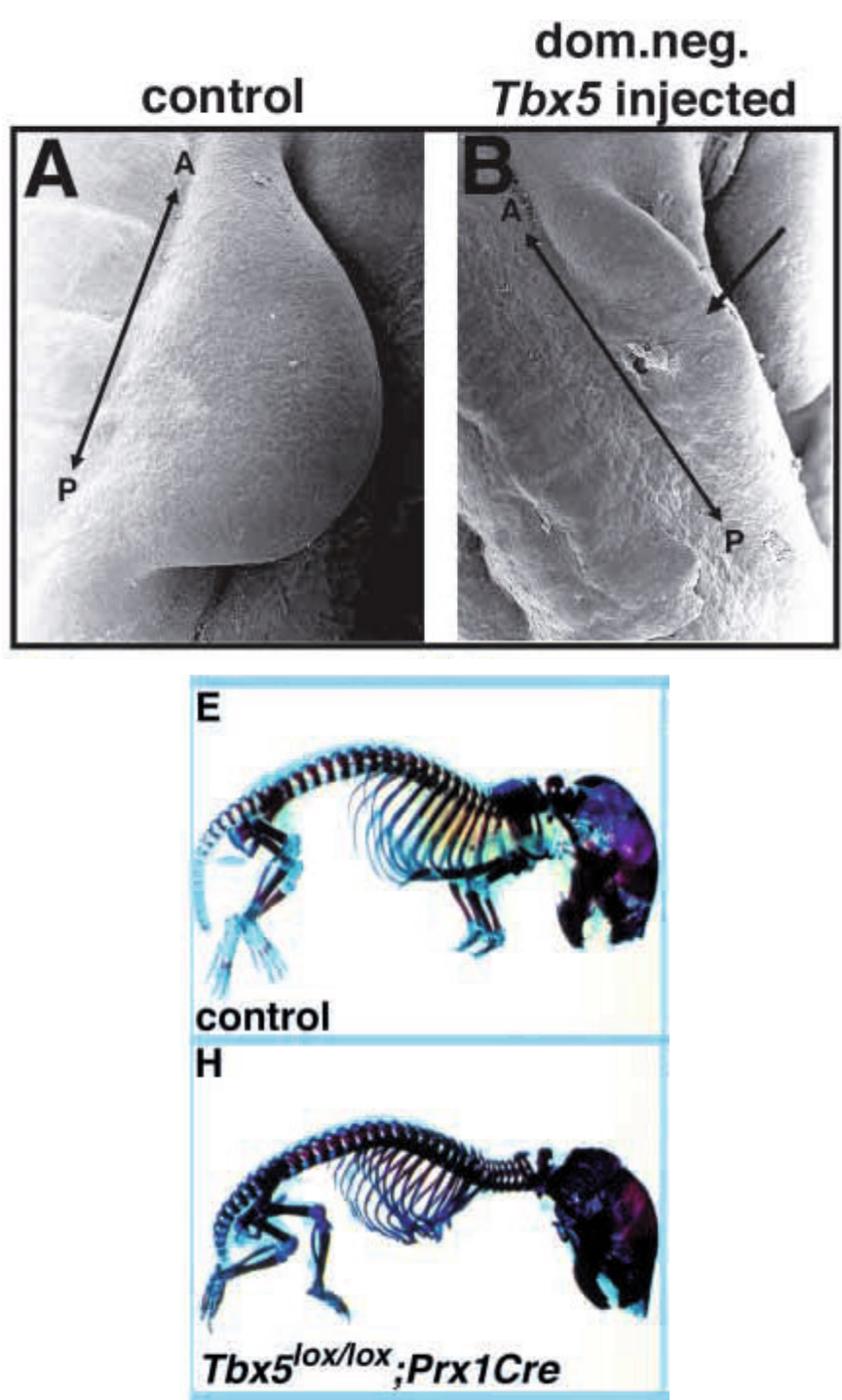

Fig. 10. (Color online) Typical genetic assays on limb development. A mutant is generated which lacks a given gene, here Tbx5. A massive reduction of the lateral plate (region of the presumptive limb) is obtained in the mutant mice defective for $T b x 5$. As a result, the mice have no forelimbs. From reference [56]. (By the way, one may remark that the number of somites in front of the early limb field is of the order of 5).

ectopic limbs [51], are just not candidates for normal limb outgrowth initiation.

The pattern of fingers itself has been related to molecular gradients in the limb paddle. Schematically, the limb paddle seems to be chemically organized in the following way. First, there exists a pool of a signalling molecule Sonic Hedgehog (SHH) [60-62] located at the frontier between the limb bud and the flank in an area called Zone of Polarizing Activity [62]. The gradient of SHH, and the time of exposure to $\mathrm{SHH}$ seems to be related to the identity of the observed fingers. Briefly stated, the gradient of SHH inside the hand, in the thumb-to-auricular direction seems to play an important role in the difference in shape of these fingers. But this is not true of all fingers. Nested expressions of Hoxd genes [63], and reverse collinear expressions of genes in the "hand" play also a role, but the overall regulation of these genes is not understood. While gradients of molecules may be deterministically correlated to finger patterns, how the molecules actually pattern the fingers is not understood. For example, placing a source of $\mathrm{SHH}$ opposite to the normal source of $\mathrm{SHH}$, mimicking a second zone of polarising activity, induces a forelimb with mirror distribution of fingers [64]. The activity in this field of developmental biology consists in finding the proximodistal $^{19}$, antero-posterior, and dorso-ventral gradients of molecules which would be responsible for the 3D spatial organization of the growth process (and their temporal expressions) SHH and hox genes are candidates for "controlling" the antero-posterior gradient, but a considerable number of other genes have been implicated, whose activity is related. Especially Hoxb8 seems to induce $S h h$ which induces Fgf8. Wnt and Fgf are related, Gli3 and Shh are related, Shh induces the cartilage forming pathway via Gremlin (Bone morphogenetic protein, BMP), etc. These molecules interact by positive and negative feedback loops which are rarely understood in detail.

Now, the proximo-distal axis of the paddle is said to be "controlled" by an area of the limb edge, known as apical ectodermal ridge (AER). This area has been shown for long to play an important role $[47,50,61]$ in order to maintain the proximo-distal arrow of growth. This area is a narrow thickening located along the distal edge of the limb paddle, which seems to feed the tissue in between the tip of the limb, and the flank of the required growth factors by an interplay of Fgf and Wnt genes, but again it is not clear whether these growth factors actually have a patterning role. The edge itself seems to be the line of suture of the endoderm and the ectoderm (see Fig. 1 in Ref. [49]), and the fingers form actually away from this thickening, more inwards the paddle, and towards the flanks.

Many experiments, most of which consisting in severing parts of the limbs, or transplanting parts, lead to long lists of results (mirror fingers, reduced distal elements, etc.), which remain controversial [65]. It is clear that the potential to form complete limbs or additional fingers is widespread along the body, with even tail cells being able to form digits if transplanted at the proper place, etc.

The apical ectodermal ridge, generally thought to be so important for limb development, was even found to be totally absent in some animals (the so-called direct frogs [66], which are frogs which form legs normally, without passing through the stage of a limbless tadpole).

Finger types are certainly related to the distal expression of Hox genes, but not their number. The formation of bony structures in the hand and limb seem more of a biomechanical wave of material instability (denser tissue forming the cartilaginous pattern of bones [67] by a process of self-enhanced attraction of fibroblasts), independent of the Hox genes, and Shh is an important parameter in this process. This is also true of vertebrae segments. This is apparent in the fact that many extent species,

\footnotetext{
19 Proximal $=$ close to the body, distal $=$ more away from the
} body. 
or mutants, have repeated sets of identical fingers $[68,69]$, sometimes largely exceeding the available number of Hox genes. Therefore there is something quite simple in the generation of rows of radiating digital rays, which span the limb paddle like an instability wave ${ }^{20}$, much like the formation of vertebrae along the spine [70]. Hox genes modulate the final shape of these domains of instability, but with a moderate impact. It seems that Hox genes come into play "too late", when the digital rays are already established. If $S h h$ is completely suppressed, limb buds still form fairly normally, but aberrant limbs extend, which may have for example a single finger [71]. The entire shh-/- animal (animal in which both maternal and paternal alleles of the gene $s h$ are knocked out) body is however completely aberrant.

Now, about the dorso-ventral "gradient". In general terms, the body exhibits a dorsal side and a ventral side, inherited from the existence of slightly different cellular types on the endoderm and the ectoderm. This dorsoventral polarity is also present in the hands, with an obvious palmar and a dorsal side. Bio-chemically, this difference seems to be related to the genetic pathway of en 1 and wnt- $7 a$ [62], and analogous genes may be at play in the dorso-ventral polarity of insect wings [33]. But the expression of specific genetic pathways is related actually to the geometry of the limb bud at the moment of formation: if the limb bud forms from endoderm and ectoderm ${ }^{21}$, it has dorso-ventral polarity (like a normal hand), if a limb bud forms more proximally by a fold of ectoderm only, the limb paddle has double dorsal polarity [62]. Therefore, the dorso-ventral polarity is a matter of gradient of molecules, but the boundary conditions for these molecules are geometrically carried by the respective layers of cells. From a geometrical point of view, the formation of the limb bud appears as a physical process, which does not require specific cell sheets, but it happens that ectoderm and endoderm have originally a molecular difference, and this suffices to induce a dorso-ventral "gradient" across the dorso-ventral direction.

The genetic descriptions of limb patterning get progressively more and more complex and controversial, and the view of how the vertebrate body is actually organized gets progressively more cloudy. The gradients of molecules forming an orthogonal system of coordinates do not seem to suffice to describe cartilage patterns inside a hand. Since such a representation is systematically used, I, too, have used it above to describe hand formation. However, when described in detail, the pattern of bones in the "wrist" of a mouse seems actually to have under-

\footnotetext{
20 The digital rays, regularly spanning the limb paddle, are called the "digital arch".

21 The initial formless embryo (the "blastula") is composed of one layer (the epiblast). A second layer, the hypoblast nucleates underneath the epiblast, therefore the hypoblast emanates from the epiblast. During morphogenesis, a third layer appears by invagination, during gastrulation, of the first two layers inside themselves. In the literature, as the body forms, new names are ascribed to the layers: ectoderm (skin on top), endoderm (layer below), and mesoderm (in between).
}

gone a flexion, or rotation, as compared to a fish $\mathrm{fin}^{22}$ (the sturgeon) [73], which cannot be described by orthogonal gradients.

Still, a primitive limb is such a simple thing: a round paddle with rays. A number of facts explain this darkness. First of all, genetic inductions always need a previous genetic induction, so that it is never clear what is the chain of inductive events to take into account, and this is all the more true as the movements which advect the patterns of expressions are not taken into account. The second fact is experimental: molecular experiments consist generally in expressing in an aberrant concentration, and in an aberrant place, a molecule normally present in the physiological case. It is therefore not at all clear how the experiment should be interpreted, if one does not know how the said molecule is produced in the physiological situation. For example, if it is quite simple to generate a supernumerary limb with a bead soaked in retinoic acid, does it mean that it would be very easy for nature to produce such a supernumerary limb? Or does it mean that it would be extremely difficult, because nature is unable to provide a soaked bead? Stated otherwise, what is the equivalent of the bead soaked in retinoic acid, in the physiological case? How does nature manage to create such a starting point for a limb? If localisation of any molecule, possibly FGF10 or Tbx5, is necessary to generate a limb, how is it localised, in the natural case, and why is the limb induction possible with so many molecules? More specifically, if the entire flanks between tail to shoulder is competent to give limbs, why do we have only four? If the word "genetic induction" has a meaning, why "induction" of a limb is not possible ahead of the shoulder and caudal to the pelvis, with the same "inductive" molecules that "induce" a limb in between shoulder and pelvis? [51].

Another problem lies in the spatio-temporal aspect of the development. It has been stated that "One of the most mysterious features of morphogenesis is that structures form themselves as they grow" [32]. In the same spirit, recent analyses in terms of gene regulatory networks have raised some surprise, since the same genes regulatory networks seem already present $570 \mathrm{My}$ ago in cnidarians (jelly fish, etc. [72]); therefore if the same genetic networks are present, either some minor molecular subtleties, downstream of the main genetic kernels, make the difference between animal bauplan (say hydra and rabbit), or it is a matter of spatio-temporal organization (different symmetry breaking) of the same genes with non-chemical boundary effects and wavelength locking.

Most, if not all, present molecular descriptions assume an existing area, for example a limb bud, or a body axis, and try to organize the chemical fields inside or along it. However, it is clear from a physical point of view, that body, organ, or appendage development is a free boundary

22 As will be discussed in Sections 5-7, the affine stretch of the bones (seen for example in the bats forelimb) is possible on digital rays which have a roughly 1-D morphotype. In areas where tissue rotations occur at early stages of development, the coupling between bone segmentation and tissue movements leads to less obvious bone configurations. 

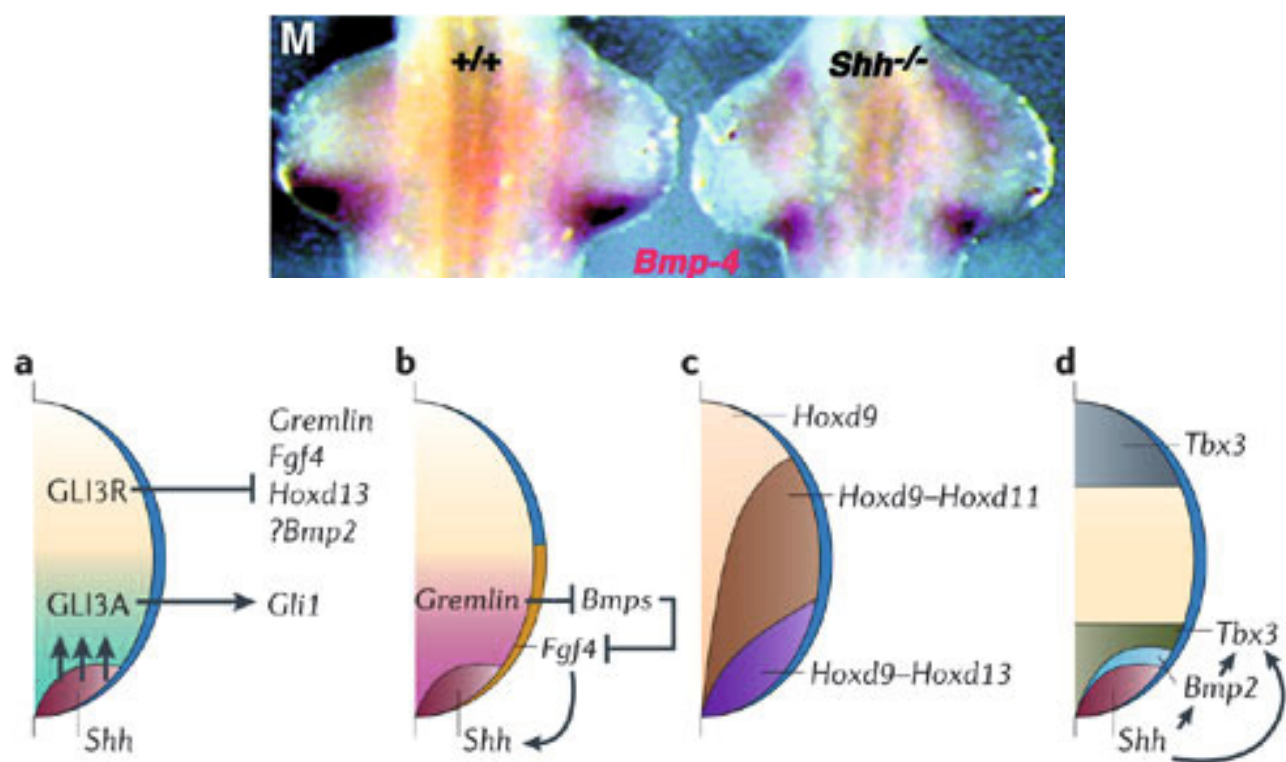

\section{Copyright (C) 2006 Nature Publishing Group Nature Reviews | Molecular Cell Biology}

Fig. 11. (Color online) Top (from Grotewold, Rüther, The EMBO Journal 21, 966 (2002)), staining against BMP4 in a wild type mouse, and in an shh-/- mouse. The expression of BMP4 is localized in the axillary region, which is likely to undergo a higher stress. Bottom, one example of genetic description of the limb bud organization, with the regulatory pathways in which SHH is involved (from Tickle, Nature reviews, Molecular Cell Biology 7, 45-53 (2006)). The limb bud is described as having a Zone of polarizing activity (controlled by a spatial map of SHH, located in the axillary area, here in red), from which SHH diffuses, influencing the expression of Gli, and other genes. Sonic Hedgehog, has been shown to be correlated with the Antero-posterior differences in 3 fingers only. Current views do not clearly explain how individual fingers are patterned.

problem, and one has to treat the displacement vector at each point, without a priori on the possible outcome, and including physical forces. Indeed, the collinearity concept that prevails in developmental biology already assumes an oriented 1D growth pattern over which stripes of genetic expressions form (see for example Fig. 6 left). The description of growth by zebra-stripes of genetic expressions already supposes that these stripes decorate an existing "ribbon", hence they make the implicit assumption that growth is $1 \mathrm{D}$, as does Darwin with bat limb formation. This is explicitly stated, although en passant, in reputed work, such as Duboule's [73], for this author, both the succession of Hox genes along the trunk, and the genetic pattern of hands are "unidirectional". Such an oriented $1 \mathrm{D}$ growth has to be explained by the physics of the process $^{23}$. In addition, when growth occurs sideways, almost perpendicularly, an anisotropic feature, or an instability explaining lateral outgrowth has to be invoked, in order to explain physically the change in growth direction. For example: when the segments of the spine form, cells present inside the segmentation furrow tend to escape the line by

\footnotetext{
${ }^{23}$ In the same spirit, the differences in appendages forms of the fly is finely studied by marking gene expressions on the rings of the imaginal discs. However, by assuming an already existing imaginal disc, these studies only address minor differences inside a predetermined pattern of appendage whose basic plan is a set of telescopic tubes.
}

migrating away exactly perpendicularly to the segment. Therefore, the formation of the segmentation line perpendicular to one axis, induces a migration perpendicular to the axis, without a specific molecular instruction to do so, this might possibly be an explanation of the tendency of appendages to grow outwards at "boundaries" between domains of genetic expressions.

Moreover, purely geometrical effects play an important role: the shape of the SHH and of the BMP gradient around the limb bud, and its temporal action, is apparently linked to the antero-posterior pattern of the fingers [62]. However the spatial organization of these gene products is associated to a strong bending of the boundary condition in the axillary region, which is naturally a more stressed area (Fig. 11). Therefore it seems not sufficient to describe chemical actions in terms of orthogonal gradients. In the case of $s h h$, not all fingers have a shape related to shh, therefore, although shh may modulate finger shape ${ }^{24}$, it is not responsible for the existence of fingers per se (mouse without shh at all still form one finger, almost normal [71]). This shows that, in general terms, a conceptual framework allowing one to relate genetic experiments to physics of pattern formation is still lacking.

Understanding limb growth requires to couple mathematically the chemical inductions with the spatio-temporal

\footnotetext{
24 The spatial gradients are important but also the total time
} of exposure to the molecule. 


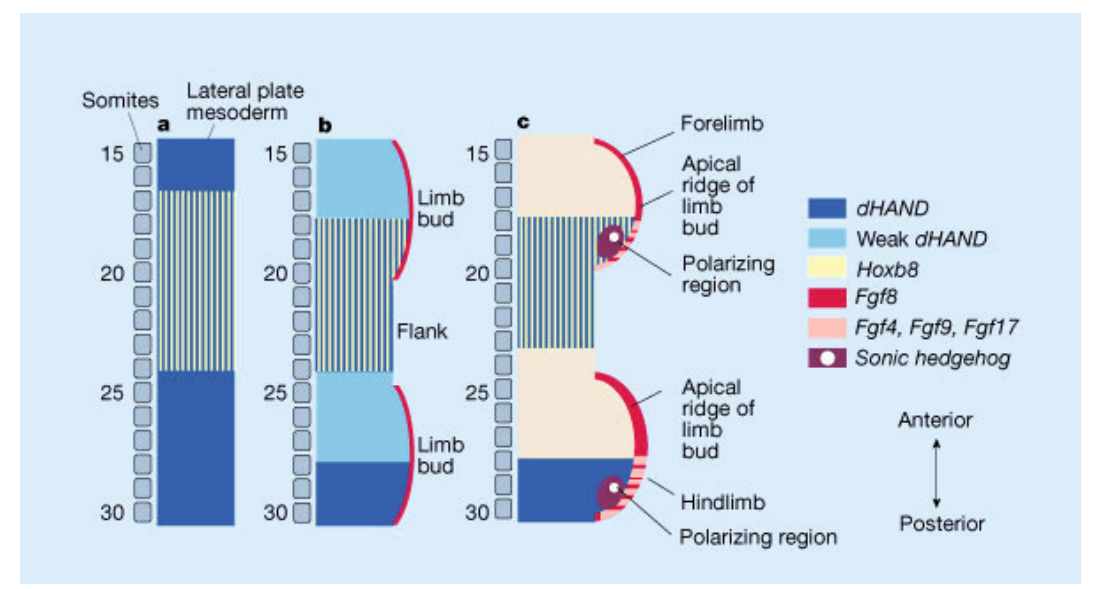

Fig. 12. (Color online) A typical description of limb bud formation from lateral plate mesoderm. From Martin Cohn [75]. The figure represents 3 stages of limb bud outgrowth along the flanks. Only the right half of the body is schematized, and only the part of the body located schematically between pelvis and shoulders. The little squares numbered from 15 to 30 represent the somites, which are precursors of vertebrate (actually there is a shift of $1 / 2$ of a wavelength between vertebrae and somites). The protruding features with a purple edge are the nascent limb buds. Such descriptions always assume an existing lateral plate (here represented by the blue areas of the body at the stage represented to the left). Ab initio (left), zones precursor of limbs exist in the form of "lateral plate mesoderm" already present. Interestingly for physicists, such an image shows a forward displacement of the limb bud boundary (purple), as a free boundary growth, but it is not said what determines the displacement field. Much like fly appendages, the description already assumes the existence of an area committed to become a limb.

field of growth, including such mechanical features as tension along curved surfaces and internal stresses, since completely different gradient maps can be obtained, in different boundary and force conditions, especially if mechanical feedback regulates tissue growth [74].

Another problem lies in experimental skill. It seems from oral interview of scientists in limb developmental biology, that, in order to produce extra limbs or extra digits, beads soaked in chemicals have to be put at specific times, and specific places, in an area called "limb field". Especially, aberrant digit distributions can be obtained by grafting pieces of limb buds onto other limb buds, but at very early stages where digits are supposed not to be present at all, therefore even the timing of digit appearance is not truly understood.

In the same spirit, there is no way an extra limb can be generated on the head or the chest, by the same soaked beads which so easily give an extra limb along the limb field. Therefore, the interpretation of the inductive events is incomplete, and requires a more detailed (spatiotemporal) understanding of what the limb field actually is.

\subsection{The limb field}

True as well as extra limbs actually originate in the lateral mesoderm ${ }^{25}$ of the flanks, in an area called "limb field" or "lateral plate" or "limb plate" [75]. While preparing this review, I found it impossible to find a description of where the lateral plates come from. All existing work assumes an

25 The early embryo is composed of 3 cell layers, the upper cell layer called ectoderm, the inner cell layer called mesoderm, and the lower cell layer called endoderm. During morphogenesis, complex motions tend to reshuffle and fold the layers. already formed lateral plate for the extension of the limb (Fig. 12), or for the early expression of limb markers (such as Tbx5). Therefore, all existing work in limb formation does not actually address the problem of limb origin, but rather, fine adjustments inside an area already committed to give limbs.

In a 2000 article [75] we can read: "However, control of limb development is apparently situated in the lateral plate mesoderm, and has been experimentally shown to be independent of an axial Hox code" but nothing is said about the origin of the lateral plate itself.

In a 2002 review, the origin of the lateral plate was presented in the following way [76]: "Moving backwards in developmental time raises the questions of how budding of the limb is first initiated and what normally serves to restrict the positions of the limb buds along the rostral-caudal axis of the body. Molecular experiments in the chick suggest that an intricate dance between FGF and WNT signaling is involved in limb bud initiation (Kawakami et al. [77]). A series of sequential signals are passed between WNT and FGF in a wave across the medial to lateral aspect of the body (somite, intermediate mesoderm, lateral plate mesoderm, ectoderm). In this dance the partners are exchanged while the overall melody remains the same. In the presumptive forelimb region, Wnt2b becomes restricted along the rostral-caudal region to the intermediate and lateral plate mesoderm. It is not yet known what genes are involved in defining the rostral-caudal domain of Wnt expression. Presumably axial patterning determinants are important, and these could include the Hox genes as mutation of Hoxb5 leads to a rostral shift of the forelimb field (Rancourt et al., 1995 [78])." 
However, Kawakami's article deals with Fgf-Wnt signaling in already existing lateral plates, or in regenerative tissue. Rancourt's article deals also with an already existing lateral plate. In a 2003 article entitled " $T b x 5$ is essential for forelimb bud initiation following patterning of the limb field in the mouse embryo" [79] one finds an important triggering effect of $T b x 5$ upon limb outgrowth, but again inside an existing lateral plate. In this article, it is stated that: "Limb formation occurs as a result of interplay between fibroblast growth factor (FGF) and Wnt signaling. What initiates these signaling cascades and thus limb bud outgrowth at defined locations along the antero-posterior axis of the embryo is not known." And also: "The definition of the fields of Lateral Plate Mesoderm that will give rise to limb buds occur possibly as a result of patterning by Hox gene activity along the AP axis of the vertebrate embryo (Cohn et al., 1997; Cohn and Tickle, 1999 [51]; Popperl et al., 2000 [80]; Rancourt et al., 1995 [78])" However, Popperl et al.'s article deals mostly with the role of lazarus in the cranial (hindbrain and anterior trunk) compartment, (especially it shows how $p b x$ genes participate in the regulatory loop of hox genes needed to specify rhombomere boundaries in the hindbrain) and when coming to limbs gives the same evidence that Tbx5 triggers forelimb outgrowth, since $l z r-/-$ zebrafish do not show fin rudiments at all, and no Tbx5 staining. There is no rationale about limb positioning, apart from a correlation between absence of lazarus, and absence of fins. Especially, it is quite clear that the fin rudiments appear as round balls on the side of the flanks (Fig. 6. I in Ref. [80]). No mechanistic explanation is given of how a collinear series of genes along the neural crest might induce lateral formation of roundish masses of cells (the fin precursor). Popperl's et al. article deals with deletion of hoxb-5 and hoxb- 6 genes, and shows only a very modest shift of the shoulder girdle of about one or at most two vertebrae, when hoxb-5 genes are deleted and no mechanistic explanation. It is difficult to understand from this work in what respect hoxb-5 might be related to limb "positioning", since when totally absent, there is a normal limb, which is "positioned" by something, and the position is almost normal, except that it is slightly shifted from the wild type. Cohn's et al. 1997 shows the opposite of what is claimed (if hox genes play a regulatory role in the lateral plate, it is independent of the AP polarity), and Cohn and Tickle's 1999 article deals with limblessness in snakes. While this last article may give a clue of why snakes do not have limbs, it does not give a hint of why, in tetrapods, limbs would appear where they appear (stated otherwise, they correlate limb position and domains of hox genes, but what serves to position the domain of expression, in the first place?).

An other article shows a correlation between Hox9 genes and limb positioning, but there is no mechanistic relationship between limb positions and Hox9 genes expression [81]. To make a long story short, in a 2006 review [82] we still read: "The first problem in human limb development is not outgrowth but locating the point of outgrowth along the body wall. Little is known about the

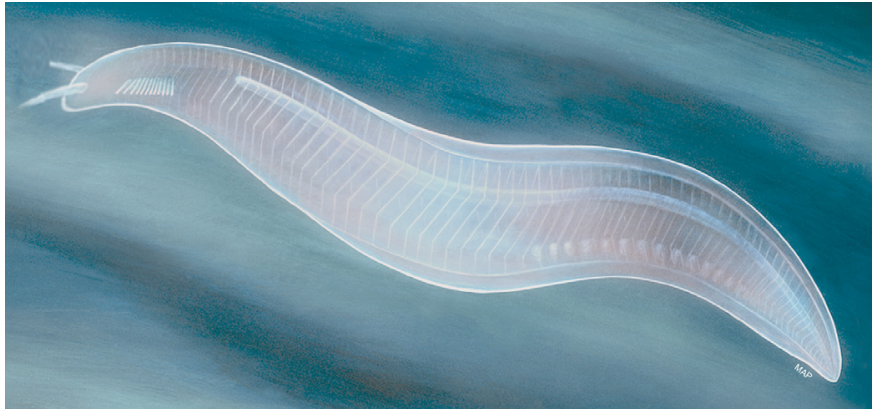

Fig. 13. (Color online) The earliest known ancestor of the vertebrates, the bilateran, segmented Pikaia 550 My (C)Mary Parrish/The Smithsonian Institution).

exact mechanism, but globally acting genes such as Hox 9 and Tbx4 seem to be crucial for hind limb placement. They are expressed in the lateral plate mesoderm before outgrowth." A sentence which again already supposes the existence of lateral mesoderm.

The review presented here highlights that inductive cascades of unidirectional gradients of scalar quantities cannot explain morphogenesis. In effect, if morphogenesis were organized by successive discrete inductions by feedback loops along the slopes of scalar gradients of morphogenes, the animal shapes would be possibly arbitrary, without geometrical constraints, and animal forms would truly be random. But, as has been recognized for long, and quantified more accurately lately, the body axis and limb fields are actually organized by a hydrodynamic-like flow, in which a segmentation wave propagates. Such that being a forelimb or being a hindlimb, is actually a matter of geometry of a deformation field advected by a flow (See Sect. 7), which serves as boundary condition for genetic expressions.

\section{The paleontological point of view}

\subsection{Tetrapod origin}

If we turn to paleontology, we find a description of tetrapods appearance into three main steps. Appearance of chordates, segmentation of lateral fins, appearance of tetrapods. The chordates are the class of animals comprising vertebrates and a small number of jaw-less, vertebrateless fish like the amphioxus (lancelet) ${ }^{26}$ and the lamprey $^{27}$ [83]. The first fish-looking, but jaw-less chordates, date back to the Cambrian era, about 540 millions years ago. At the Cambrian explosion a number of complex bauplan of multicellular organisms appeared very rapidly [84], among which the first chordates, presumably Cathaymirus diadexus [85] or Pikaia gracilens [86] are our ancestor (Fig. 13).

\footnotetext{
${ }^{26}$ A cephalochordate with a notochord but no clear vertebrae segments.

27 A fish with a circular plunger-mouth, and vertebrate-like segments.
} 


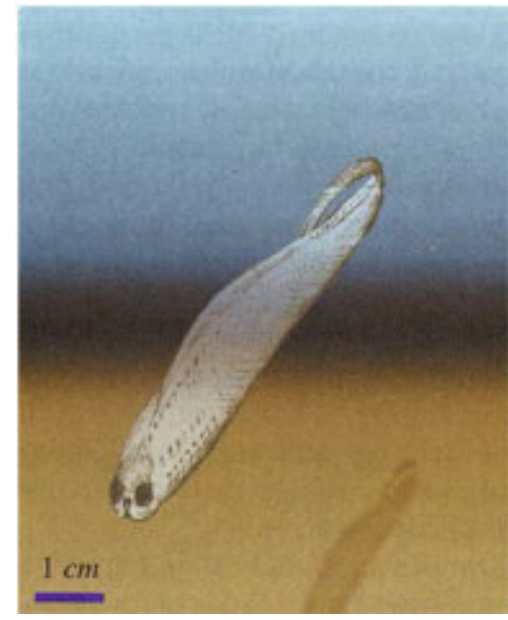

Fig. 14. (Color online) The first true chordate, Haikuichtys (470 My).

As a transition towards true vertebrates in the fossil record, appears haikuichtys [87] (or Myllokunmigia Fengjiaoa) (470 My), of the lamprey family (Fig. 14).

From then on true fishes are present in the fossil record. Early fishes seemed to have fin folds running along the body like curtains. Although there also exist specimens of fishes with rudimentary paddles below the jaw, as early as approx. $400 \mathrm{My}$, Ateleaspis tesselatta [88].

Somehow, the elongated fins present along the body "became segmented", restricted to some areas, and the lateral fins became recognizable as pectoral and pelvic fins. These fins were essentially paddles with rays fanning away from the body, as observed on so many common fish. One finds then along the fish body: the caudal fin, the anal fin, and the dorsal fin. Simple inspection of these appendages show that they seem to be continuations of existing folds, which may be more or less elongated. The dorsal fins seem to drift caudally during development, as the tail extends.

Progressively during evolution, some families of fish disappeared, other appeared. Fish body became separated into several forms, bony fishes and non-bony fishes, agnaths (fish without jaws) and gnathostome (with jaws). Among the non-bony fishes, one finds the class of sharklike fishes, which include flat fishes like the torpedo and ray-fish (class Chondrichthyes, $370 \mathrm{My}$ ). The usual bony fishes, said Osteichthyes appear 395 My ago. There exist three kinds of bony fishes, the ones with ray-finned paddles, known as teleosts. They form the largest and most common group of present fishes. The second group is the lungfishes. The other group, from which tetrapods descend are the Sarcopterygian fishes. Please refer to a general textbook on vertebrate evolution for more details.

The Sarcopterygian fishes had well developed lateral fins, with a paddle located away from the body at the apex of a fleshy limb ("lobbed fin"). Around 350 My from now, a group known as the Crossopterygian (among which the celebrated Latimera chalumnae, known also as coelacanth) evolved limbs, which allowed them to conquer the land, as amphibians. Actually, it is well admitted that limbs appeared before fish were able to conquer the land,

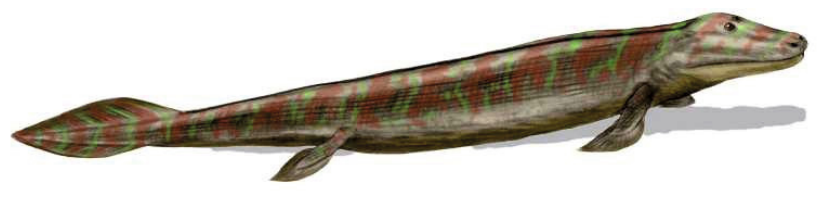

Fig. 15. (Color online) The first known tetrapod, Tiktaalik, (c)Arthur Weasley 2007.

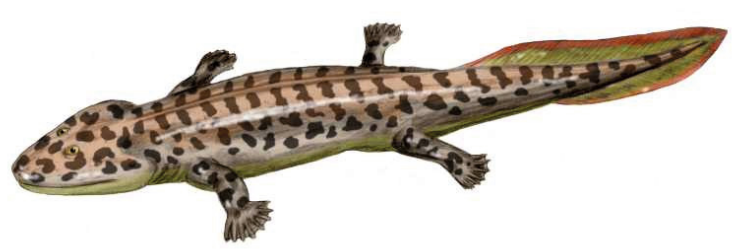

Fig. 16. (Color online) The first known tetrapod having an autopod (a "hand") instead of a lobbed fin, Acanthostega, (c)Arthur Weasley 2007.

and that early tetrapods had an amphibian life in swamps. This is a likely feature of Darwinian evolution in some instances: useful traits in a new niche, have first to appear in another niche, where they may not be as useful (see explanation by Darwin on this point in the chapter IV of his opus). There still exists a fish with well enough developed "limbs" to be able to run, quite rapidly, on the land (goby fish (Proterorhinus) [89]), and there also exist fishes with lobbed fins that fortuitously "walk" the bottom of the sea. However, these fishes are known not to be related to the ancestors of tetrapods in any straightforward manner [90]. Whatsoever, the first known tetrapod is an elongated animal somewhat crocodile-looking, Tiktaalik roseae [91] (Fig. 15), dating back 370 My. These early tetrapods have well formed complex limbs apparently almost "right away". The number of "iterations", i.e., the number of significant mutations altering the bauplan between a fish and a tetrapod may as well be very small, a fact confirmed by recent genetic analysis (it has been shown quite recently, conforming common beliefs up to this date, that the distal rays of the fins - the fin blade - are genetically akin to fingers [92]).

From there on, progressive evolution has slightly modified the tetrapods, of course, but over a fixed plan (tail, limbs, head, backbone). The first known tetrapod to have fingers is Acanthosthega [93] (Fig. 16), which had eight fingers.

\subsection{About pentadactily}

The early tetrapods had a count of digital rays or of true fingers much higher than later tetrapods [68], and a progressive reduction in finger count was observed, until the pattern was stabilized to the well known pentadactylous pattern, composed of five fingers, which is almost universal in present tetrapods. The origin of pentadactily is not 

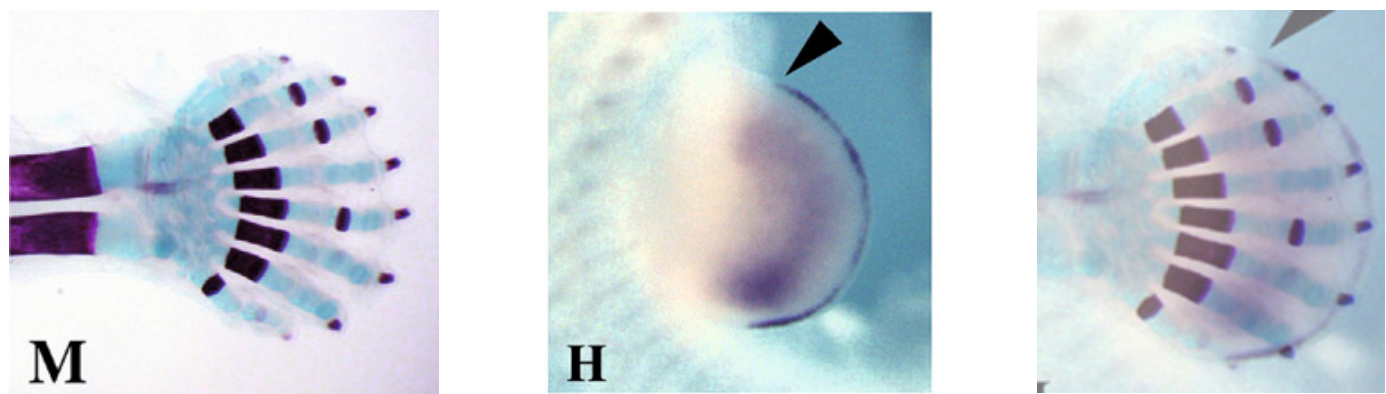

Fig. 17. (Color online) Reprinted from reference [69]. A mutant mouse (gli3-/-) having 9 digits in its digital arch ("M" in the original caption). The hand shape reflects the shape of the early limb bud ("H"), and the number of digital rays equates (in the background) the number of somites at the connection between flanks and bud. The figure to the right shows an overlap of the hand, at the stage "M" of the growth, over the initial limb bud along the flank, showing that the digital arch spans exactly the early form of the limb bud (image obtained by centering, scaling and averaging $\mathrm{M}$ and $\mathrm{H}$ ), with the distal-most phalanges following exactly the apical ectodermal ridge, where nails form. The convergent nature of the rays in the wrist area creates a complex morphogenetic pattern for the wrist. In addition, the set of carpals on which the fingers form seems to be bent by previous morphogenetic movements [73].

known, unless related to complex genetic inductions in nested form; especially, pentadactily has been related to the expression of Hoxd genes [91]. These are expressed in the caudal part of the embryo, including hindlimb territory and "surprisingly" also in the forelimb. As stated by Tabin, there may be 5 "addresses" for finger type, in the form of available Hoxd genes. However, these genes may specify fingers identity, but not finger count. From a point of view of evolution, it is assumed that a limb with five digits is easier to flex [94]. It is clear that finger count is not a matter of each finger being individually fixed, since deletion of a single gene is able to jump the finger count from 5 to approximately 10 in a mutant mouse [69]. It is more likely that the finger count is a matter of an instability wavelength, as first suggested by Shubin et al. [68], and that modifications of physical parameters in the process of cartilage condensation [67] will generate more or less fingers, with possible period doublings as for the Gli3-/mouse $^{28}$. This kind of period doubling is also present in fish fins, for example Figure 20 shows a fish stained against hox genes present in limbs of tetrapods. These genes are present in the fins. However, a careful count of rays in the fins, shows that they correspond to a period doubling with respect to the vertebrae (seen also in the most common fish).

Direct inspection of the limb paddle (Fig. 8) shows that it finds itself in front of five vertebrae precursors (somites), at the moment of outgrowth, therefore, the digit count may be just a matter of mode locking of finger count onto vertebrae wavelength, in the early limb field, which extends in the form of the well known "digital arch". The digital arch is the arch-like structure formed by the fingers, located distal to the carpal bones (to which fingers articulate), this "arch" spans the fingers and the tissue in between (the tissue that exists prior to cellular death

\footnotetext{
28 This is also apparent in the fact that massive elongation of the limbs is associated to reduction of finger numbers, often by loss of vestigial fingers on the sides of a central unique finger (unguligrades, see Fig. 1).
}

that frees the individual fingers in humans). If one looks carefully at the phalanx segmentation, and at the troughs between fingers, one recognizes a striking regular pattern, which has an arch-like shape (Fig. 17). For example the forelimb pattern of the gli3-/- mouse which has 9 fingers is that of a regular fan of digital rays forming an arch. This arch overlaps exactly the shape of the early limb bud, at the moment when it outgrows from the flanks, and this number corresponds to the number of vertebrae precursors located parallel to it.

This is also apparent in the fact that massive elongation of the limbs is associated to reduction of finger numbers, often by loss of vestigial fingers on the sides of a central unique finger (unguligrades, see Fig. 1).

Therefore, understanding the distribution of bones in the body, especially in the limbs, is a matter of coupling the formation of the body, with its limb fields, to the segmentation wave which propagates the formation of cartilage condensations. As this wave descends along the body to form the successive vertebrae, it interacts sideways with the limb field, at a very early stage, to induce the pattern of bones of the limbs, anal fin, dorsal fin, etc., in relation to collinear expressions of Hox genes which gently modify the shape of each ray, which are otherwise all identical at start. This is probably the meaning of the sentence above "In this dance the partners are exchanged while the overall melody remains the same".

\subsection{Fold extension}

All fin-like appendages, be it anal, pelvic, pectoral or dorsal seem to be progressive extensions of superficial folds. Therefore, it seems that folds have a natural tendency to grow away as if being "a fold" would suffice to induce easily more development. The fold geometry implies great shear on cells, therefore stimulating growth factors. This is because along a fold, the deformation field is in traction in the convexity and in compression in the concavity. Cells located right at the fold edge are quite sheared. 


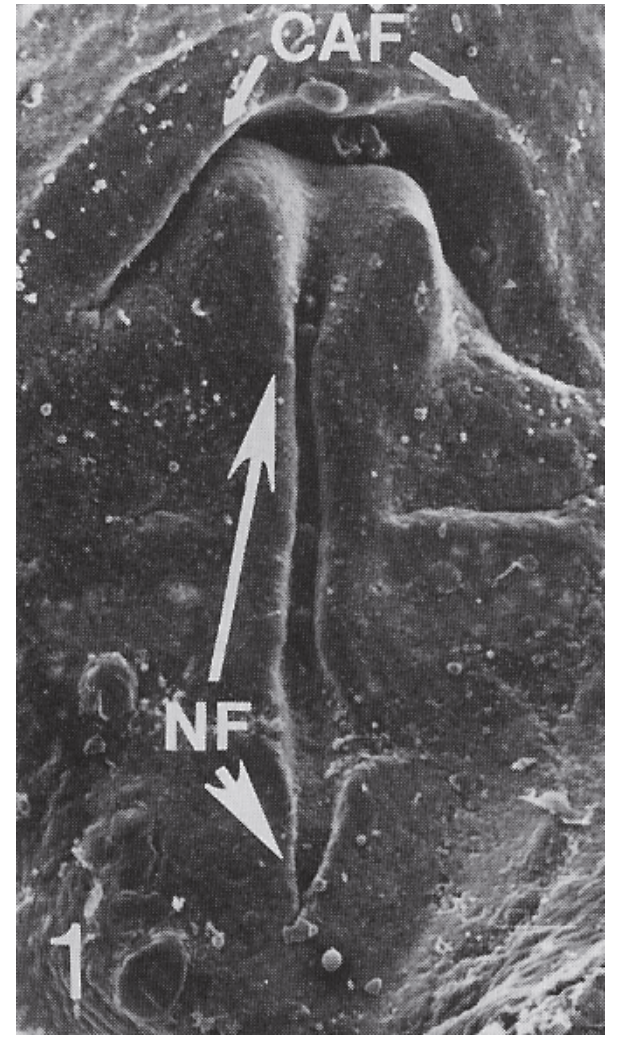

Fig. 18. SEM photography showing the "pocket" like fold of the chorion which is bound to shroud the entire embryo body $(\mathrm{CAF}=$ chorio amniotic fold; $\mathrm{NF}=$ neural fold $)$. This pocket forms by a forward dive of the embryo brain folds From reference [96]. See also database GEISHA [97] neuropillin2, fgfr2 stainings and others.

The Apical ectodermal ridge is, in this respect, a fold. It is well known that compression and stretch forces tend to stimulate growth factors and cell migration (see Sect. 6), and have a considerable structural and dynamical impact on fibroblasts ([95], and references therein). This might be the natural explanation of the tendency of fold edges to grow out, in the shape of fins, ear lobes, trunks, tails, etc.

The first example of large fold which has an accelerated growth is actually the chorio-amniotic fold of the amniotes $^{29}$. The amniotic sac is a pouch which surrounds certain vertebrates during development (the amniotes). This pouch forms by rapid growth of an edge fold located just ahead of the head, and caudal of the tail [96]. This pouch starts as a pocket-like fold, which eventually shrouds the entire animal body (Fig. 18).

Physically, it seems to me that as the extremities of the embryo move more asunder, they tend to fold the surface on which they grow, as the embryo tip so to speak "dives" and curls downwards. As soon as it is formed, the lip of the fold undergoes a tremendously rapid growth (a few hours),

\footnotetext{
29 Amniotes are vertebrates which developed an amniotic sac around the embryo, thus providing a protected liquid environment for the development of the embryo (reptiles, birds, mammals are amniotes), the amphibians are anamniotes.
}

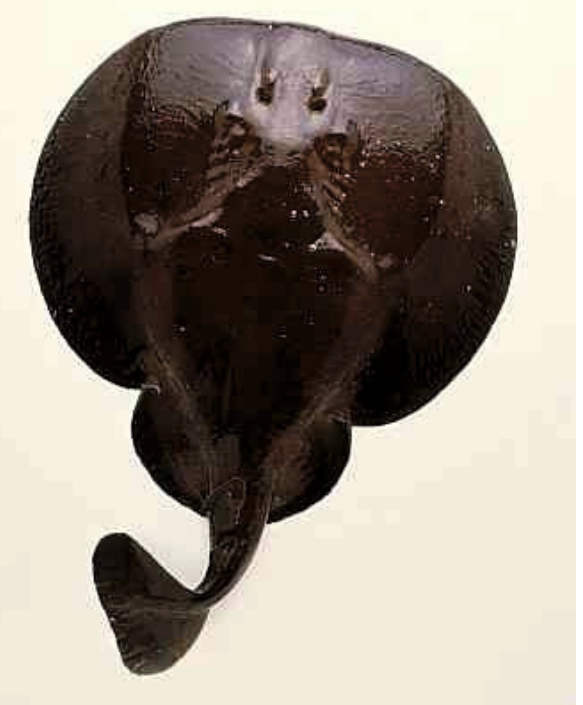

Fig. 19. (Color online) Torpedo fish (c) DK Images.

until it surrounds the entire embryo. The final point of closure is located above the navel area.

As a consequence, the transition from anamniotes to amniotes may be a matter of a one-or-nothing physical event: either the fold forms, and it grows quickly until completion of the amniotic sac, or it does not. In this spirit, evolutionary "bifurcations" correspond actually to bifurcations in the usual physical sense.

The origin of the amniotic sac may be tracked back to the fact that the embryo has a "downwards", ventral, curl $^{30}$ so that it tends to fold its substrate. The fact that amniotes are characterize by having an amniotic sac, and developmental differences in the head (more pronounced skull and orbits) makes sense: a more forward dive of the head rudiments give more likely an amniotic sac, and larger head features. The chorio-amniotic fold is analogous to a fin fold, except that the topology of the edge is a circle which closes up progressively like an iris, to form a hollow bag, and not a flat veil. I believe that a genetic analysis of the amniotic edge would give similar genetic inductions as a tail (see data base GEISHA [97]).

Nevertheless, when folds growth is prolonged by evolution, it is so in the direction of the existing fold. Stated otherwise, if the appearance of a fold corresponds to crossing of a generic biomechanical threshold for folding, one may expect that the fold is itself stable in an open set of parameters, such that, "conserving the scaffold", as stated by Darwin, a fold may elongate or shorten during evolution. This is to say that the more or less elongated aspect of rays in fins is somewhat predictable, it suffices to push forward the pattern of fish bones in the rays. This is also true of other edge-like organs, such as ears, nostrils or lips, which are well known to be observed in nature with almost "arbitrary" lengths, a word often used by Darwin.

\footnotetext{
${ }^{30}$ We shall see below that the curl (Sects. 6 to 9 ) is a physical consequence of vorticity.
} 


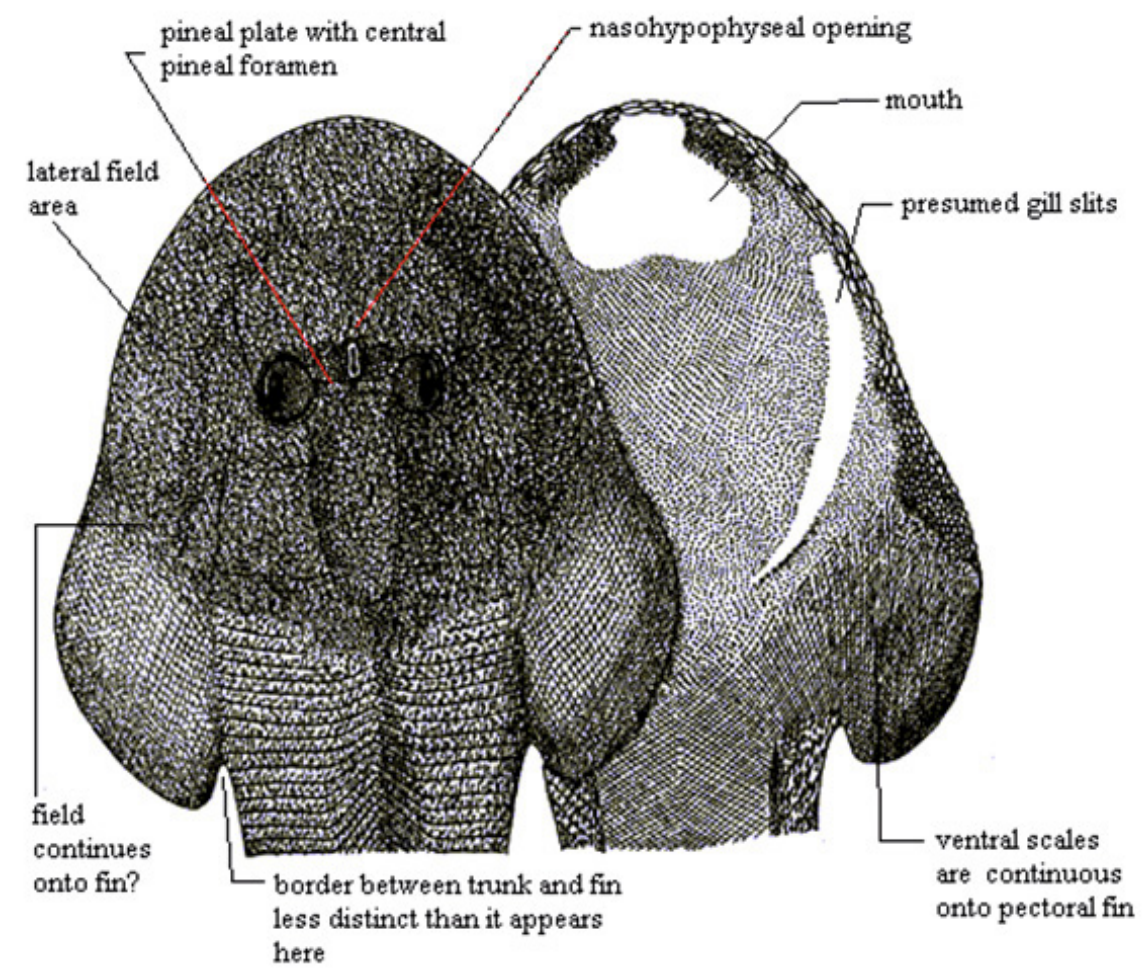

Fig. 20. Ateleaspis tesselatta headshield and pectoral fins in dorsal and ventral views (from Ritchie 1967). Early fish, like Ateleaspis tessellata have limb paddles below a head which has a typical inverted horseshoe shape. The limb paddles emanate from a bent area forming axils. As explained below, this body organization is typical of a trail, as formed by the visco-elastic deformation of the early blastula.

In the case of the pectoral and pelvic fins, they are believed to originate from a uniform fin present along the body; in the centre of this fin, an area was interrupted or somehow inhibited. As a central portion of the lateral fin disappeared, the limbs appeared as fin folds restricted to a more anterior and a more posterior part, but initially connected. This interruption is visible in torpenid fish (Fig. 19) which, although they do not have actual limbs, have lateral fins which are interrupted, in the shape of an "8-shape". This is also true of the dorsal fin.

One may imagine that a similar interruption occurred in early fish, that led to more restricted fin-folds, giving rise to actual limbs, instead of the large veil of torpenids. How this happened is not known, but it may imply a deformation field leading to a constriction in the centre of the animal, and an expansion in the anterior and posterior parts. As stated by Tabin [93] "As fins evolved, the fin field contracted so that the posterior Hox genes are no longer expressed in a strictly contiguous pattern in the hind limb and the flank." In the same spirit, extra limb experiments lead Mikiko et al. [65] to write "the potential to form limbs is much more widespread throughout the body. This may be a legacy from ancestral vertebrates from which present day vertebrates evolved." This is to say that it is not the regulatory loops of the genetic expressions which actually induced the splitting of the fin into limbs, but some "contraction", which remains to be understood. With the same genes, a contracted veil gives two limb areas, forelimb, and hindlimb, but the rest of the flank could as well have produced a limb.

One also finds in the fossil record fish with reasonably well formed limb buds, which hardly evoke an interrupted lateral fin [88]. It seems as though some twist or engulfment occurred in the area of the axils (Fig. 20), the bud itself rotating towards the median line, while the headshield rotates away from the median axis in the form of a trail.

\subsection{Limb extension}

Genetic analysis, in relation to evolutionary issues, shows that actually, genes for limbs and for tails are similar, and many are identical ([98] and references above ${ }^{31}$, Ref. [60]). Also, genes which serve to form the true limb skeleton, are actually present in fish fins, in which such skeletal elements are absent [98] (Fig. 21).

Therefore, the situation in this field is not quite clear. It seems again that the same genes, already present prior to limb appearance, may serve to make a tail, a fin, or a limb, depending on where they are expressed, i.e., depending on the local geometrical context, and physical twists which occurred before genetic expression, in addition to

\footnotetext{
31 "Not only are the same Hox genes expressed in both developing appendages but they are expressed in identical spatial and temporal patterns" in reference [85].
} 

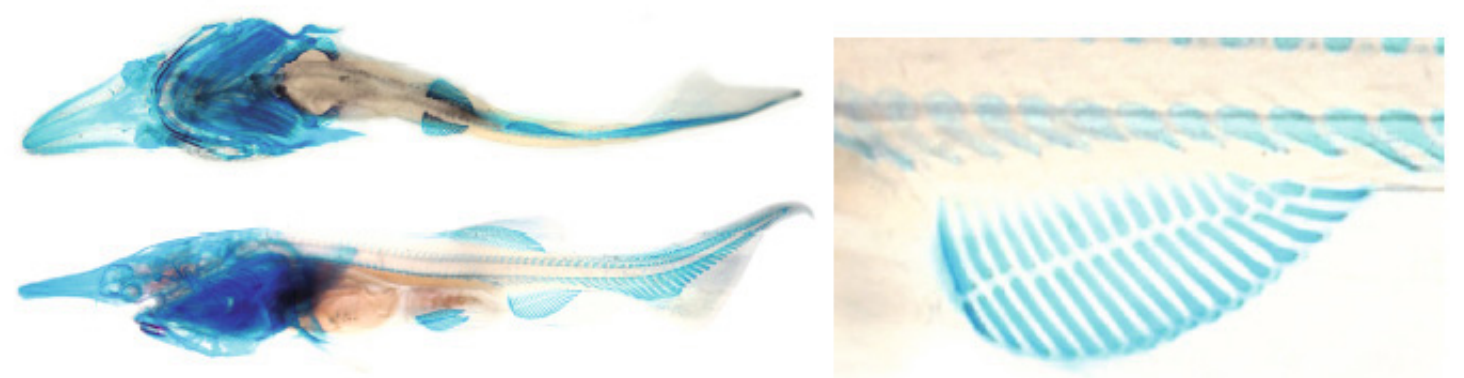

Fig. 21. (Color online) Left. Fish stained against Hox genes [98], present during limb development. Such genes are found in fins. Count of wavelength in the area of the fin rays (right) shows a period doubling between rays in fin folds and vertebrate count along the dorsal axis. (original legend: Underside and side views of a juvenile specimen of the North American paddlefish, Polyodon spathula, stained for developing cartilage. Photo by Davis).

chemical differences. This reminds of the fact that cnidarians and vertebrates have similar gene regulatory networks. The inductive cascade is not just a mere nuclear induction following strictly the blueprint of some Hox genes. The dynamic, spatio-temporal features are crucial. It is easy to imagine that the same genes may grow a tail, along the median axis, because of absolute symmetry, while they will grow a limb sideways, because of the broken symmetry. There is no doubt that exactly the same genes may give totally different physical realizations, depending on boundary conditions, since a left and a right limb are totally different, but made with the same genes. In this view, the left and right limb are the chiral outcome of the same process which, when non-chiral, gives a different "limb", which is called "a tail".

Later during evolution limbs disappeared from some tetrapod lineages which became aped, such as snakes. Snakes follow lizards, in evolution $[48,99]$. However, many snakes still have vestigial limbs (boa, python [49]). A few years ago a fossil was found of a snake Eupodophis descouensi with extremely small vestigial hindlimbs [100]. Therefore it seems that the natural pattern of "tetrapod" limbs is 0,2 or 4 limbs. When there are 4 limbs, 2 of them are found above the navel, two below. Hindlimbs are generally larger, suggesting a natural tendency of body morphogenesis towards bipedalism, which is actually quite frequent, and always on hindlimbs. Hindlimbs form first during development of most tetrapods, and they are likely to be last to disappear, as known in snakes. The fact that hindlimbs form first and are thus often larger, may be ascribed to the narrow spacing in the caudal part of the early embryo (more below).

Many animals without limbs have limbs at an embryonic stage, just as we humans have a tail at embryonic stage. For example, hindlimbs seem absent of cetaceans, but the disappearance of the hindlimbs in these genera is in fact a progressive absorption of the hindlimbs by the growing body at late stages of gestation; cetaceans have hindlimbs at an early embryonic stage. It is even a classical observation that dolphins and whales, which do not normally have pelvic fins, will be spuriously found with perfectly well developed pelvic fins [101]. In humans, having a well formed tail, sometimes as long as thirty centimetres, and mobile, is a very rare, but well documented condition [102]. These tails may be formed of soft tissue only, or may contain vertebrae. Needless to say such an appendage is generally severed by medical doctors at birth, although there is no obvious reason to do so, except human prejudice.

Therefore, in tetrapods, limbs are "always there", topologically present, and so is the tail, and they may or may not grow out, depending on the correct supply of many candidate triggering molecules, they are "latent". However, if limbs do grow, they only grow in the area of the normal limbs of tetrapods, and nowhere else, with possible modest shifts along the body, just like a tail grows or not, but always in the area of the tail, and nowhere else. We shall see in Setion 7 why this is topologically fixed. (NB: I do not discuss in this review all the unfortunate teratologies seen in pediatrics which would deserve a review in themselves. Especially, the Siamese twins are generally a consequence of two embryos progressively fusing. Several famous cases of newborns with, for example up to six hindlimbs, are actually cases of co-joined twins in which the entire body of one of the twins was absorbed by the other - a situation known as foetus in foetu, (see the recent famous case of Lakshmi, the Indian baby girl born with four legs and four arms [103]).

\subsection{Limb flexion}

An additional fact concerns the flexion of the limbs. In all vertebrates, hindlimb and forelimb flex in the opposite way (legs flex downwards after the knee, arms flex upwards after the elbow) $[95,104]$. It is assumed that genetic differences between the set of genes at the hindlimb, and at the forelimb must have evolved from an initial identical set which allowed this specific difference to be obtained by a progressive drift after duplication [105]. Some attempts 


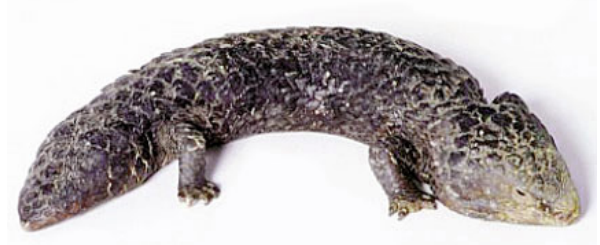

Fig. 22. (Color online) Lizards, and especially some shingelbacks, have almost perfectly symmetrical limbs. In tetrapods, if a left arm is considered as "left handed", then the left leg handedness, is actually a right handedness. It may be possible that in addition to the exact left-right symmetry, there exists an antero-posterior partial mirror symmetry, around the navel. Limb position would be decided by a mirror symmetry round the navel instead of an antero-posterior gradient of chemicals.

have been made to modify limb flexion by genetic means, with only one case of significant success.

The wrist flexion may be very modestly modified [105] by genetic transfections by Pitx 1 (which is normally present only in hindlimbs), but the origin of limb flexion at the knee or elbow joint remains somewhat mysterious. The concept that gene duplication was followed by a slow drift of genes allowing the direction of flexion to radically change may as well be erroneous. Although it is believed that genes must have evolved away to reach the opposite flexion, it must be observed that ancient animals, like lizards or crocodiles, have much more symmetrical limbs (with complete opposite flexions) than say, humans, while the opposite would be expected (Fig. 22).

As stated in introduction, left arm, and right arm have opposite flexion, in terms of chirality, although they are made with identical genes. The difference between the flexions of left arm and right arm are not ascribed to slow genetic drifts, but to a symmetry of the boundary conditions. Therefore, it may be possible that the difference in flexion of legs and arms may be a consequence of boundary conditions, instead of a specific set of genes. The same genes, when "duplicated" from the tail, towards the hindlimb field, and forelimb field would "automatically" give limbs with opposite flexions in these areas [16].

It should be insisted, here, on an ambiguity about "limb identity". Being a forelimb or a hindlimb defines the "limb identity". However, some animals have very different limbs, in addition to a modified flexure, between hindlimbs and forelimbs. Especially birds have wings instead of legs as forelimbs. It has been proposed that some genes esp. Pitx1 was a "determinant" of hindlimb identity [106] since that gene is not expressed in forelimbs. Strong statements are found in the literature such as "Pitx1 determines the morphology of muscle, tendon, and bones of the hindlimb". However, when inspected in detail, this sort of work shows generally that the limb flexion, this is to say, the over all chirality of the limb, is independent on such genes. These genes seem to determine more the "type", in the sense of legs vs. wings (although expression of such genes in the "wrong limb" may modify the shape of bones). Indeed, DeLaurier et al. [106] were able to generate forelimb bones resembling hindlimb bones (e.g. elbow joint looking like knee joint), but without affecting the direction of flexion of the elbow. In reference [105] the authors have shown that it was possible to somewhat alter the development of the avian wing (forelimb) to make it ressemblant to a hindlimb, but again, when looked in detail, this sort of work shows that the wing may be reverted to "a leg", but not a hindleg, a foreleg. In two articles which appeared back to back $[59,107]$, experiments of transfection of forelimb buds and hindlimb buds with Pitx1, Tbx5 and Tbx4 show generally alteration of legs towards a wing type, and vice-versa, but the limb flexion is not altered in any of the data presented in reference [106], and possibly only in a few percent of the experiments shown in reference [59] (see Fig. 1b). Moreover, in reference [107], when a wing is induced in the leg area, it is not a normal limb, but a wing with complete mirror symmetry. This is to say that a wing can be indeed obtained, which has all the flexions of a leg, but it is not that the left wing is flexed in the other direction. The significance of the reversed flexion of the hindlimb in $10 \%$ of the experiments reported in reference [59] is unclear since the electroporation experiment used to insert Tbx5 in the hindplate prior to hindlimb growth has a polarity in itself. If this experiment would be confirmed, it would be an uncommon case of a chirality, directly induced by a scalar non-chiral field. This would suggest that Tbx5 codes for a chiral molecule.

As far as the author can judge, there is no way a chicken wing may be exactly transformed into a chicken leg genetically, and vice versa. It seems that, indeed, the gross pattern of the limb is actually a matter of geometrical context, and not of genes.

The limb identity has also been correlated to collinear expression of Hox genes from proximal to distal, however, as stated by Zakany and Duboule [44]: "Only the removal of either HoxA or HoxD leads to a detectable phenotype, which is not drastic and mostly affects the digital plate. However, the combined deletion of both HoxA and HoxD leads to an early arrest of limb growth, pointing to a large functional redundancy between these two clusters". This is to say that entire clusters of Hox genes, containing up to 11 genes, which are normally present during limb development can be annihilated, with very, very, minor effects on limb shape (see Fig. 1 in Ref. [44]), corresponding to an interruption of the one-directional growth, at some point along the growth, which acts as cursor. This suggests again that limb development may just be "something else", than a genetic induction. As a concluding remark, let us note that despite a tremendous and constant attack on all nuts and bolts of the genetic construction of vertebrates, an animal having regularly well positioned ectopic limbs (say a vertebrate hexapod or octopod) was never obtained ${ }^{32}$. More specifically, the announcement of a mutation that is able to generate additional limbs shows that such limbs are actually associated to a duplication of

32 Of course, absence of evidence is not evidence of absence. 
the entire body axis, with the corresponding movements of engulfment and segmentation which create the normal limb plates [108].

\section{Developmental description of the formation of the vertebrate bauplan}

\subsection{Description of gastrulation motions}

Understanding vertebrate development requires to understand the spatio-temporal aspect of this phenomenon. This is almost absent from the genetic inductions description, and absent from the paleontological approach. Therefore one has to go into the description made by developmental biologists. A typical vertebrate development, such as development of avian or human embryos is described in embryology textbooks in the following way. The maternal ovocyte, although spherical, is not completely homogeneous, it has a bilateral symmetry breaking. When fertilized by a spermatozoid, some rapid rearrangement of the cytoplasm occurs. Specifically, the cytoplasm rotates slightly in the direction of the point of entry of the spermatozoid [109,110], ("cortical rotation, ... something purely physical and macroscopic is involved in this stage of development [109]"), such that the ovocyte acquires also an antero-posterior symmetry breaking Although important and interesting, we shall not go into the details of the fertilization process, and of the earliest events. As the first few hours pass by, the ovocyte divides, and becomes a roundish mass of cells called "morula" [111]. It is already clear that the early cleavages respect basic symmetry principles, and that there exist important mechanical aspects to cell walls equilibria. Especially, due to the arrow of time, not all cleavages are identical, thus creating a physical polarity in the early morula. More specifically, the morula inherits from the earlier cortical rotation a gradient of cell sizes, the cells being smaller towards the caudal pole. Now, the growth of the morula progressively transforms it into a larger mass of cells of a few tens of thousands of cells, which is "formless", i.e. either discoidal for animal eggs which have yolk (megalolecithal eggs), or round for the general case (amniotes or anamniotes). This formless embryo is called blastula.

At an early stage, the cells of the embryo (blastomeres) can be separated and they will each generate a complete animal $^{33}$. This was shown in historical experiments by Driesch [112] (on the urchin) and Chabry [113] on the frog. Therefore, the morphogenetic instructions seem to be positioned at later stages, in the blastula.

When the blastula reaches about 20000 cells, a massive reorganization called gastrulation [114] occurs, after which the embryo is truly called an embryo, and it has a recognizable shape (a head rudiment, a tail rudiment, a body axis, limb rudiments, eye cups, etc.). During gastrulation, and after, splitting the embryo in several parts is out of the question: a pattern is formed inside the embryo which is no longer plastic, except for some intriguing facts

\footnotetext{
${ }^{33}$ Not true in fish.
}

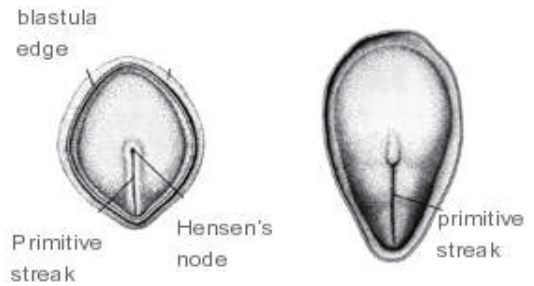

A

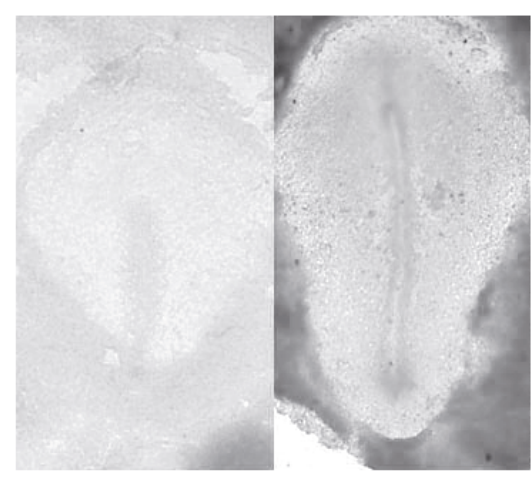

B

Fig. 23. (A) Situation in the human blastula, at the moment when the primitive streak forms (left) and then starts to recede (right). To the left the early blastula, with the primitive streak moving forward inside the blastula. Meanwhile, the blastula deforms to become elongated. Then the apex of the primitive streak recedes, while a fold appears immediately afterwards ahead of it, and the blastula elongates even more caudally. (B) actual images in the avian embryo (from database GEISHA).

(e.g.: nose bud, if transferred to the flank, will induce a limb, reviewed in $[62]^{34}$ ). The motion that establishes the body plan from a formless disc is very rapid (about $12 \mathrm{~h}$ in the chicken), it occurs when the embryo is very small (about $4 \mathrm{~mm}$ ), it encompasses the entire blastula, and after that event, a topologically complete, although not finished animal is formed. The reorganization is so complex that it is very difficult to follow where exactly each cell goes. Developmental biologists build up fate maps ${ }^{35}$ which are quite complex, and clearly non-connected. A number of wrong views have spread due to difficulty of doing such fate maps $[115,116]$. Retrospectively, as we shall see that the body organization is a matter of cross-product of a rapid flow by a buckling folding, it is natural that constructing fate maps is a difficult and awkward task. After gastrulation, the embryo is complete in that all parts exist rudimentary, although each part is far from finished yet. The process may be called top-down: the gross plan is established first, then the details in each corner.

\footnotetext{
34 When an otic vesicle or a nose bud is transplanted to the flank, it triggers growth of a limb. This is ascribed to the fact that they produce signalling molecules of the FGF family. However, neither triggers a limb in the head area, therefore, there is "something else".

35 "Fate maps are the projection of advanced developmental stages of an organism back to an earlier stage" [102].
} 

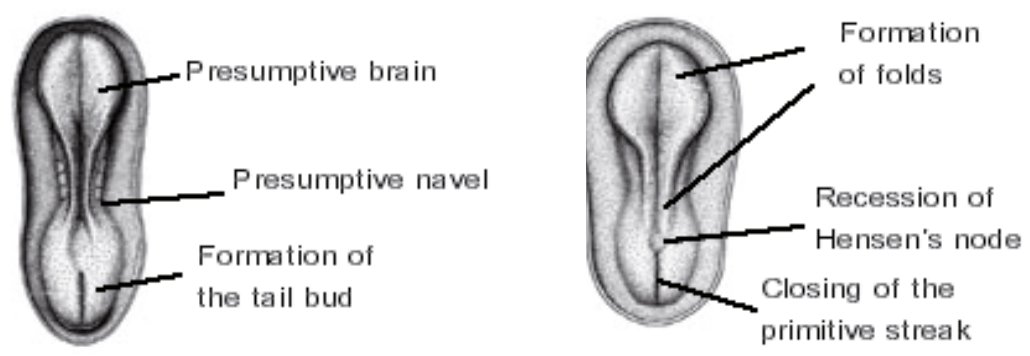

Fig. 24. Continuation of the growth from Figure 23. Hensen's node keeps on receding southwards. Folds form just above Hensen's node, as this one recedes. As the folds get more marked, the entire blastula adopts a "Figure 8" shape and the embryo starts to exhibit a mirror symmetry around the navel region. The folds in the anterior region are the neural plate, the folds in the posterior region form the tail bud.

In more detail, the gastrulation proceeds this way: the flat roughly discoidal bi-layered sheet (called blastula) undergoes a motion towards the south of the disk. As it does, it deforms to become more elongated caudally, and a furrow appears which progresses up to a point. This furrow is called "the primitive streak" (PS) (Fig. 23). The apex is called Hensen's Node (HN) [114,117].

A large part of the disk ingresses in the furrow, and starts to migrate underneath in opposite direction. The tissue that has ingressed forms the yolk-sac ${ }^{36}$ (YS), and other extra-embryonic organs, and also internal layers of the embryo (mesoderm essentially). At "some moment" the apex of the furrow starts to recede, and the embryo body will now truly be formed. Since the body features "appear" as Hensen's node recedes, it is believed that this structure (Hensen's node HN) generates the entire body plan. So that HN is called the embryo "organizer". In effect, while HN slowly moves southbound of the disk, bumps and folds appear on top of the disk which will form the neural plate, neural crest, eye rudiments, tail bud, limb buds, etc. (Fig. 24). A number of misconceptions have spread about the "organizers". In an historically important experiment Speeman and Mangold [118] transferred a frog organizer onto another blastula, and got almost mirror image embryos (two tadpoles co-joined by the belly). However, reinterpretation of the experiment shows apparently that if the organizer alone is transplanted, abnormal tissues are formed, without head nor tail. In order to get a more or less complete animal, a rather large patch of all three germ layers have to be transplanted, which renders this experiment almost meaningless (reviewed in Ref. [114]).

\footnotetext{
36 The yolk-sac is an organ which "digests" the yolk in order to feed the embryo. In the avian egg, it is the main extraembryonic organ. In mammals, the yolk-sac may become a placenta, or the placenta may be formed from any other extraembryonic organ. From the point of view of morphogenesis, the yolk-sac and placenta are actually parts of the embryo. The complex motions of gastrulation so to speak segregate the early mass of cells into a disc growing away (the yolk-sac) an folds converging towards the median axis (the embryo). How this is achieved is explained below. At birth, the remaining connection between the two is interrupted (navel).
}

The propagation of Hensen's Node across the presumptive embryo body is a deformation wave; it is not a translation of a physical object: HN is "made" of cells which come in and move out of it perpetually [116]. Hensen's Node "regenerates" itself spontaneously when it is excised [117]. The assays consisting in excising Hensen's node show clearly that the "regeneration" of Hensen's node is a progressive viscous closure (healing) of a large crack made in the embryo by the excision.

\subsection{Brief summary of cell movements during tetrapod formation}

If we follow from a physical point of view the embryo formation, it seems that the mechanism of animal formation is the following:

The animal is first round. It has retained from the cortical rotation a specific area located caudally, which has attracting properties. The disc contracts around this area and a soft "crack-like" furrow propagates like an involuting avalanche ${ }^{37}$, with a special region at the apex, similar to the apex of a crack (Hensen's node), and then recedes. By avalanche, it is meant that cells located at Hensen's Node, which start to dive and involute "downwards", (instead of crawling in plane) induce this same behaviour on the cells located somewhat ahead of the current position of Hensen's Node, but which are still in-plane. This is how the deformation wave, which Hensen's node actually is, propagates, giving the impression of a line of "infolding" which advances across the blastula, following a straight line which is akin to a soft crack (the correspondence with the crack is linked to the existence of force thresholds for the propagation kinetics: in a true crack above a threshold, the material ruptures, so the crack apex advances, here the cells contract and buckle inwards so that Hensen's node moves forward).

A large part of the embryo ingresses in this soft crack while it is open. The cells which have involuted and ingressed migrate away and form the yolk-sac. Then the

\footnotetext{
37 By this it is meant that a straight and double line of forward dive of the tissue propagates linearly from the presumptive tail to head.
} 
motion of the crack reverts, and cell ingression is now concomitant with crack recession and closure.

Crack recession and closure is followed immediately in space and time by embryo formation (folds). The folds which form truly the animal are formed from cells that keep on converging. Cells that migrate away are lost from the embryo, and form the extra-embryonic organs. The area of the contraction (the most contracted area) is unstable and generates the first vertebrate segments around the navel area, which trigger a segmentation wave across the embryo folds (the somites). As the folds located rostrally expand they remain open and become a head. The caudal fold closes up and becomes a tail, the closing of the fold is complete up to the cervical vertebrae. As the segmentation wave enters different areas of the folds, it generates first vertebrae then limbs and ribs, etc., by what might as well be the same segmentation process.

\subsection{Deformation and cell movements during tetrapod formation}

As it recedes, the line over which the crack ("Primitive Streak") was before, is folded into an embryo shape. As it finally arrives in the caudal region, Hensen's node progressively vanishes completely as if it were "healed", and new folds form above it which will be the caudal bud, and other caudal parts. While this entire process occurs, the initial disc is deformed into some sort of a regular "8-shape" or keyhole shape, centred on the presumptive navel. This 8 shape is in fact more slender and elongated in the median part, than far away [119].

The movements of the embryo may seem complex at first glance [120], and seem to require an exquisite biochemical balance, which may not be so. The cell flow and reorganisation seem to comprise different components, like passive advection [121], cell shape change and intercalation $[122,123]$, which are ascribed to different genetic pathways [119,124]; but these may as well be different outcomes of the same cell flow, the physical forces acting as epigenetic cues. Indeed, it is well known in foam (say soap bubbles) dynamics that foam walls may be advected or may reorganize, depending on local stress conditions [125]. Intercalation is equivalent to foam wall reorganization under compression, the cell tesselation being akin to a distribution of foam walls.

Cells seem first to flow towards the median axis with no apparent objective. Once the central fold starts to form, cells intercalate along the median axis. This is to say that, in addition to slowly drifting towards the median axis, they tend to rearrange to pile up in an orderly fashion along the presumptive backbone. Therefore, the formation of the embryo body seems a matter of a 2D flow, which becomes $3 \mathrm{D}$ by folding, and inside which cells reorganise to adopt regular textural orientations, i.e., the cells have a major axis of orientation, and cell-to-cell orientation correlates over considerable distances which parallel body plan directions, as obvious eventually in muscle, tendon, and cartilage orientations. The cell orientation is congruent with the macromolecular (mostly collagen fibers) orientation of the extra-cellular matrix, as observed directly in vivo by fluorescent techniques [126] (see also discussion of cartilage order in Sect. 9). Cells seem to simply align in the local stretch, as they are known to do in stretch forces, or in uniaxial shear [127,128].

The initial flow of cells exhibits large vortices [121,129-131], which imply that descriptions based on diffusion of chemicals are not correct, at least not complete. In particular, any organized early anteroposterior gradient dating from the ovocyte or the morula is advected and stirred in the pattern of eddies, therefore, there is nothing like a "pre-pattern" of the animal at the blastula stage, and in particular, the limb positions are certainly not fixed by early gradients of chemicals. Another problem lies in the fact that cells on the ectoderm and cells from the same pool which have invaginated inside the furrow (while Hensen's Node travels through the blastula), migrate in opposite directions, a fact which is explained currently by chemical gradients of different chemicals acting with opposite affinities: cells would be attracted by a chemo-attractant up to the median axis, then repelled by a chemorepellent [132]. The trouble is that cells are still attracted on one side, while others are already repelled on the other side. Many cells remain in the median area to form the body of the embryo. The fact that cells invaginate and migrate away renders the interpretation of molecular stainings extremely tricky. Many early stainings as seen for example in data base GEISHA (stages 1-5) are just impossible to interpret if one does not know that the stained cells correspond to both cells in the ectoderm moving in the distal-to-proximal direction and cells underneath in the ingressed mesoderm moving in the opposite direction, both following large vortices.

The cells which have ingressed and move away from the PS although they ingress along a line, will eventually form a perfectly circular piece of tissue (the yolk-sac), completely featureless (except for the vasculature, of course, Fig. 25), whose speed of growth is much larger by the edge than by the median axis, while the opposite would be expected for a chemorepellent diffusing away from the median axis (personal observation).

It is a priori stunning to see a piece of embryo, which has undergone invagination, migration, etc. form a completely circular, biological "body" larger than the embryo itself, devoid of any organ, and hence in a state of complete absence of morphogenetic instructions of any sort. From the point of view of logic, the yolk-sac is just the simplest animal, associated to a radiating topology: a round and stable vascularised piece of tissue.

In addition, the primitive streak, with $\mathrm{HN}$ at the apex, first grows in the posterior to anterior direction (in Fig. 23A, for example), then reverts to grow in an anterior to posterior direction (in Fig. 24 left). If HN is following chemical gradients, how can one explain the reversal of the gradient across the embryo body, in a very rapid time? [133] Not even mentioning that cells in Hensen's node are perpetually coming in and going out (it is a deformation wave). 

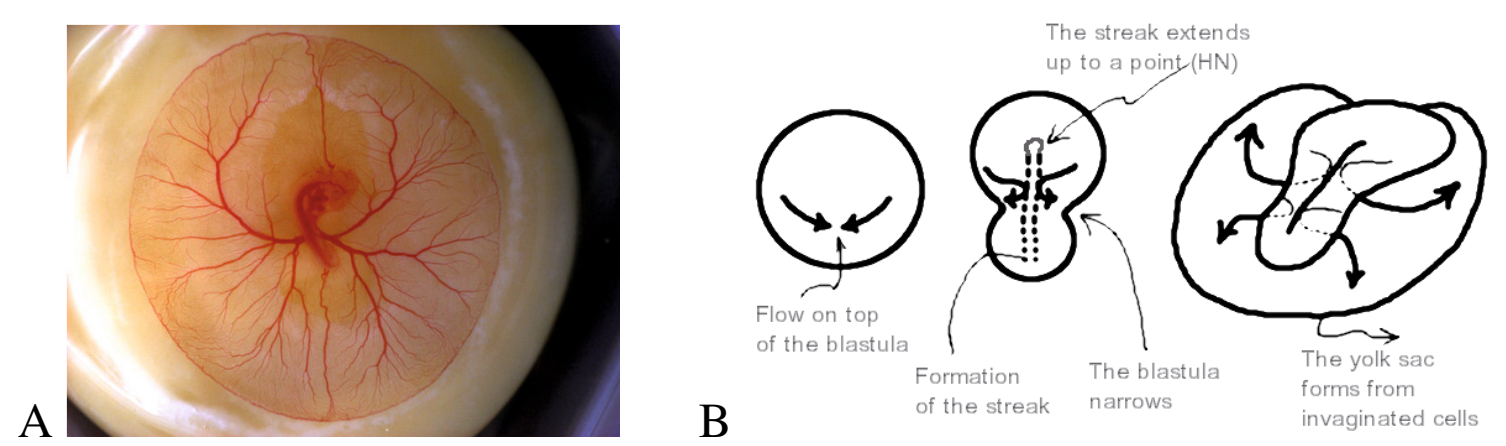

Fig. 25. (Color online) (A) A typical chicken embryo at 3 days of development showing the large featureless disk (the yolksac) formed by the cells which have ingressed into the primitive streak. In the centre, the embryo formed by the folds (From Ref. [151]). (B) Origin of the yolk-sac, explained schematically (let us insist that it is a continuous flow). Cells in the embryo (which are on top) and cells in the yolk-sac (which are underneath) migrate in opposite direction. Initially, cells converge towards the south of the embryo, then they invaginate, and move away. In Figure 25B right, the embryo finds itself in a state of "convergence" while the yolk-sac expands away.

\subsection{Significance of the hyperbolic point}

The early stages of vertebrate formation seem to progressively boil down to the following scenario. There exists a specific area in the bottom of the blastula, inherited from cortical rotation, and called Koller's ${ }^{38}$ sickle, which attracts neighboring cells ${ }^{39}$. The area which first attracts the cells has been shown to be marked by the gene nodal [134,135], and inhibition of nodal prevents formation of primitive streaks. Therefore the localized spot of this protein is candidate to be the early triggering event, although it may be only a remnant of some other previous event. As cells neighboring the nodal spot strive to converge towards this point, they generate a cellular flow which self-organizes into vortices ${ }^{40}$ (adding deformations and conformational changes to the problem). It has long been known that there were two large vortices inside the blastula disc [129], Figure 26.

However, for some epistemological reason, scholars neglected to observe that there were actually four revolu-

\footnotetext{
38 Or Rauber's sickle, depending on authors we shall now on call it KR sickle or KRS.

39 Cortical rotation is an entire subject in itself. Cortical rotation, as created by the impact of the spermatozoid, amounts to a shift of the outer shell of the cell, with respect to the inner cytosol by a rotation of magnitude $d \alpha$, if we assume that the ovocyte chemical content was at spatio-temporal equilibrium with respect to all cortical fluxes, cortical rotation creates an imbalance of fluxes proportional to $\operatorname{grad}(\mathrm{C}) . d \alpha$ of any concentration map. Therefore, one may wonder whether the attractive character of the embryo "organizers", may be a remote consequence of these imbalances trying to restore the earlier equilibrium, and hence provoking a global attraction towards the opposite point as the point of entry of the spermatozoid.

40 Stainings against $f g f 8$, wnt, nodal, etc. as displayed in the data base GEISHA (http://geisha.arizona.edu/geisha/) stage 3 of development, or in the original papers, clearly show that the dynamic pattern of these genes is an advection of an initial spot.
}

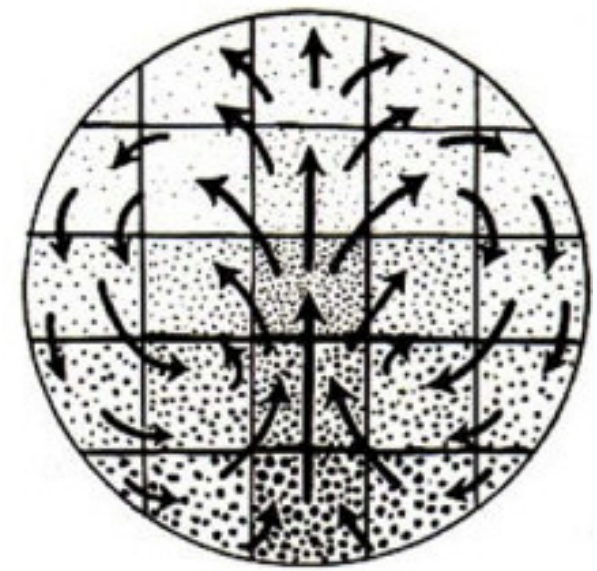

Fig. 26. The existence of vortices in the blastula has been known for long. Wetzel, R. Untersuchungen am Hühnchen. Die Entwicklung des Keims während der ersten beiden Bruttage. Arch. Entw. Mech. Org. 119, 188-321 (1929).

tions, and not two. Indeed, it is only recently that it has been realized that there exists a saddle-point, and that the cellular flows revolve away from this saddle-point in a hyperbolic fashion [121,131], as they collide in their march towards the nodal spot which is tself advected by the cellular flow (Fig. 27). While there exist two large and complete vortices spanning the main part of the blastula, "southbound" of the KRS, the cells revolve in the opposite direction, forming two other segments of vortices, difficult to image, especially considering that the flow is never quite in steady state, but which do exist.

This flow makes clear that a vector, or tensor field theory of development of vertebrates has to be put forward. The pattern of revolution shows the typical behaviour of a continuous material, be it a viscous or plastic material. In such a case, physical motion of one area, associated to a velocity $V$, implies a coordinated velocity in the 

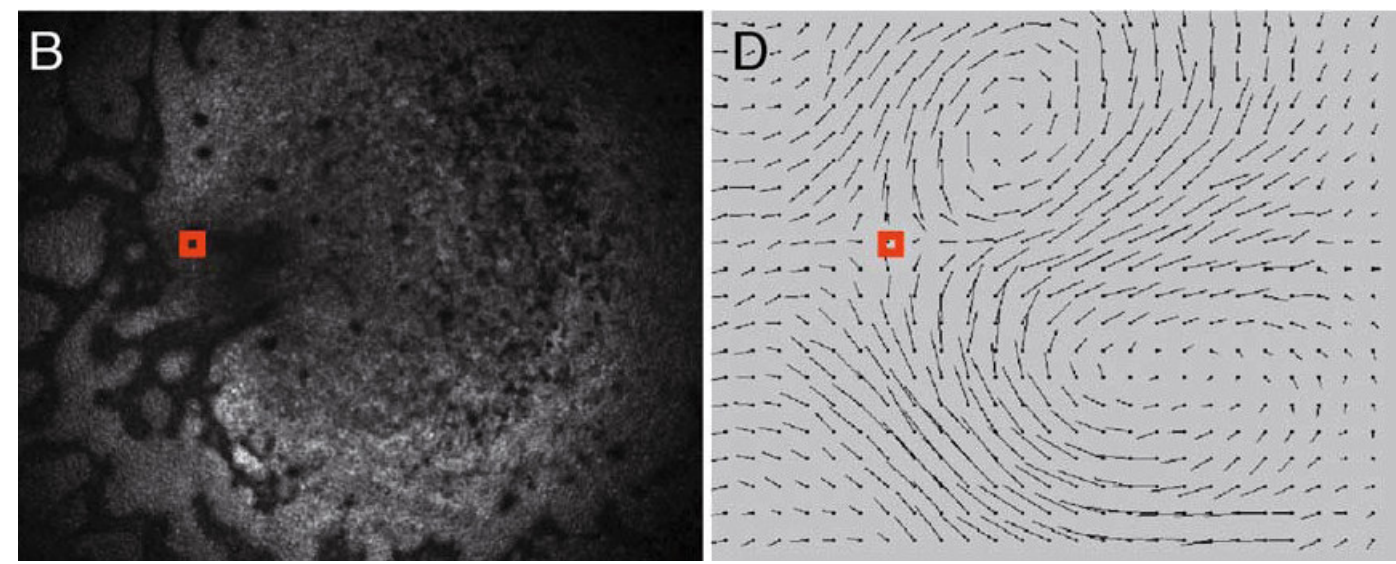

Fig. 27. (Color online) The vortices are now described by a "flow". These plates, from reference [121], show cell tracking during gastrulation movements in the chicken blastula. In the left plate, the blastula occupies almost the entire frame (3 mm). To the left of both plates, just below the red square, a furrow starts faintly to appear. The red square shows the position of the saddle-point, which coincides with the intersection of the KRS with the presumptive antero-posterior axis. The regularity and smoothness of the flow leads experts to consider it as hydrodynamic-like ([121] "cell flow patterns very much resemble flow patterns in a fluid, where viscosity controls the interactions and flow properties."). Right, tracking of velocity vectors (the nail representing the velocity vector has a length proportional to velocity modulus, it is oriented with the head upstream). These vectors correspond to the displacement over $40 \mathrm{~min}$ of cells. The magnitude of the velocities are typically of 0.5 microns per second.

neighbourhood, by mass conservation law. If the material is assumed incompressible ${ }^{41}$, the conservation of matter at each point implies a differential relationship of the form $\operatorname{div}(V)=0$, which relates spatially the motions. Stated otherwise, a single cell moving across the material has to move apart the material ahead of it, in order to move forward. This creates an interaction between each cell and it surroundings, that can be treated by continuum mechanics theory.

Especially, in the present case where the blastula material is seen to form a saddle-point, it should be insisted that it is very difficult to generate and stabilize a saddlepoint with only chemotactism towards chemical gradients, because potential flows (of the form $\operatorname{grad}(C)$ ) do not admit circular trajectories in the absence of discontinuities. Stated otherwise, when a loop is observed in the cellular traffic, and if one assumes that cells go up concentration gradients, then following the loop, one returns at the same point after completion of the loop, although this point is now supposed to be located at a higher concentration value since the gradient was followed constantly uphill. In order to generate loops one needs (at least) two sources, one positive and one negative, such that a dipolar flow is generated. In this case, the discontinuity occurs across the dipole, when jumping from the negative source to the positive one, while the field far away has a pattern of loops. Conversely, vortices are classical solutions of physical vector fields, such as hydrodynamic flows, and are easily obtained as a consequence of volume forces.

A side result of this analysis is that the physical difference between arthropods and vertebrates is that say, the gradient of bicoid in arthropods is static, (as it generates the segments), while the gradient of nodal in vertebrates induces immediately a flow which patterns the

\footnotetext{
41 Blastulas are composed of $95 \%$ water.
}

embryo with a chord, limb fields, etc. prior to segmentation. Intuitively, it is clear that time shifts between the segmentation process and the movements may be a cause of animal evolution [135].

\section{Simple mathematical integration of movements of interacting cells}

\subsection{The cell traction field}

We now progressively proceed to the physics of the problem. Two complementary views of the problem exist at present. Yang and co-workers have proposed as hypothesis that the cellular flows were generated by pools of FGF8 and FGF4 acting as negative and positive feedbacks on cell migration [132]. However, it is not clear whether the concentration levels used to asses chemotactic properties are related to physiological concentrations at the moment of initial cell flow. In addition, while there does exist a pool of FGF8 in the area of the saddle point, at start of the flow, many other molecules have the same initial concentration map (see below), and they follow the same dynamics, as the flow itself advects the pools of chemicals [132]: when the FGF8 pattern is followed dynamically, it seems to be merely advected by the flow, and not to create it, this is also true of many other genetic expressions. In addition there is a logical inconsistency in ascribing the flow to static chemical gradients. However, the observation of a saddle-point implies the existence of at least 4 such pools, and not two, and these are not present initially, to our knowledge.

The other view is that the movement of the cells is intrinsically dipolar, and, taking into account the initial condition, the flow will be trapped in a generic movement, with self-organizing properties. In this view, the 
low Reynolds blastula flow, acting on an initial symmetry breaking, scales up the "cross pattern" latent in the blastula, and inherited from early cell divisions. This scaling up is an instance of the principle of Curie, by which the final pattern formed by a dynamic process, reflects the broken symmetry of the initial conditions. The flow, with its typical vortices, is an emergent property of the field equations, and is not anywhere "coded" as such.

The saddle-point, as observed in the blastulas, is associated to a hyperbolic flow, and it can be obtained by the collision head on of 2 jet-flows [131], parallel to the KRS, but with opposite orientations, which would have the cortical rotation line (line of first cleavage), and the sperm entry point (second cleavage line) as remote origin. By generating a pattern of cell walls in the form of a cross, the initial cell divisions induce a preferred path (the integral of filopodia forces is biased); further durotaxis would orient cell traction forces in a pattern having the same symmetry, which would itself be scaled up by the flow field into a dipolar flow. Each jet requires one dipole of flow, and the saddle-point is actually a quadrupolar flow, and not a dipolar flow.

Since a vortex flow has obviously to be invoked. Cui and co-workers suggested a Rayleigh-Bénard ${ }^{42}$ type vortex flow induced by a stable gradient of polarising molecules [121], however it has not been shown at a technical level whether this possibility stands. The authors underline the importance of hydrodynamics in the establishment of the flow. One cannot simply consider that cells follow chemical gradients with a speed $v=$ $\operatorname{grad} C 1+\operatorname{grad} C 2+\operatorname{grad} C 3+\operatorname{grad} C 4$, because this form of motion does not comply with Newton's law. In effect, Newton's law fixes relationships between forces and accelerations. If one writes ex nihilo a relationship of the form $v=\operatorname{grad} C$ of cells moving inside a blastula, one then assumes implicitly a viscous behaviour, because only the force of cells may be related to $\operatorname{grad} C$, not the absolute velocity. However, in such a case, a viscous behaviour implies drag on the surrounding material, which is absent from the ansatz $v=\operatorname{grad} C$. Such an ansatz does not treat the advection of the pools of concentration by the bulk flow, and it does not treat the interaction between cells. It has been observed that the original pool of FGF8 is advected by the flow [134], and also that when an extra pool of FGF8 is positioned in the yolk-sac, cell motion is biased towards the pool of FGF8, but the entire pool of FGF8 is itself advected away by the cell flow ([132], Fig. 5D), which is typical of a viscous material flowing away under the action of a volume force (otherwise one would expect a convergent flow toward a static pool).

However, the existence of a saddle-point implies that the vortices are not vortices corresponding to the onset of a R-B-like instability. In R-B instability, a steady pattern

\footnotetext{
${ }^{42}$ Rayleigh-Bénard instability is observed when a liquid confined between two plates is heated from below. As the warm liquid rises, competition between viscous effects and buoyancy generates a pattern of rolls. In the Rayleigh-Bénard instability, the flow revolves from bottom to top, and back. The fluid speed is zero at the plates surface, and the plates are static.
}

of eddies forms which revolve from one end of the gradient of temperature to the other end (the cold liquid falls down towards the warm plate, and then raises). The existence of a saddle point inside the blastula would imply quite non-linear vortices which is unlikely (no spontaneous symmetry breaking at such low Reynolds), or two R-B cells rocked at $90^{\circ}$ and facing each other. In addition, the form of the embryo is a matter of transport into the flow of the pattern itself, while in R-B convection, the boundaries are immobile. A way to understand this approach consists in considering the FGFs pools as dynamically driving the boundary of the blastula during the revolution. Numerical solutions based on large scale computation of cellular behaviours are starting to emerge $[137,138]$, however, when dealing with very large number of cells, a continuum mechanics approach becomes possible.

In this spirit, a simple hydrodynamic analysis of the problem was put forward in reference [131]. In a different approach I have proposed to treat directly the influence of mechanics and of cell-to-cell interaction, in this problem. It is getting increasingly evident that mechanical factors play an important role in embryo development [139]. The recent progress should not shadow the remarkable pioneering work of Jacobson and Gordon [140], who were able already in 1976 to produce simple numerical simulations of convergent extension ("morphodynamics") of embryo body based on only a few cell behaviours, such as shrinkage and extension, although several aspects are not accurate due to absence of ingression in the model, to the fact that the notochord is put by hand, and that dipoles are not introduced in the model. It seems to the author, that the mechanical approach to embryo development has been overtaken by genetics, probably because tensorial analysis is intrinsically difficult, and because it was believed that biology followed specific laws, almost beyond physics; there is no a priori reason to assume that shapes form by following static gradients of scalars, building up fields of "information position".

Physics plays a role in development through several aspects: first of all any push, swelling, fold, etc. that may be observed in biology has to comply with Newton's law. In a given material, a constitutive equation relates stresses to deformations or deformation rates so that biochemistry may already play an important role by modifying viscoelastic parameters of the living material (for example making it more or less viscous), or by modifying the kind of physical behaviour followed by the material (for example, a given material may be homogeneous, and follow a hydrodynamic-like equation, or be oriented, and follow a liquid-crystal type of equation).

It has been shown recently, in many instances, that genetic expressions are induced by mechanical forces [23,141-144], and even that maps of genetic inductions may in some if not all cases, be viewed as stress maps [142]. This is especially true of FGF10 map. Also, mechanics orients actually the cellular division [145], modifies and is modified by cell adhesion [146], may regulate the interstitial volume of water $[147,148]$, thus regulating the effect of any soluble growth factor. 


\section{Fleury: Clarifying tetrapod embryogenesis}

In addition, gradients of material properties induce morphogenetic gradients, either by modifying chemotaxis ("durotaxis" $[149,150]$ ), or by inducing material flows [151]. If one believes that chemistry controls mechanical behaviours, then in return, mechanical forces might have an effect on chemistry (in general terms, stresses are always a component of reaction kinetics and affinities).

There is ample evidence that the early embryo material is very close to a viscous material [152], at early stages. In addition, at such early stages, embryos are composed of thin cellular sheets [111]. Therefore, there is reasonable hope that embryo development can be understood by a hydrodynamic analysis in terms of flow, at least for the early stages, when the movement is in plane and the embryo very fluid. However, after a phase of in plane flow, folds appear which require to treat the out-of-plane buckling, with the proper source terms of stress.

Newton's law requires writing down the equilibrium of the stresses, against a given force field, for example gravity, or any other volume force exerted by the material. In the case of living material, there exist obviously forces exerted by cells. Typical forces exerted by cells are force monopoles (swellings), as in plants, and force dipoles (traction forces), as in animals, although animal cells may also exert monopoles. Dipolar forces are produced via extension and contraction of fillopodia or lamellipodia, or by cilliary propulsion. Separating the monopolar and dipolar source terms is a classical approach in hydrodynamics, for example the Papkovich-Neuber [153] formulation of the Stokes equation.

If we assume a volume force f, writing Newton's law for slow creeping flows (the blastula revolves by approximately $4 \mathrm{~mm}$, in about $10 \mathrm{~h}$ ), gives Stokes equation, which will write, with $\mathbf{v}$ the deformation rate vector, $P$ the pressure:

$$
\nu \Delta \mathbf{v}-\operatorname{grad} P=f .
$$

In a recent article [131], it was shown that the rate-ofdeformation field imparted by a single cell pulling in a thin sheet blastula of thickness $h$ with a force $f$ can be reduced to a vortex dipole given by:

$$
\mathbf{v}=\left(-f h^{2} / 12 \nu\right) \operatorname{curl}(\Psi)
$$

in which $h$ is the blastula thickness, $\nu$ an equivalent viscosity and $\Psi$ is the scalar stream function ( $z$-component of the vector potential [154]),

$$
\Psi(x, y)=x /\left(x^{2}+y^{2}\right) .
$$

Which gives the flow map given in Figure 28 (the image shows iso- $\Psi$ lines, which are the stream lines). This analytic expression is found assuming that the viscous component of the blastula behaves like a newtonian fluid. However, it is also shown that the elastic component has the same shape if the material is incompressible (this is because in thin shells, the perpendicular component of the deformation gradient is the strongest, therefore a lubrication approximation can be performed for the deformation field).

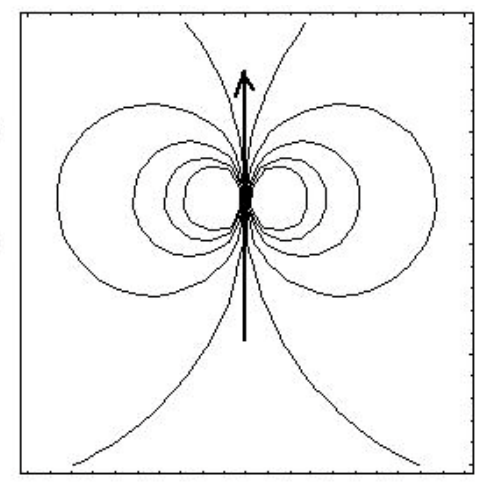

Fig. 28. Flow map induced by a single cell in a viscous sheet, in dimensionless form. The flow is dimensioned by a factor $f h^{2} / 12 \nu$ (Eq. (2)).

This shows that a localized increment of force of a given cell tends spontaneously to generate whirls even in the absence of complex genetic pools (this is known as the "dipolar field"). Although a pure hydrodynamic equation was used, similar patterns, at qualitative level, may be obtained with other material behaviours.

One important observation about this flow is that mass conservation is achieved around the cell by pushing forward and recirculating in the back, the material ahead. This explains intuitively the origin of vortices in blastulas. There is no need to generate a pattern of stable vortices by chemical gradients (which is extremely difficult), to induce circular streamlines which tend to self-organize. In thin shells, the push of cells induces vortices by the effect of physical laws. In addition, this shows why the usual writing $v=\operatorname{grad}(C)$ of chemotactism is questionable. In fact, such a writing assumes a friction-less medium, across which cells can navigate without hindrance, which is certainly not the case, especially, the epiblast is a continuum material of connected cells. Writing down the true equations of morphogenesis requires solving for the entire deformation field around a single cell, which amounts to a long ranged cell-cell interaction, and next the deformation field for all cells. This amounts to the integral of dipoledipole interactions between cells, as already predicted on theoretical grounds [155].

When many cells exert a force, the flow is obtained by integrating the dipole formula over the cellular distribution of forces, because the slow flow equation is additive.

\subsection{Examples of typical flows generated by cell configurations}

Example 1: From equation (3) it follows that a line of cells located between positions labeled a and $-\mathrm{a}$, each one exerting a force $\mathrm{f}$ can be obtained by the integral:

$$
\begin{aligned}
\chi_{1}(x, y) & =\int_{-a}^{+a}(y) /\left((x-\alpha)^{2}+(y-b)^{2}\right) d \alpha \\
\Psi_{1}(x, y, a) & =\operatorname{ArgTan}[(x-a) / y]-\operatorname{ArgTan}[(x+a) / y] .
\end{aligned}
$$



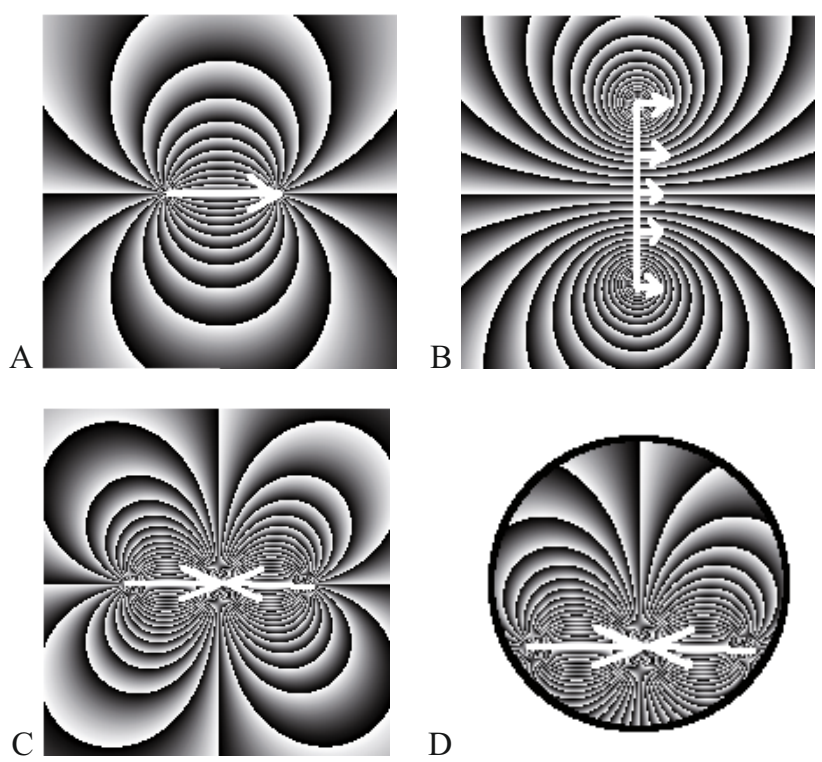

$\mathrm{D}$

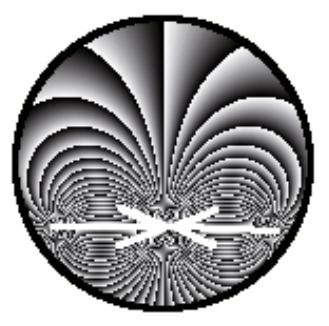

Fig. 29. (A) The flow map exerted by a line of cells aligned along the same line, each one pulling with the same force (here $b=0$, and $a=1 / 6)$. The figure shows the levels of the stream function $\Psi$ (with a linear shaded grayscale between levels, in dimensionless form. The actual scale is defined by the viscosity of the material, the magnitude of the pulling force, and the blastula thickness). The stream function iso-values curves are locally tangent to $v$. (B) The flow map exerted by a segment of cells, each one pulling parallel to the horizontal-axis, but themselves piled up along the vertical axis, with the same force. (C) The flow map exerted by two segments of cells exerting a contraction towards a hyperbolic point. (D) The flow map as it should be viewed on the blastula at the beginning of gastrulation.

Which gives the flow map given in the Figure 29A.

Example 2: from equation (3) it follows that a segment of parallel cells pulling forward exerts a force obtained by the integral:

$$
\begin{aligned}
\Psi_{2}(x, y, c)= & \int_{-c}^{+c}(y-\alpha) /\left((x-a)^{2}+(y-\alpha)^{2}\right) d \alpha \\
& \left.=(1 / 2) \log \left(\left(x^{2}+(y-c)^{2}\right) / x^{2}+y^{2}\right)\right) \\
& \left.-(1 / 2) \log \left(\left(x^{2}+(y+c)^{2}\right) / x^{2}+y^{2}\right)\right)
\end{aligned}
$$

Which gives the flow map given in Figure 29B.

Another solution exists, which is a solid body revolution around a point. Indeed, a straightforward calculation shows that the stream function for a uniform volume force oriented along the orthoradial vector, in polar coordinates, gives a circular vortex with an orthoradial speed which varies linearly with the distance to the center, like a merry-go-round (solid-body revolution). This type of revolving vortex is observed for cilliary flows of epithelial cells (Roberto Camassa, personal communication), when the epithelial cells are attached bio-chemically to a substrate. In this case, the epithelial cells are immobile, and the culture medium above them revolves with a viscous vortex which has a solid-body revolution pattern.

\section{Application to embryo morphogenesis}

\subsection{Simplification of generic embryo movements}

The movements observed during gastrulation may look very complex, and this is why the role of many molecular expressions are investigated. At present, the back and forth motions of cells in Hensen's node, and the back and forth motion of cells towards the primitive streak and away, are "explained" by chemical gradients acting one way, or the other way around, at given times. It is however not certain that it is necessary to invoke first the existence of a chemo-attractant along the body axis for cells of the epiblast, in order to explain their motion towards the AP axis, and then a chemo-repellent, which presumably starts to act as soon as the cells dive forward, in order to explain why the mesoderm migrates away from the AP axis, immediately after having migrated towards it. One important unsolved question is how evolution could have selected independently a chemical process of opening of a streak and another chemical process for closing of the same streak, while forming for supposedly many generations a viable animal with an open streak. It is more logical that the two phenomena are correlated enough, that in some range of parameters, they will occur "automatically" i.e. the opening is necessarily followed by closure.

Since the entire geometry of the blastula and the boundary conditions of the cellular flows change as the primitive streak opens and cells invaginate, one must also address the impact of these phenomena on the directions of the flows. One finds a reasonable explanation of gastrulation movements, when taking into account the change in boundary conditions during the cellular flows.

As stated above (Sect. 5), gastrulation starts by the pulling action inside a sector of cells $[122,130,156]$. The cells may have been prepared in this state by earlier events, what matters is that they start off with a specific orientation. The cells may actually flow, in which case they exert a pulling action, or they may change shape, in which case they exert a small quadrupole (they pull in one direction, and push in the other). It makes little difference, from the point of view of physics, and at this level of description, because of linearity of the solutions, with respect to the sources of force.

Since the initial cortical rotation is a physical process that creates a mismatch of all chemicals between the cytosol and the cortex, and since the area of KRS is very thin, we may assume that the cells are all identical inside this sector of cells, and find the flow map inside the epiblast, by positioning two solutions of equation (4) head to head (Figs. 29C, 29D). The corresponding stream function is:

$$
\begin{aligned}
\Psi_{3}= & \operatorname{ArcTan}[(x-a) /(y)]-\operatorname{ArcTan}[(x) /(y)] \\
& -(\operatorname{ArcTan}[(x) /(y)]-\operatorname{ArcTan}[(x+a) /(y)]) .
\end{aligned}
$$

This implies the existence of a high stress point located at the edge of the segment and advected towards the crossing of the sector and the $\mathrm{AP}$ axis, this point is apparent 
in Figures 29C,29D as the hyperbolic point (flow is convergent in one direction, divergent in the other). More precisely, the flow exhibits a hyperbolic pattern around the neutral point (saddle-point). This is associated to hyperbolic lines which correspond to stream function of the form $\Psi(x, y)=k x y$ ( $\Psi=$ cte defines the streamlines). Around this point, the hyperbolic flow can be linearized such that the $x$ and $y$ components write:

$$
\begin{aligned}
& v_{x}=-k x \\
& v_{y}=k y
\end{aligned}
$$

with $k$ a dimensioning constant. The velocities are linear. In case of an inertial flow, the pressure (stress) is found by Bernouilli's law, by writing $P=P_{0} / \rho-v^{2} / 2$ which gives a maximum pressure at the saddle point $(\mathbf{v}=(0,0))$. For a viscous flow following a Poiseuille profile $\mathbf{v}=-h^{2} / 12 \nu \operatorname{grad}(P)$, where $h$ is the layer thickness and $\nu$ the viscosity; the solution for the pressure is given by

$$
P=P_{0}+\left(6 \nu / h^{2}\right)\left(k x^{2}-k y^{2}\right) .
$$

Which shows that the flow arrives along the $\mathrm{x}$ axis from a region of high stress (high $x^{2}$ ), and flows away in the y direction towards regions of low stress $\left(l o w-y^{2}\right)$. Therefore, the area around the saddle point is at high stress, and the stress decreases in a saddle form away from it anteriorly, and posteriorly.

It is known that polarised cell flow can be induced by a mechanical stress [163]. Therefore if there exists a threshold of stress such that cells contract to invaginate (some sort of escape of the stress), this threshold will first be reached at the most compressed point. From there, cells will start to invaginate in an avalanche mechanism, and a line of epiboly will propagate like a crack towards the caudal pole and the anterior pole, as observed. This crack is what was described above as the primitive streak (PS), Figure 23. However, since the pulling sector is already caudal, the propagation is mostly conspicuous in the anterior direction, starting from the posterior hyperbolic point (Fig. 23 left). It has been stated for long that the primitive streak propagates in the posterior to anterior direction [111]. In my opinion, this is actually wrong (personal observation). The primitive streak propagates in both directions, from the intersection of Koler-Rauber's sickle with the median axis.

Now, as the PS propagates, it enters regions where the stress in the tissue is smaller, because of the recirculation of the flow (the term $-k y^{2}$ in equation (8) brings a negative component).

As a consequence, the streak will propagate up to a point where the sum of the contractile stress exerted by the PS, and the surrounding stress linked to the KRS add up to a stress which finds itself below the threshold for epiboly. There, the furrow should end, and this is the point of arrest of Hensen's node (HN). The Primitive Streak has a length $L(\mathrm{PS})$ at which the stress term exerted by the cells along the PS cancels the stress created by the KRS. Quantitatively, one can estimate this distance since knowing $f 1$ the individual force exerted by cells inside the contractile sector, and $f 2$ the force of the epibolizing cells, the distance is such that $L(\mathrm{PS})=L(\mathrm{KRS}) f 1 / f 2$. There is little doubt that the pulling or contracting force of cells should be of comparable magnitudes, so that the length of the primitive streak is of the same order of magnitude as that of the KRS, hence the body axis occupies a large portion of the blastula.

Now, as cells ingress inside the (presumptive) embryo, the traction force at the epiboly adds a flow component along the furrow which is given by equation (5), with the corresponding length $c=L(\mathrm{PS}) / 2$. Indeed, we assume that cells which start to epibolize exert a traction on the epiblast fixed by internal dynamics, and independent of possible gradients. This amounts to supposing that cells are in a bistable state: either they fold inwards or they don't, but if they do, they do so with equal force, simply because the cell genetic content is supposedly the same. Therefore, when the primitive streak exists, the flow field is as in equation (5), and the cells progressively invaginate along the streak, as schematized in Figure 25B right. However, as cells invaginate, make a U turn, and start to migrate in opposite direction, they form the yolk-sac (YS).

Cells in the YS migrate away from the streak, underneath with a similar flow as equation (4). It has been shown recently that the flow of cells in a field like equation (4), generates automatically a round YS around the embryo (exact solution of the cell transport) when no specific morphogenic instruction of any sort is assumed [119].

\subsection{Coupling chemical gradients, mechanotransduction, and hydrodynamics}

It is not established whether the flow pattern organizes itself in response to chemical gradients present in the entire blastula, which fine tune the vector field at each spot. At present, the most reasonable origin of the entire movement, is the force term generated inside this narrow area (Koller's sickle) [157]. Which in turn, and together with stress forces, induces such growth factors as FGF8, and transcription factors as Brachiury (Tbox). Once "triggered", the flow continues and self-organizes. However, pools of chemicals do exist later during embryo movements, which have chemotactic properties, and the entire morphogenesis may become a complex mix of chemotactism and physical flow, with possible gradients of cell adhesiveness. Biologists sometimes attempt to distinguish bulk flow and cell migration, although bulk migration is induced by cell force (hence individual migration) [158]. Moreover, this is done by separating the motion of the extra-cellular matrix from the motion of the cells. It must be observed that both form a physical layer but only the moving cells exert forces. Therefore, by the principle of action and reaction, the extra-cellular matrix bears an opposite force as that of cells, therefore both the motion of cells, and the antagonist motion of the ECM have a hydrodynamic-like "bulk" component, and one cannot state that the ECM flow is the bulk flow and the chemotactism the difference between the total flow and the extracellular matrix flow. 

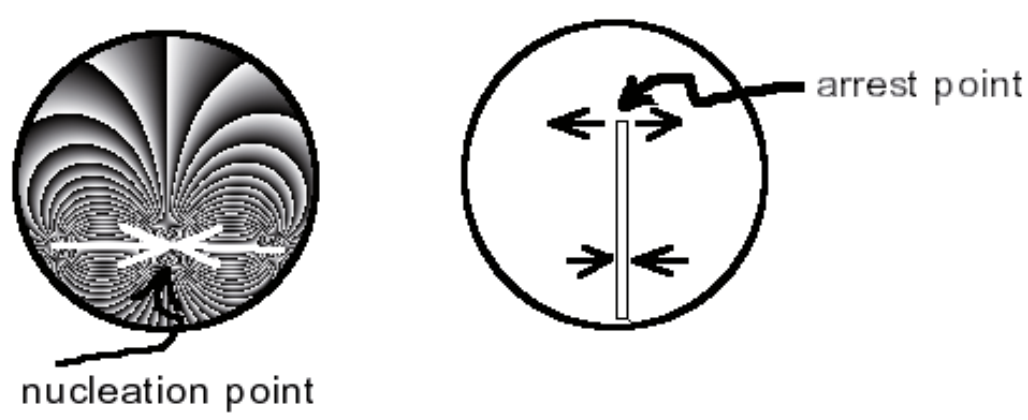

Fig. 30. While the furrow nucleates at the most stressed point it will stop when the stress reverts below the threshold for epiboly. This will occur as the furrow enters regions of opposite stress in the anterior part. In the figure above, right, we show the stress state in the region of nucleation of the streak, and the stress state, in the region of arrest. The lines represent stream lines. The flow is organized from a high pressure point located in the area of the presumptive navel, towards a low pressure point located in the anterior area.

The weakness of the chemotactic hypothesis is that it requires a fine tuning of molecular pathways, and an explanation of the origin of each pool of chemicals, spatiotemporally, which is still lacking, not even mentioning the phylogeny of the entire flow pattern.

This is to say that the inductive view finds itself in a never ending regression, trying to find a prepattern, which actually is permanently advected by the flow (what biologists call "translocation" or "dynamic expression"). This hypothesis considers the fine tuning of all elements in the problem as crucial to the appearance of the animals, up to the point that it is generally believed that animals could as well not have appeared, and that random events played a great role. I believe this is a misleading view. If animals form from an active material which produces under stress the growth factors congruently with the morphogenetic field, the fine tuning of the chemistry is less essential: many aspects are absorbed into scaling parameters.

The problem with the hydrodynamic hypothesis is that it seems uncorrelated to the presence of chemicals, except for the initial condition, although it is well known that the morphogenesis can be completely disrupted by brute force administration of many chemicals $[159,160]$. One interest of the hydrodynamic hypothesis is that direct inspection of cellular flows show that the morphogenesis of the embryo pattern is qualitatively complete after less than one cellular revolution. This is to say that, contrary to common knowledge, global morphogenesis is very rapid (between one and two days in the chicken), and does not require many iterations of stop-and-go discrete inductions ${ }^{43}$ (basically: a horizontal bar is advected and flattened to become a vertical one, the process requires $1 / 4$ th of a revolution).

A way of reconciling the two approaches consists in writing the constitutive equation as (assuming that cells

\footnotetext{
43 Anecdotally, the best physical analogue of formation of a vertebrate embryo is observed during Chinese shrimp chip frying. Chinese shrimp chips are delivered with a saddle point. When fried, they undergo considerable deformation. The form obtained transiently is very much similar to an embryo at the gastrula stage, just after formation of the neural crest.
}

align in the main shear force)

$$
\nu \Delta \mathbf{v}-\operatorname{grad} P=f \mathbf{v} /\|\mathbf{v}\| .
$$

And add the chemotactants as volume forces of the form $\operatorname{grad}(C)$. However, it has been shown that many growth factors especially that of the FGF family are in fact regulated linearly by pressure [142]. In this case, $\operatorname{grad}(C)$ can be written as proportional to $\operatorname{grad}(P)$.

$$
\operatorname{grad}(C)=\alpha \operatorname{grad}(P)
$$

and the constitutive equation becomes of the form:

$$
\nu \Delta \mathbf{v}-(1+\alpha) \operatorname{grad} P=f \mathbf{v} /\|\mathbf{v}\| .
$$

This allows to couple the chemotactism and the volume force exerted by cells, into an equation of the Stokes form with rescaled parameters. This is a closed equation. It provides a model system, in which the chemotactants are produced dynamically by the flow itself. In this view, cells generate by stress the growth factors which are their own incentive for the continuation of their movement, and cells use the established pattern to generate the orientation of the physical force. This situation is known to occur in lung or kidney development, and it is known that FGF are crucial growth factors for both limbs and lungs [52], and this is also true of Tbx 5 for lungs ${ }^{44}[161]$. Therefore, if the behaviour of $f g f$ 's with respect to force is the same in limb mesoderm, and in lung mesoderm, then an equation like equation (11) provides a simple constitutive equation for the viscous living sheet, assuming that biochemical production is in steady state with respect to the flow. As a consequence, the overall apparent motion of cells following growth factors and pulling themselves forward in the

${ }^{44}$ One should observe that FGF10, Tbx4 and Tbx5 are all important in limb and lung development. However, overexpression of Tbx5 in the lung has a similar effect as an increase in pressure (acceleration of branch growth). It may be possible that the same pathways are used in limb and lung development, although the topological difference between limb and lung is that the order of lung cell sheets is "inside out" as compared to a limb. 
direction of the flow, is a Stokes flow of the cells in a less viscous material, but with the same force oriented along the local existing streamline. Although the true constitutive equation might be formally more complex, it does not render the phenomenon itself more complex.

As explained above, the flow is a quadrupolar hyperbolic flow. It has been shown that such a flow transforms the blastula, and everything inside, into elongated "8" shapes $[16,131]$. This explains very well the overall form of the early embryo, and also, of several pools of chemicals, whose form can be understood on the basis of a lagrangian description of the flow (see Sect. 7). Especially, stainings against FGF8, which is so important for development, at early stages, have shown that there exists a localized spot of FGF8 around the contraction centre, which progressively becomes a slender 8-shaped zone of expression [162], Figure 31. (See also other gene stainings, especially wnt, in GEISHA database, or in Ref. [162]).

This fits perfectly well with a biomechanical advection in a hyperbolic flow, and shows how chemical gradients are reshuffled by the stream. This is actually permanently read in developmental articles, where genetic expressions are constantly described as "dynamic" (passim). A static diffusible chemical/inductive explanation requires a formidable analysis of chemical reactions, to explain the dynamical change of a circular spot into an elongated bar or an elongated "8-shape".

This field of research will probably progress by finding a more realistic equation for the chemotactic fields (if any), or by numerical integration of advection-diffusion equations for the growth factors. It is important in this context to measure locally the mechanical properties of the tissue, and correlate genetic expressions dynamically, to the stress fields. From the point of view of regenerative medicine, it is important to know whether the proper boundary conditions for a limb bud could be artificially reproduced by some artefact. While it is unlikely that an entire limb or hand could be properly generated (the joint organization will not be fit), it is reasonable to imagine that an isolated limb paddle of arbitrary length could be reconstructed, to taylor at will rows of digital rays, or even rows of fingers.

\subsection{Significance of the closure of the primitive streak}

As the YS expands away, it adds a stress which contributes negatively to the stress of the PS and the stress of the KRS. Therefore, the primitive streak, which is an open furrow of epiboly, progressively recedes, as increases the total stress the cells (located at the epiboly line) have to fight to succeed to invaginate. Stated otherwise, the flow linked to the KRS, plus the flow linked to the YS progressively shuts the primitive streak like a zipper, as observed. At the apex of the zipper, the stress is equal to the threshold for cell epiboly. Therefore, Hensen's node indeed moves forward during the "crack opening" and then recedes during the "crack closure", but this back and forth motion of the HN might simply correspond, as observed, to a deformation wave in the area of the apex of the crack, controlled by the genetics of cell traction force.

Now, as the PS closes, cells which move towards the AP axis start to intercalate. This word, in biology, means the deformation of cells by which they move their neighbourcells sideways to intercalate between them. Although there may exist chemical incentives to intercalation [120,166], a natural explanation is that cells moving towards the AP axis, as they do, under the action of the epiboly traction, will intercalate if they are hindered in their epiboly motion. In simple words, intercalation would be just the same thing as an epiboly or a cell migration, it depends only on the stress context (epigenetic cue) of the given cells which try to move forward. On mathematical grounds, an intercalation along a line such as the AP line is given by the mathematical solution of equation (4) (rocked to the vertical, and with a minus sign), if we assume that cells at the intercalation line all behave identically, which is a crude assumption, however, this assumption is justified by the fact that the hyperbolic flow acts as a massive stretch along the median axis of the initial spot of FGF8, therefore, we may expect the possible inhomogeneities to be smoothed away.

In other words: intercalation of a cell causes an increment of stretch or extension along the AP axis, as observed, and the integral of these individual stretches can be modelled by a flow. The parallel intercalation of all cells generates a large quadrupole along the body, centred on the neutral point of stretch (presumptive navel).

So, along the body axis where the primitive streak is already closed, the tissue feels a deformation field which is the sum of a stress due to cellular divergence from the PS which tends to orient the flow away from the PS, and an extension flow along the body axis oriented away from the navel due to cellular intercalation. This flow pattern writes (again with the assumptions that cells along the midline behave mechanically in the same way):

$$
\begin{aligned}
\Psi_{1}(x, y)+\Psi_{2}(x, y) & =\alpha\left\{(1 / 2)\left[\log \left(\left(x^{2}+(y-c)^{2}\right) / x^{2}+y^{2}\right)\right)\right. \\
& \left.\left.\left.-(1 / 2) \log \left(\left(x^{2}+(y+d)^{2}\right) / x^{2}+y^{2}\right)\right)\right]\right\} \\
& +\beta\{\operatorname{Arg} \operatorname{Tan}[(x-f) /(y)] \\
& -2 \operatorname{ArgTan}[x / y]+\operatorname{Arg} \operatorname{Tan}[(x+g) /(y)]\} .
\end{aligned}
$$

Or, in a more pictorial representation (Fig. 32):

where $\alpha$ and $\beta$ are ad hoc parameters, of comparable values related to the magnitude of the force terms, and possible gradients, controlled of course by genetic data. Because of symmetry around the initial nucleation point, the flows are centred around the presumptive navel, where a singular point exists. $c, d$ are parameters of the contraction segment and $f, g$ parameters of the extension segment, characterizing the length of the streak, and of the epiboly line. We assume that these are in proportion of the cellular forces, and the number of pulling cells.

Now, such figures as the embryo shape represented in Figures 23, 24 become quite clear. As the stress on top of the epiblast is released, the primitive streak recedes (crack closure). However, as more cells intercalate, the 

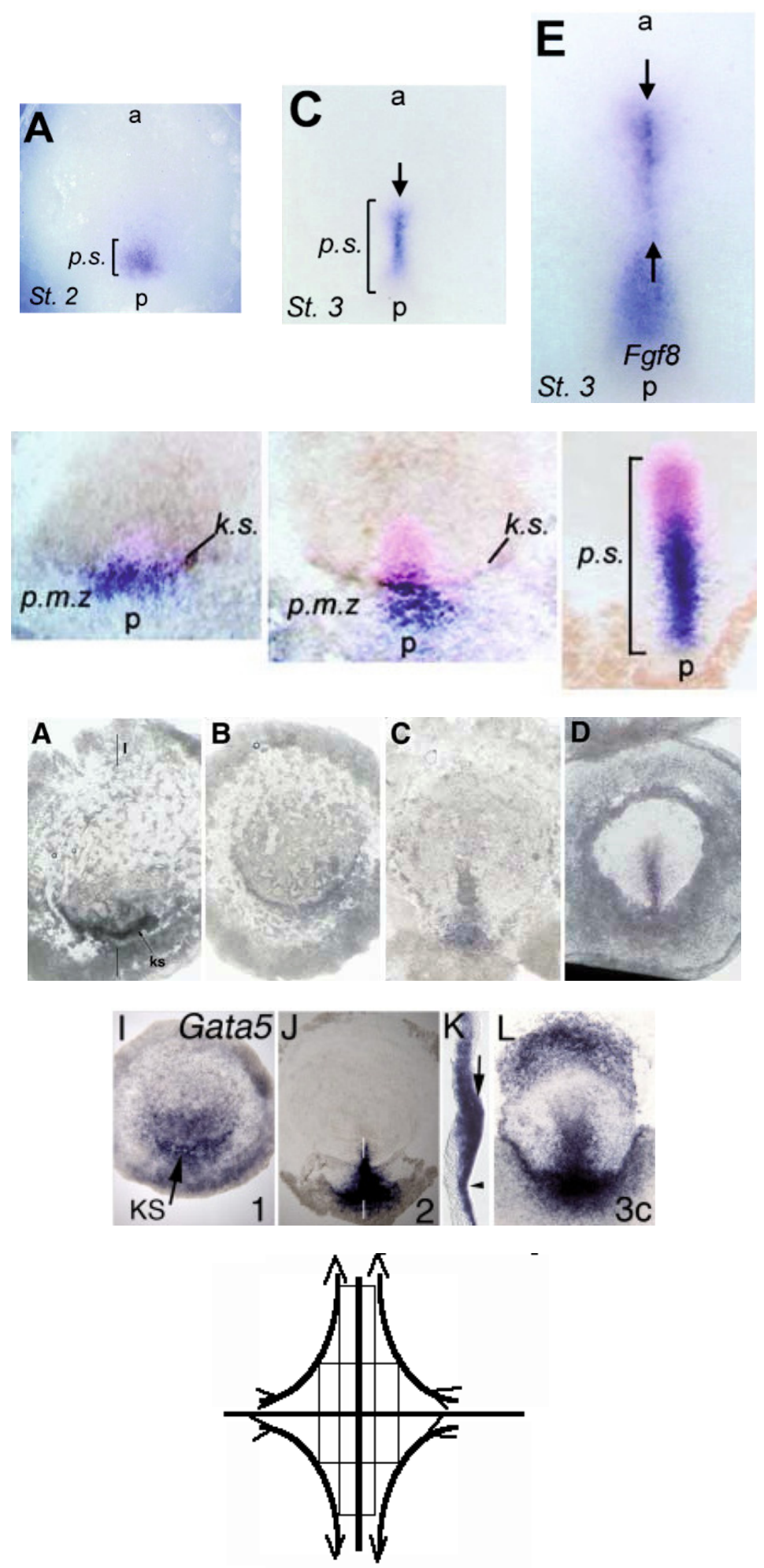

Fig. 31. (Color online) These images show several stages, at shortly different times, of concentrations of genes, as stained on different embryos, forming a stroboscopy of the developmental process. During primitive streak (p.s.) formation, the pattern of expression of Fgf8 (top) transforms from a circular spot around the contraction area, into a large "8-shape" centred on the presumptive navel area, between stage 2 and 3 (Hamilton \& Hamburger stages of embryo development) from reference [163]. A similar advection pattern is obtained for other genes such as Wnt8c and chordin (middle) or Clef1 or Gata5, which transform from a horizontal "bar" or "oval" to a vertical "bar" [162,165]; see also many early stainings of the database GEISHA. All genes actually seem to follow an advection into a hyperbolic flow (bottom, in a lagrangian view, the hyperbolic flow is elongational, and transforms affinely a spot into an elongated bar positioned along the median axis). 

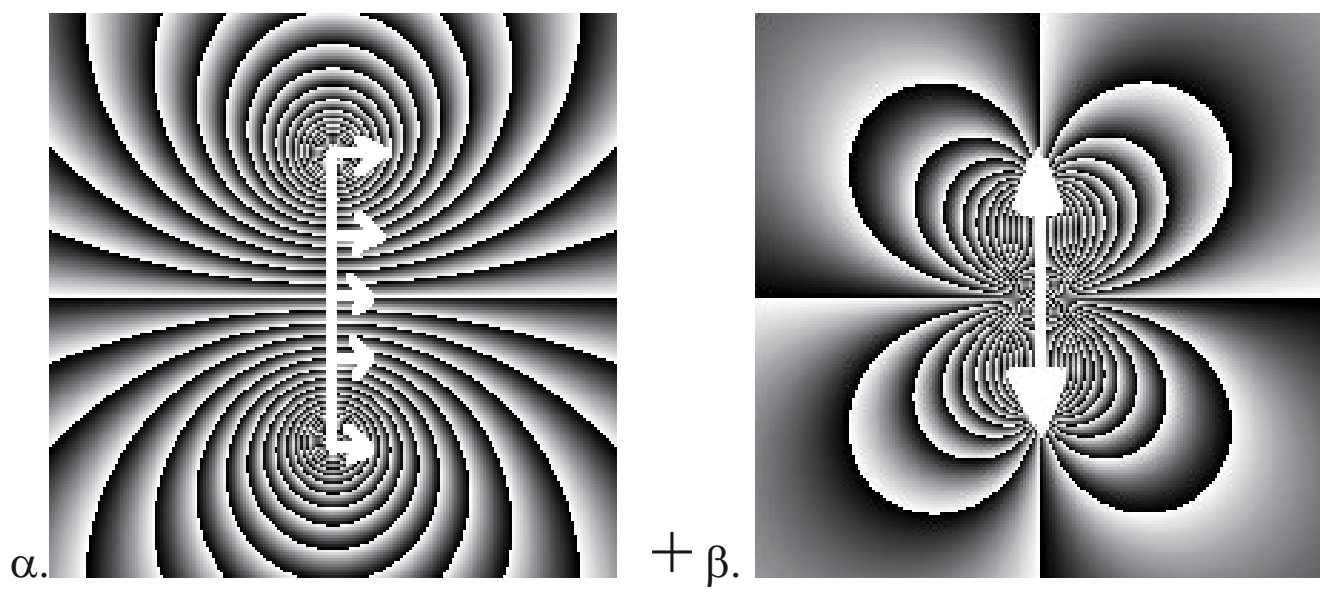

Fig. 32. A simplified picture of the rate-of-deformation field in the embryo is given by the sum of a contraction, and an expansion along the body axis, which describes a convergent-extension with identical cells (Eq. (12)). (The lines represent iso- $\Psi$ values of the stream function, the graded areas the gradient between values, in dimensionless form. Such parameters as force magnitude, viscosity, and thickness of the blastula will scale the problem).

stress, which is strictly neutral at the moment of perfect closure of the primitive streak, becomes compressive perpendicularly to the AP axis. Hence the tissue buckles and folds. Therefore, it is completely natural that the primitive streak recession (descent of the zipper, neutral stress) is followed immediately in space and in time by formation of folds (the neural crest, positive stress), giving an erroneous impression that the tip of the streak (Hensen's Node) is "inducing" the embryo body as it descends.

In addition, the entire embryo tissue is transported in the intercalation flow and produces naturally the large "8" around the embryo folds (Fig. 33). Moreover, the existence of a limited extension in the anterior part of the initial primitive streak, implies a finite length for the intercalation parameter $f$, while the primitive streak extends all the way down inside the caudal area. If we advect a blastula-like loop of tissue in the "extension" ( $\alpha=0$, $\beta=1$ ), we immediately see that the loop flattens in the direction of the navel, while the re-circulation of the flow lines induces a progressive closure of the "neural crest" in the middle of the body around the navel, with a descent of the closure of the neural crest towards the tail bud, while a furrow remains wide open in the "head" region. This may explain why a head exists eventually on the anterior side, while the neural crest closes completely at the tail side, a fact which has no explanation otherwise. Also, since the tail part is closer to the caudal edge of the blastula, the winding of the epiblast towards the body is more rapid in the caudal area, hence explaining why hindlimbs will form before forelimbs. Molecular stainings will be advected in this flow, as quite clear in Figure 9, or in database GEISHA.

All this is a consequence of hydrodynamic conservation laws in a viscous sheet. In addition, the neural folds move forward and push sideways the ocular regions, in a hammer or horseshoe shape formed by some sort of a "trail" of the fold deformed in the given flow, as classically known in developmental biology (See data base GEISHA especially
Hamilton \& Hamburger stages 5). It is known from graft experiments that the entire area of the flow trail in the head region can potentially give retina tissue [167]. The formation of the eye is a matter of progressive physical, lateral evagination of optic vesicles. Due to the horseshoe shape of the trail, eye precursors drift and get positioned in the actual eye cup [168]. However, zebrafish mutants which have a much reduced trail, and a head which protrudes more ahead than sideways are cyclopic, they have a single eye ahead [169], this is ascribed to the fact that the eye spacing is spatio-temporally decreased by modifications of genes (kny and Tri) which affect the hyperbolic flow. When the size of the "forehead" is too small, a bifurcation from a "peanut shape" eye to a single centred eye occurs such that a single eye is formed on the forehead. This situation is quite reminiscent of the limb development: as for limbs, an entire field is "competent" for development of some parts (eyes, limbs) but these will only grow out at a specific localized areas, which are concomitant with curved bumps (see Sect. 7.4) defined by geometrical and physical constraints.

This suggests that the bilateral symmetry of the eyes, and of the face is a hydrodynamic consequence of the flow magnitude which generates a wide horseshoe form of the presumptive head region. In more evolved vertebrates the horseshoe head so to speak "inflates" above the spine to give craniates. More primitive animals like Tiktaalik have a flat horseshoe head shape.

To end this section, let us note that the global hyperbolic flow flattens the tissue along the AP axis in the region of the presumptive limbs (quite visible in Fig. 22, in the region of the hindlimb), while the tail apex moves more caudally along the AP axis, and progressively closes, creating 4 windings away from the presumptive navel, 2 above and 2 below. Therefore, the formation of the body plan may be a direct, and quite simple, consequence of the flow pattern, with several biomechanical parameters of genetic origin, and many events described in biology by 

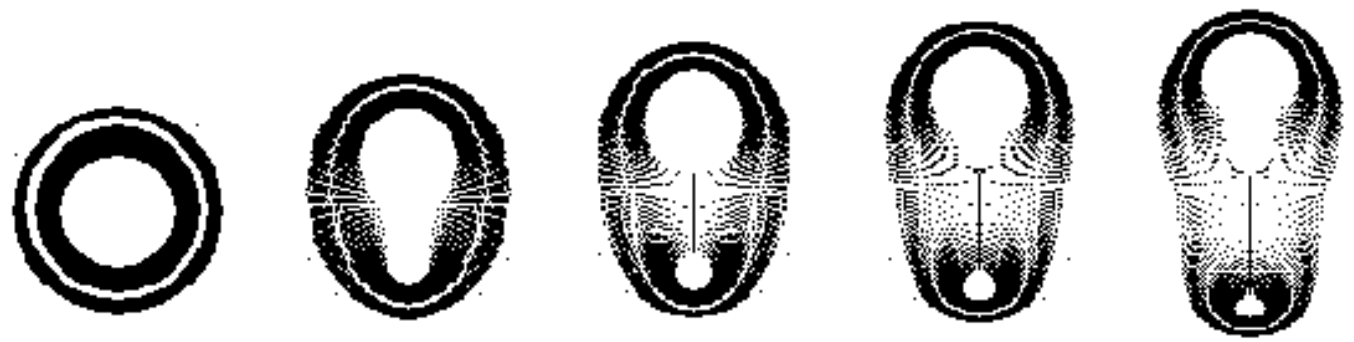

Fig. 33. Transport of a circular pattern in a generic "extension flow", with a navel positioned arbitrarily towards the south of the initial blastula. One sees the progressive collision of the circle along the presumptive neural crest. The first region of the embryo to close is the central area (around the navel) Because of the caudal position of the Koller-Rauber's sickle, the tail fold (tail neuropore) closes completely as it extends caudally, while the head fold remains wide open. This is apparent here in that the keyhole or "8 shape" of the second snapshot, is elongated so much in the AP directions that the bottom circle of the eight vanishes progressively, but not the top circle.

different morphogenetical stages may actually be just the consequence of the transport in the flow. What the calculation shows, is that apparently very complex motions, can boil down to very natural hydrodynamic flows. The hardly intuitive character of hyperbolic flows may let one think that embryogenesis is very complex. Integrating in time the trajectories in a non trivial, but simple hyperbolic flow, suggests that, although the true equations may be slightly more complex technically, the phenomenon itself is deceptively simple.

\subsection{Discussion of he biomechanical origin of limbs}

As explained above, the positioning of the limb is still an open problem, from the developmental biology point of view. We understand that the origin of lateral plates (the "limb field") is crucial, since these will define the positioning of the normal limbs, and even many details such as orientation of the limbs, if not their kind (forelimb or hindlimb). The "commitment" of the limb may well be related to the exact cellular distribution in the cellular field existing around and inside the lateral plate, prior to the outgrowth of the limb bud. Tetrapods are defined by the existence of four lateral plates, which eventually develop into different limbs, these plates find themselves advected naturally into their own "field" which participates in anisotropies, inductions, cellular traffic, etc. of the limb paddle. Understanding the overall orientation should be possible by addressing the deformation field during morphogenesis. It must be observed that the orientation of the tissue (the mean direction of cells and fiber bundles) at very early stages is an important component of morphogenesis [126,170-172]. Stopak et al. [170] show the crucial fact that "... within developing tissues, patterns of forces exist that are capable of physically rearranging collagen and determining its long-range order". Rotation of piece of tissue disrupt completely the growth, therefore "position information" [171] is actually not a scalar quantity, but more a vector or a tensor. When limbs are produced ectopically with a localized spot of growth factors, it is generally aberrant, although it "looks like" a limb (one ex-

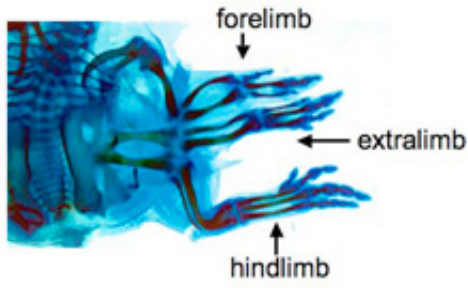

Fig. 34. (Color online) Ectopic limb, created in between forelimb and hindlimb by a soaked bead, shows a straight pattern (no flexion at the "knee-elbow" joint.). From reference [77].

ample of ectopic limb growth along the flanks of a chicken in Fig. 34).

Let us return to the establishment of the limb position. A typical Carnegie stage 14 human embryo shows clearly its limb buds, 2 forelimbs, 2 hindlimbs (Fig. 35A). If one winds backwards the development of the limb, e.g., hindlimb, one finds that it originates in a roundish tissue thickening located just below the presumptive navel area, for the hindlimb, and above for the forelimb (Figs. 35B, 35C, 35D its a chick). If one winds again towards the second day of development (of a chick embryo) one finds that the lateral plate originates in the rotational engulfment of the epiblast ${ }^{45}$ towards the median anteroposterior axis, while the axis itself extends caudally during the convergent-extension ${ }^{46}$ (Figs. 35D, 35E, 35F). Therefore, the rationale for lateral plate formation is that of a winding of the epiblast towards the body axis, while the body axis extends caudally (Fig. 36).

A typical image indicating how lateral plates may be "deposited" is found in [66]. Figure 37 shows the limb buds along the AP axis of a direct frog embryo (a frog which develops its legs in the egg, not at a tadpole stage). It shows that the lateral plates find themselves in the continuity of a line winding caudally, and rostrally, around a neutral point located half way between forelimbs and

\footnotetext{
45 In the early blastula, at a stage when the embryo is a flat disc, the top foil is called epiblast, the bottom foil is called endoblast.

46 This is the name of the hyperbolic flow in biology.
} 

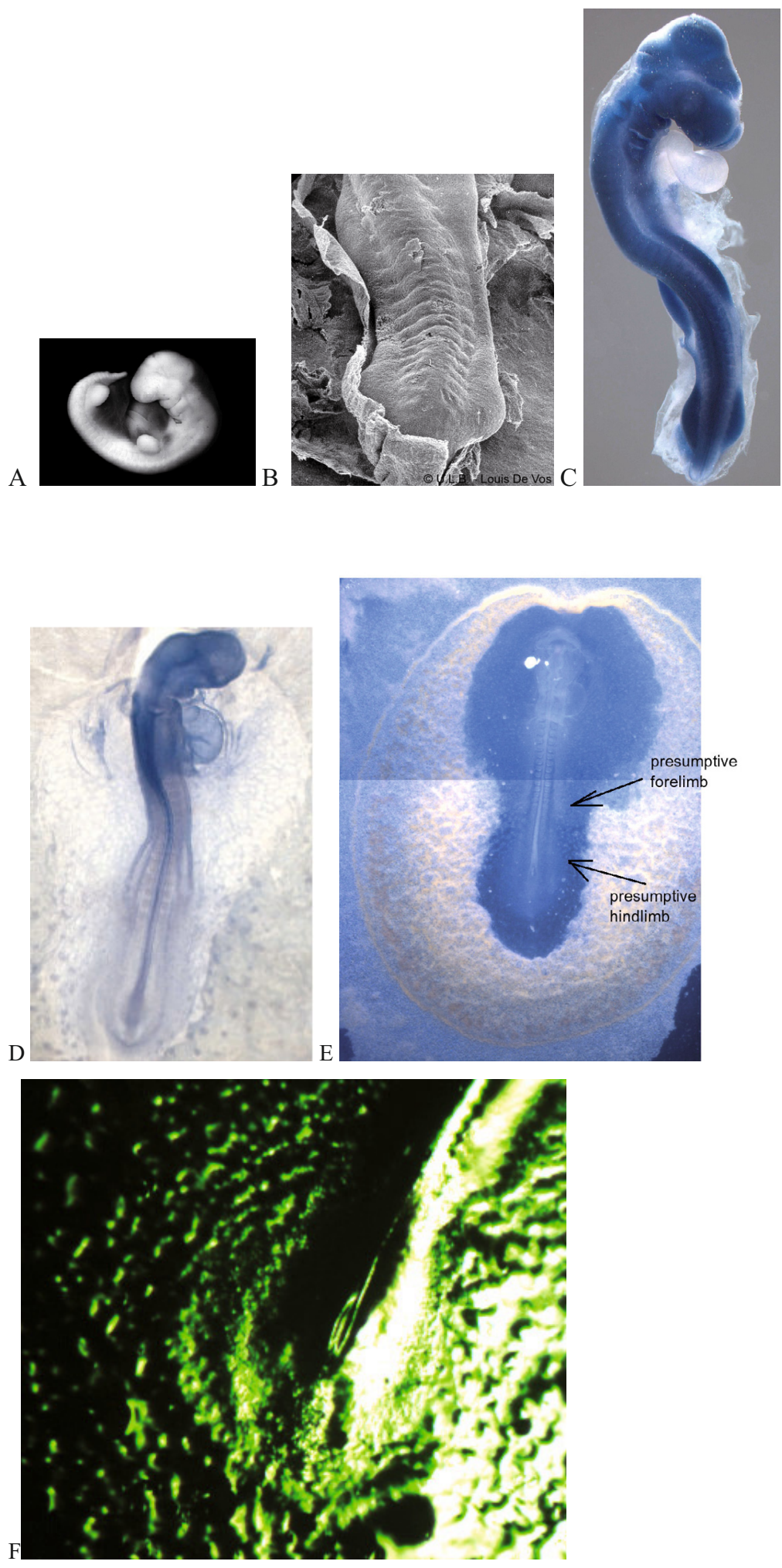

Fig. 35. (Color online) (A) typical Carnegie stage 14 embryo (human, Dr Mark Hill website, UNSW medical school) showing the limb buds. (B) Typical chicken embryo (stage HH19, ULB Louis de Voos web site) showing the bumps on the flanks (early lateral plates). (C) typical chicken embryo, showing the lateral thickenings forming the lateral plates (Geisha database). In (D) stage $15 \mathrm{HH}$, the thickenings are only faintly visible. In (E) (courtesy Alia Al-Kilani), they almost vanish, but they appear as a winding towards the posterior, or anterior part, the presumptive navel being in the middle. In $(\mathrm{F})$ the winding in the presumptive caudal plates appears more clearly. In this image shadowgraph surface imaging was used to show that there is an uplift of the epiblast in the presumptive hip-region, which accompanies the rotational engulfment of the epiblast towards the median axis. 


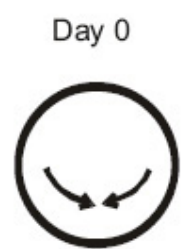

Blastula
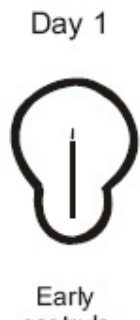
gastrula
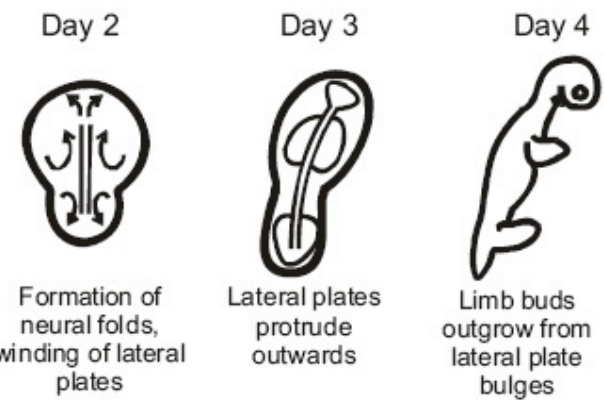

Fig. 36. (Color online) Left, description of the process of tetrapod development (chicken). Right the flow in the early blastula (AP axis is horizontal). The lateral plate originates in the winding of the epiblast tissue converging towards the body axis, while the body axis extends in the antero-posterio direction. The body axis extends caudally, in the tail region, and anteriorily in the head region, thus creating a strong winding of the lateral plate mesoderm, and related ectoderm folds away from a neutral point which is the presumptive navel (from Ref. [173]). Many molecular stainings in the caudal area just stain the actual geometry of the engulfments (see for example Tbx4 in Fig. 9 above).
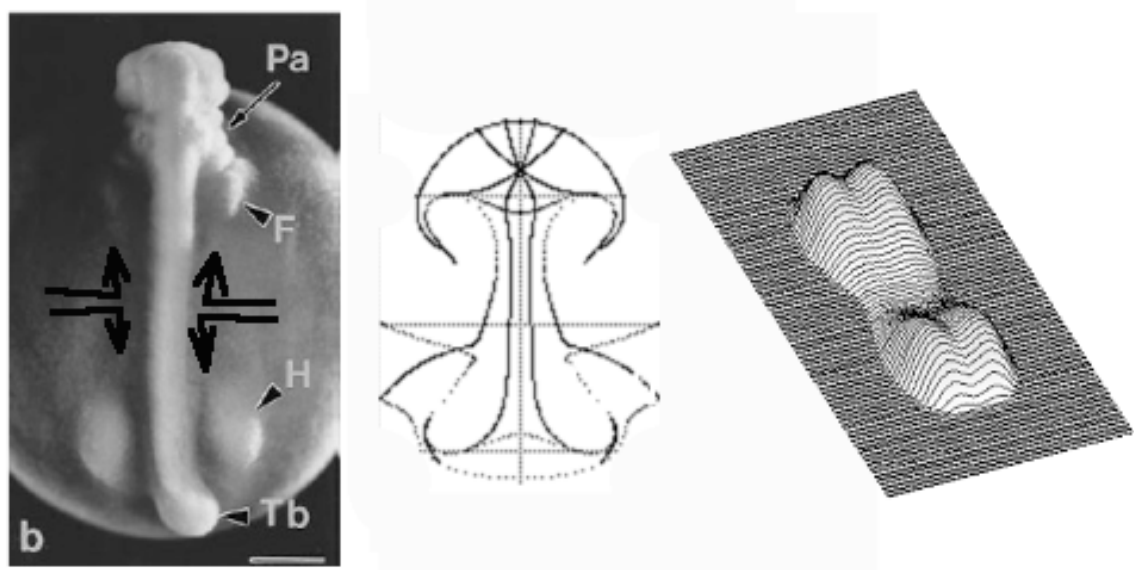

Fig. 37. Left, from reference [66], limb positioning in a direct frog. Middle and right, explanation of the anlagen ${ }^{48}$ of limb buds, as a consequence of the vortex motion in the blastula. The epiblast lifts up as a consequence of von Karman buckling under the hyperbolic stress [179].

hindlimbs. This situation is the one generated by the epiblast winding during gastrulation [16], in this view, the origin of the lateral plates is entirely within the motion of the cellular flow which self-organizes in the shape of two vortex dipoles colliding along the median axis, as a continuation of the hyperbolic flow (Fig. 37 right) and lifting up the tissue in the areas of strong winding towards the median axis, by von Karman buckling. Meantime, the winding pushes the head and tail so much forward that they dive in a curled manner, thus inducing the ectoderm to fold in a pocket shape that generates the chorio-amniotic fold. The collision of such vortex dipoles is quite apparent on films of the early moving gastrula. Such a movie is visible on Keller's website (shown also at http://www. gastrulation . org movie 15-3), and on youtube (keyword gastrulation).

Therefore the $2+2=4$ distribution of limbs in tetrapods seems to be a direct consequence of the typi- cal hyperbolic nature of the flow around the navel region, and so is the transition from anamniotes to amniotes. In addition, the overall orientation of the cellular fields in the area of the limbs may be a consequence of the flow winding.

If we assume that the limb bud will emerge from the central part of the winding, as it collides along the flanks, we see that the more proximal part will form out of the more bent parts of the lateral plate. As a consequence, the differential outgrowth of the lateral plate will progressively "blow up" or "reveal" the structure of the initial winding. This is why the limb bud of the hindlimb will be formed of elements impinging on the pelvis upwards, and then descending towards the paddle, while the limb bud of the forelimb will be formed proximally by a first segment descending from the scapula, and this descending element will next rotate upwards towards the forelimb paddle. Pelvis and scapula will retain the general outlook 
of the motion. A process of internal segmentation which we shall not discuss here, creates the bony structures, but on an overall limb form which is not itself segmented.

It is therefore clear that different parts of the embryo arrive where they have to by the flow, and that the flow has to be calculated by the laws of physics. The windings and folds of the early embryo have a direct impact on further growth, by fixing the boundary conditions and the initial cell orientations in all organ rudiments prepared for further growth, hence providing this coherent textural pattern associated to muscle, tendons, ligaments orientation, etc. which has been remarkably imaged by Kardon [172]. Therefore, there are no physical grounds in saying that a single restricted area such as Hensen's node is the "organizer" of the entire process. For example: the closure of the neural crest at the tail, the opening of the neural crest in the cephalic region, and the position of the limb buds are linked to the way the flow field recirculates, Also it is quite clear why ectopic limbs are generally not normal, since they are not positioned by exactly the same gentle convective pattern.

More specifically, it has been shown that the lateral plates protrude out during rotation and convergence of the epiblast towards the median axis. Mechanical measurements on the lateral plates show that they correspond to low stress areas, as expected for bent areas [173,174], such that cells in the limb field actually have a special "physical" phenotype. Therefore, in addition to possible chemical gradients which are stretched and reshuffled by the flow (advection-diffusion), such as Chordin, Hoxc6, $F g f 8$, Gata, etc. the physical force that winds the tissue in the areas of the lateral plates lifts them up by mechanical force, preparing a possible outgrowth, if growth factors are produced continuously. If they are not, limb growth can be interrupted at any time, giving the tremendous list of experimental assays, which show any type of limb, from a simple skin fold remaining along the body, to well formed limbs. However, if growth of the animal does continue, after the collision and winding, the first area to grow will be the limb buds of the lateral plates. These may inherit from early mode lockings the aspect of a digital arch, with regular rays.

\section{About segmentation}

While the embryo body forms, it also segments in what will progressively become cartilage condensations and bony elements. Indeed, it is often stated that a subtle interplay of chemical waves generates from the base of the neck down towards the tail the body segments of the vertebrates (early precursors of vertebrae, ribs, etc.). A considerable work is dedicated to the study and analysis of the genetic determinants of this genetic clock [175]. There exists a possible erroneous concept conveyed by the genetic clocks which are believed to generate these body segments in vertebrates. In effect, it is true that this segmentation wave descends from the presumptive neck down along the spine. However, there is ample Scanning Electron Microscopy evidence that, in fact, the body is already
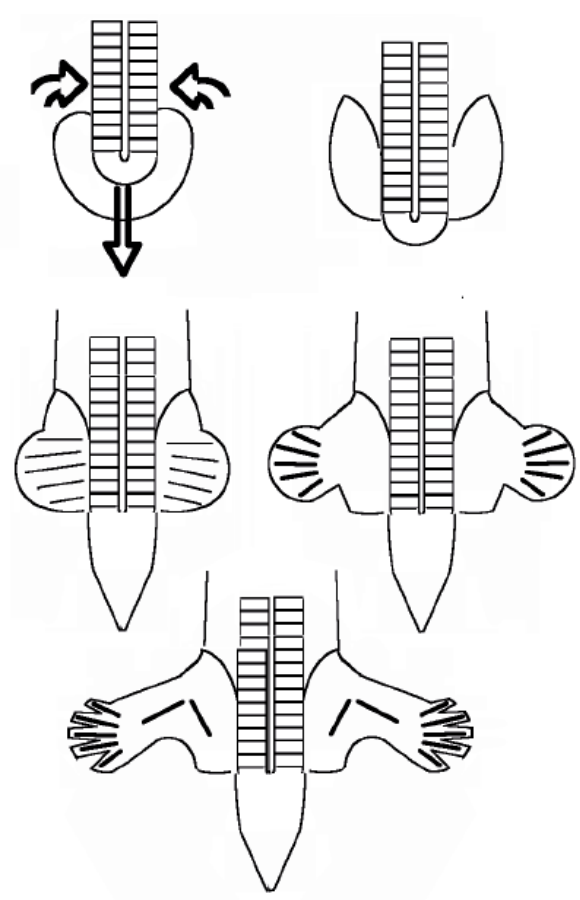

Fig. 38. Explanation of the global orientation of the limbs, in addition to their positioning. The hyperbolic flow winds the lateral mesoderm, and flattens the folds along the flanks. Progressive outgrowth of the most protruding part of the lateral plate will progressively reveal the winding pattern, and form limbs having the anatomically observed orientation (elbows flex rostrally, knees flex caudally). The number of digits will be locked onto the somite (vertebrae) number, at start of outgrowth. Typical pelvis shape is the result of the global flow.

segmented into somitomeres ${ }^{49}$, which are precursors of the segments, at the stage when the segmentation wave proceeds $[76,176]$. This segmentation wave seems only to amplify a pre-existing segmentation of the body, in somitomeres. The somitomeres are lenticular, stellar, foci of mesodermal cells which appear at regular intervals from head to tail, as the primitive streak closes [176]. They are positioned along the folds of the embryo body. Experts in the field ascribe this regular segmentation of the fold to the shear occurring in the area of the tip of the primitive streak $[177,178]$, and personal communication with Prof. Jacobson]. It was once thought that Hensen's Node was organizing the somitomeres pattern as it recedes. However, it was shown that HN is not actually necessary for formation of somitomeres [177]. In addition, when the embryo is split, the somitomeres form everywhere at the same time, and not in a sequential fashion following HN [178], thus suggesting a direct effect of shear along the fold (some sort of buckling). Such a segmentation process may be described by buckling equations of the von Karman type [179], acting on a sandwich of ectoderm and mesoderm. Such bilayers are intrinsically unstable, and buckle above a threshold of instability. Such models

\footnotetext{
49 Precursors of somites, themselves precursors of vertebrae (one vertebrae forms out of 2 somite halves).
} 
have been introduced recently for epidermal ridges formation [180].

\section{Consequences for evolution of the craniates}

In this section, I will simplify the hydrodynamic problem, and show how basic physics constrains the developmental pattern of the early embryo. This developmental description leads to several important evolutionary consequences, since all animal species emanate from individual animal, hence from possible embryonic modifications.

First, the description by a saddle-point given by references [121] and [131] can be approximated by a hyperbolic flow around the presumptive navel. While the limbs are associated to the areas of stronger winding, in the area of the saddle-point itself, the stream function has a hyperbolic shape hence a linearized form k.xy [173]. This shows that the displacement rate has opposite values around the saddle-point. In this situation, although two-dimensional, the flow is controlled by one degree of freedom only. This is to say that the topology of the problem, coupled to the conservation law, forces the flow pattern as much as if it were inside a tube. The family of hyperbolic flows collapses onto a master flow map, with a prefactor $k$. This means that during a given developmental process, either a tetrapod is formed, in which case the entire genetic activity is projected onto a given value of $k$, or something else is formed. As the flow pattern is transformed into an animal by the folding process, we see that the final shape of the animal will be related to the magnitude of $k$, which is proportional to speed of cellular flows.

Simple mathematics shows that around the saddlepoint, the deformation is purely elongational (in a lagrangian view where we follow the fate of a piece of volume): because of the linear form of the components of the displacement rate, a square centred on the saddle point is deformed continuously into a more and more elongated rectangle (Fig. 39), although each point follows a hyperbolic line.

This gives a direct clue of why almost all molecular stainings at this stage correspond to progressive elongation of pools of chemical along the presumptive body. This also explains why the body of a given animal has an elongated shape centred on the navel, since making an animal consists in elongating or shortening the body away from the navel, while thinning down the animal in the leftright direction. The transition between lizards and snakes, or carp fish and anguids becomes a simple one-degree-offreedom problem, and all genetic parameters are mapped at first order onto the value of this parameter, which acts as a cursor along the degree of freedom. Of course, the mapping onto a given value may correspond to entirely different genes, thereby favoring "evolutionary convergence".

One experimental clue of this phenomenon is observed by the phenotype of the chato knock-out embryo [181]. When the gene chato is knocked-out, a less elongated, more "squarish" embryo is obtained. This does not mean

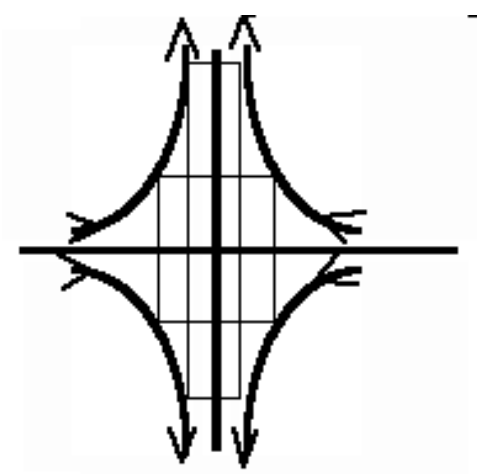

Fig. 39. A hyperbolic flow is elongational. Although all trajectories follow hyperbolas, the vector field components are linear in $x$ and $y$. Therefore, a given shape, say a square, is continuously deformed into an elongated rectangle, with increased aspect ratio. This explains simply why body formation is a matter of a circle becoming an elongated form. It gives a clue of how lizards become snakes, the bodies of the species "downstream" correspondig to a stroboscopy of a dimensionless dynamic flow, by modification of the prefactors.

that there exists a gene of "squareness" of animals, it suggests that the development is a physical process with its own dynamics anyway, with prefactors such that when these are modified, shapes are obtained along the family of possible shapes within the dynamics. The description of the transition between lizards and snakes seem to fit such a concept (especially considering the elongation of the pelvis in snakes which have vestigial limbs [49]); the observation by the authors that the body of snakes "resembles a stretched thorax" of a lizard is natural within the physical explanation. This process of hyperbolic flow may also contribute to explaining the shift of Hox gene domains between species, such as the 7 vertebrae shift of the thoracic segments of chicken, as compared to mice [11].

In addition, the hyperbolic form of the stream function is associated to a pressure map in the shape of a saddle. Pressure is high along, and far away of the left-right axis, and descends away from the saddle point along the body axis, which may be a clue of why genetic expressions (Fgf8, Hoxc6, Nodal, Chordin, Goosecoid, Shh, etc.) have the same geometrical profile (and not an antero-posterior gradient).

Now, as we observe that the flow around the saddlepoint gives a one parameter family of streamlines, we can continue the rationale. Actually, this hyperbolic flow is formed by the collision of vortices. Simple flow dynamics tells that flow singularities tend to collide and move away like particles $[16,182]$. Indeed, when vortices are singular solutions of a hydrodynamic equation, with a core positioned at a given point $M_{\mathrm{i}}$, introducing several such solutions into the hydrodynamic equation and expanding the dynamics in terms of the positions $M_{\mathrm{i}}$ shows that at first order, the transient flow dynamics amounts to a flow of each singular vortex, defined by the velocity $d M_{\mathrm{i}} / d t$ in the flow created by all the others. This classical and spectacular property is an "emergent" property of field 


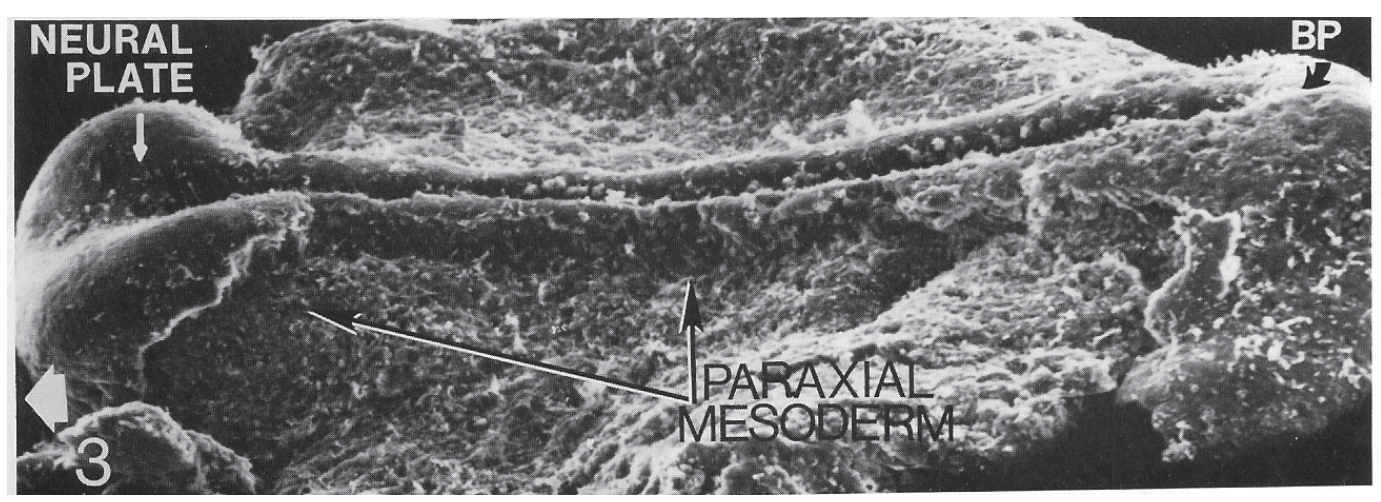

Fig. 40. Image of the early body fold showing to the left the open folds of the presumptive head. To the right, the tail fold is already closed. From Meier and Packard, reference [96].

equations. In presence of singular solutions, the flow of each singularity is found by advecting the singularity in the flow created by the others. In the present case, on each side of the saddle point, vortex dynamics tend to move the cores of the vortices away, as they collide towards the median body axis. This explains simply why the body tends to stretch and thin down along the head-tail axis, while the limbs get closer to the body; by the same token pelvis and shoulders tend to rotate. Also, the growth is not from head to tail, but tail-bound and head-bound, away from the navel.

This early flow orients further embryonic growth. It is a simple fact that the tail may be viewed as a one dimensional entity. It is less simple to observe that, in the direction of the limbs, and of the head, a similar reduction of the problem occurs. If indeed the flow dynamics selects the points of outgrowth, at the moment of outgrowth, the limb is a $3 \mathrm{~d}$ entity in interaction with the flanks. At that moment complex mode-lockings may occur, such as pentadactily. However, as the limb extends, cellular flows become well organized with a liquid-crystal like behaviour [172], i.e., there is an established cellular orientation $\mathbf{n}$ with cell-to-cell correlations. This correlation is likely associated to a physical interaction between biopolymers (hence energy terms which give a penalty to $\operatorname{div}(\mathbf{n})$ terms). In this instance, the limb extension will be constrained by the oriented phase of the tissue, and this is amplified by the fact that cells tend to follow, by durotaxis, the underlying collagen bundles of the extra-cellular matrix [126].

It is a classical observation in paleontology that tetrapods are first plantigrades, then digitigrades, then unguligrades. It seems that, regardless of chronology, in the space of forms, these anatomical drifts correspond in first approximation to a progressive extension of a fixed "scaffold", as stated by Darwin, accompanied by a reduction of digits which may remain as vestigial digits on the sides of the hooves of unguligrades.

The one dimensional character of the cell flows during early morphogenetic movements is passed to the cartilage pattern, and further to the bones [184]. Deep cartilage orientation is perpendicular to the subchondral bone direction, and becomes parallel to joint surface, under mechan- ical forces of reorientation [185]. The orientational nature of the cartilage is quite important for the proper extension of the bones $[172,187]$. Genes which affect the correct alignment of cartilage material induce very serious bone abnormalities, dwarfism, and severe developmental conditions [186]. The bat gene promoter mentioned in introduction (non coding zone of the Prx1 gene) which promotes longer forelimbs in mice is actually a promoter of cartilage growth. In this view, cartilage "plan" is controlled by the texture (the scaffold of collagen constraints cells, such that traction forces can change magnitude but not orientation), and modifying the magnitude of growth factors stretches the limb in the direction of the existing texture, which gives to Darwinian evolution a deterministic flavour (animals seem to follow "tendencies", and similar forms are re-iterativey obtained at different times, or on different continents), as observed by Darwin himself. In this spirit, making a proper animal is a matter of cartilage texture.

Cartilage being very well organized, it constraints further developments along the orientational arrow of the texture, itself inherited from gastrulation motion and segmentation. Such textural constraint is also known to position blood vessels along dorsal segments, muscle fibers, or skin $[131,148,171,188,189]^{50}$. This may be, indeed, a quite simple explanation of the "scaffold conservation": it would be a consequence of the constraint by the physical process. Any other explanation, such as assuming a wide variety of possible genetic inductions, and then supposing that most of them were put off by selection, requires to understand why all the other body plans were not evolutionary fit. Stated otherwise, it is actually not physically possible for a limb to evolve a vertebra-like or a skull-like bone at the wrist, or a set of ribs instead of the fingers, although all these bones are fundamentally made with the same genes.

It can be said that the hyperbolic flow prepares the young embryo in a state where cells of each part are deposited with a specific orientation, along specific areas for further development. Much like a starfish, the vertebrate

\footnotetext{
50 In this elegant work, Krenn and Wachtler rotated small square pieces of avian mesenchyme, they observed a very significant disruption of cell migration for $\mathrm{HH}$ stages later than 24.
} 

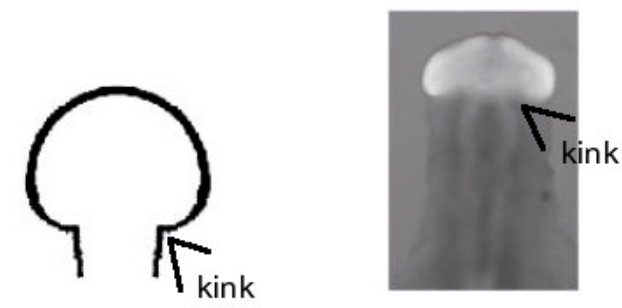

Fig. 41. The neural plate region will face a recirculation of the fold (a "trail"), such that there will be a kink in the fold contour. The image to the left is a simple calculation of kink formation in a circular form advected in a typical convergent extension pattern (already visible above in Fig. 33). The formation of this kink in the presumptive head area is a physical consequence of the deformation field.

is also a star, but a six-armed star, one arm being the tail, 4 other arms being the limbs, and the last arm being the head. If we now turn to the head, we see that the situation in this area is more complex, in that instead of closing up, the folds induced by the hyperbolic flow remain open.

However, as these folds grow and blow up, the problem becomes also a one parameter problem, among a family of dimensionless vector fields. Indeed, if a developmental process of any embryo has reached the situation in which the folds of the neural crest are formed, then cells find themselves along the folds of the neural crest in the cephalic region oriented perpendicular to the folds along the neural plate fold edges, as classically observed ([176,178,188] and references therein). Due to the dynamics of the flow field, the neural plate finds itself spontaneously closed at the notochord, in the region of the presumptive neck (Fig. 40, to the left), at the upper point where the somite region of the back meets the cephalic open folds, and it finds itself progressively facing an angular reentrance in the region of the presumptive eyes (Fig. 41).

In this situation, cells find themselves along the neural crest folds oriented upwards in a situation of upspring of a viscous flow analogous to the one given in equation (5) (Fig. 29B), but this time along the AP median axis, oriented vertically out-of-plane. More specifically, due to the existence of the singular point at one end of the notopore, and in the eye region (the kink shown in Fig. 41), the flow winds around these two singularities, forming a vortex ring which injects the cephalic tissue upwards (Fig. 42).

Therefore the flow of the neural plate amounts to a cell injection around two pivots which tend to generate a roundish boundary that winds forward (above the eye) and backwards (in the presumptive neck region). Suppose, now, that the offsprings of an individual undergo some molecular mutation changing the biomechanical properties of the cells. Either the cells lose their oriented field, in which case the pattern of growth is completely disrupted, or they do retain this orientational aspect along the neural plate, and they arrive along the edge of the cephalic crest in the situation of a vortex ring injected upwards, anyway. Therefore, all mutations that do not affect the orientational aspect of cells along the crest provide an ini-

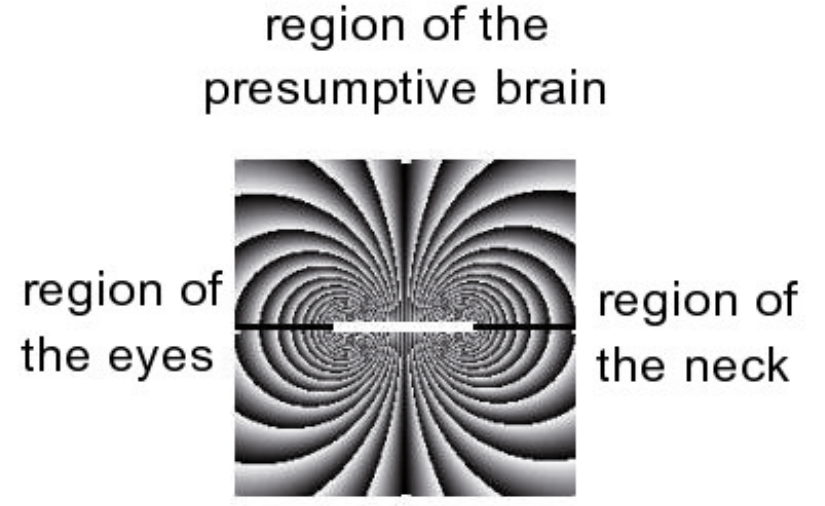

Fig. 42. In the region of the presumptive brain, the tissue is injected with a flow pattern which is analogous to a vortex ring, and it pivots around two singularities which are the base of the neck, and the eye cups.

tial condition for the cephalic formation which is topologically the same in terms of vector field. However, inside this topological situation, many parameters can be modified, especially, the thickness of the epiblast, the viscosity of the tissue, the time scale of the process, etc. Still, all these parameters will only affect the prefactors of the vector field, not its topology, which explains why such events as head formation may occur at completely different speeds, but still form similar shapes.

More precisely, the skull starts in embryos by minute vesicles of approx. 1 millimeter, and end in the human skull, by a cranium of diameter $25 \mathrm{cms}$ (Fig. 43). On the brain vesicle, the orientation of the stress field appears clearly through the mean path taken by blood vessels (Fig. 43 left). The situation encountered in the head can be reproduced in the yolk-sac, (which is completely circular) by welding an obstacle that constricts one half of the yolk-sac. Then the yolk-sac starts to rotate around the edges of the obstacle (Fig. 43). Instead of growing round, the yolk-sac pivots around the edges of the paper. In a head, the obstacles are the closure of the notopore, in the dorsal area of the neck, and the eye orbits.

In the morphogenetic space, no other degree of freedom than a dilation of the radius is observed. Cranium never give way to hands or feet, or ribs. All tetrapods (craniates ${ }^{51}$ ) have a cranium, in between small and large, but it is always a cranium. The dilation of the brain vesicle is again a one parameter family of shapes, which scales a dimensionless dilation process.

In the end, the cephalic vesicle will wind anyway upwards and around the pivots, but more, or less, depending on the exact value of the parameters. Therefore, the possible cephalic forms finally observed will be related by the winding of the flow around the pivots, as discovered observationally by DambricourtMalassé $[190,191]$ by careful analysis of the rotation of the sphenoid bones in the human lineage (Fig. 44).

\footnotetext{
51 Tetrapods without a skull have a flat head in a shape of a horse-shoe. Craniates have a head with a round cavity passing over the eyes.
} 

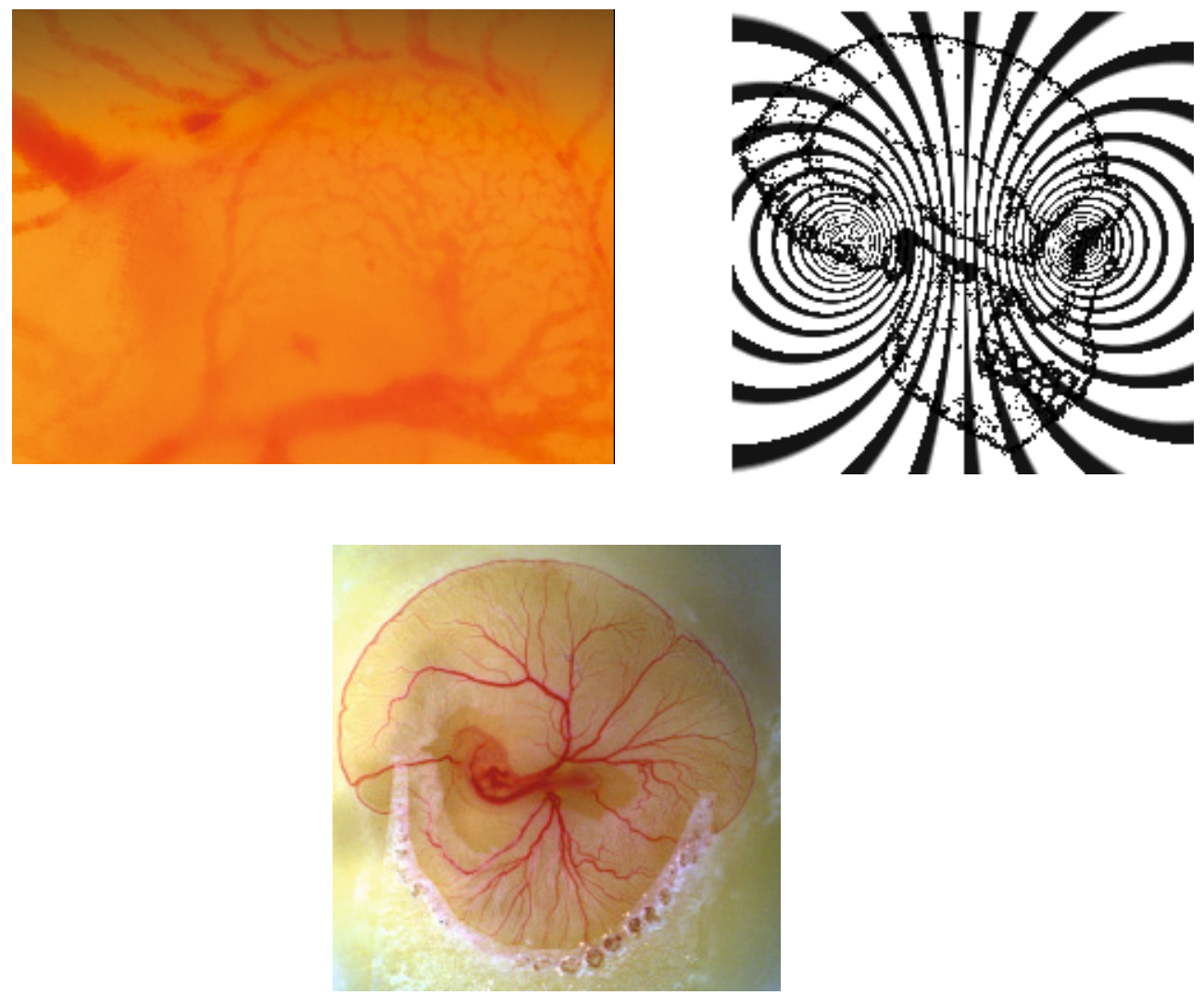

Fig. 43. (Color online) Comparison of a small brain vesicle in an early chicken embryo and a skull of and adult homo habilis. Left, a very young avian embryo, at approx. 5 days of development, close view of the brain vesicle (diameter $3 \mathrm{~mm}$ ). The pattern of capillaries in the early embryo head orients itself in the shear force [148]. Right, a drawing of an homo habilis skull, with a pattern of presumptive stream lines superimposed. The head material rotates around the ear orifice (roughly), and above the eye orbits. The pattern of vessels in the brain is similar to the pattern of vessels in the yolk-sac, except that it is 3D [148]. While the yolk-sac expands radially in a discoidal fashion (see Fig. 25), the brain vesicle expands in a spherical fashion. The situation in which the eyes and the ear orifice (closure of notopore) act as obstacles for skull expansion can be reproduced by placing and obstacle around one half of a yolk-sac. The yolk-sac starts to pivot around the obstacles (bottom).

In the head, indeed, it is observed that the floor of the head (sphenoid bone) flexes, with a rotation of the entire face, which winds and flattens clockwise with respect to the floor of the head, while the rest of the head (half of the brain, cerebellum) rotates anti clockwise and winds towards the neck. These motions correspond to a dipolar flow of the head tissue with an injection located at the floor of the head, and a vortex ring winding towards and above the eyes, and towards and above the ear duct. This motion generates a larger brain, enlarging both towards the dorsal part of the neck (atlas bone), and towards and above the eye orbits.

However, the winding of the tissue in the early neural plate implies a pulling action on the rest of the blastula, by conservation laws of physics, therefore, the winding upwards of the cephalic region has a pulling effect on the surrounding tissue which forms the neck, the mouth, etc. We see that, as observed by Dambricourt-Malassé, and confirmed by a simple analysis of deformation fields, the deformation of the blastula, will, in cascade, imply a global deformation of all parts of the final head which is obtained. If we turn to the case of a specific craniate, like the hominids, we see that, say, a homo habilis skull will evolve following a winding around two pivots which are for one located around the ear orifice, and the other in the region of the eye orbits (Fig. 45).

If we run the winding of the flow around the pivots either one way or the other, we find a deformation of the head, which is a consequence of the deeply rooted deformation of the blastula. We may speculate that there exists a dynamic phenomenon, inside the embryos, corresponding to a master flow map, which is revealed in the fossils as an apparent dynamical phenomenon through subsequent species shapes, because the genetic parameters stroboscope shapes by arresting the developmental phenomenon at different times. The flow dynamics transforms one form obtained in one species onto a form obtained in another species, as they should be related following the dimensionless morphogenetic field, and as announced in introduction (and so well exemplified by D'Arcy-Thomson, and pioneered, at a technical level, by Jacobson and Gordon [140]). In the case of hominids, this deformation leads either to an enhanced prognathism or a reduced prognathism, associated to a skull volume 


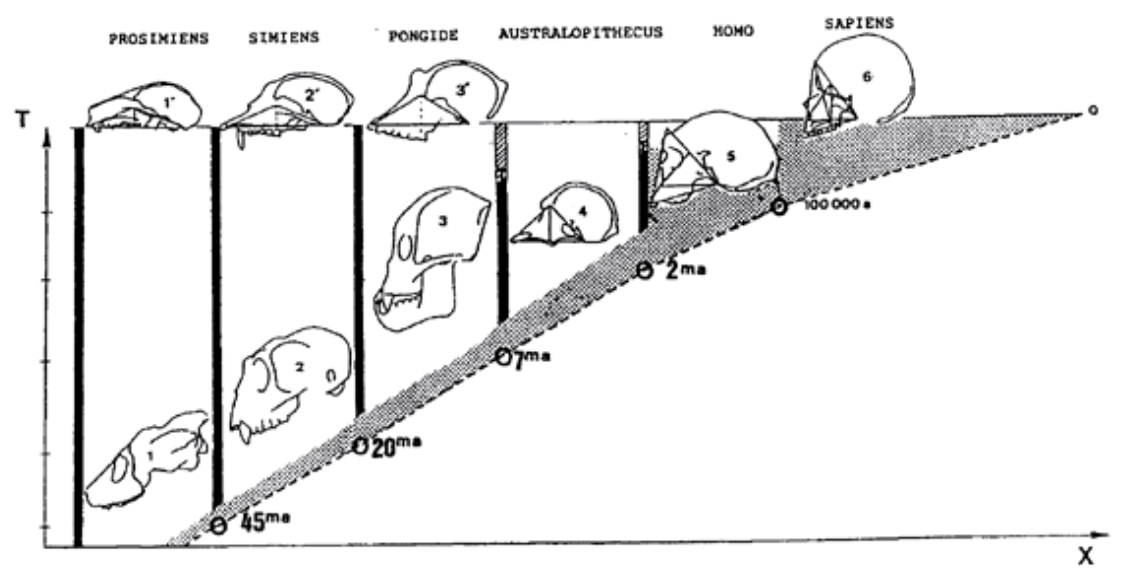

A

$\mathrm{B}$

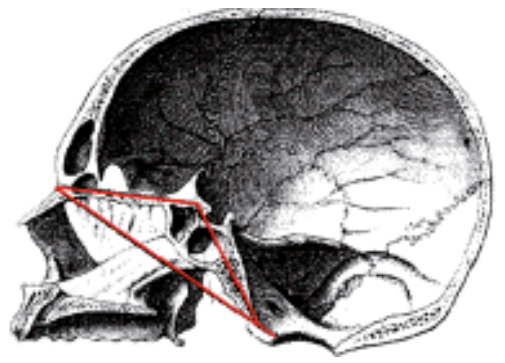

Fig. 44. The paleontological data reveal a progressive dipolar rotation of the head. The floor of the brain is engulfed upwards and the angle at the summit of a triangle passing by the atlas, in the neck region, and by the eyes (shown here in (B)) tends to become more acute. This is to say that the segment eye/brain-floor, and the segment brain-floor/atlas rotate counter-clockwise, and clockwise, with respect to the summit (with the head orientation shown in (B)). As this occurs, the brain volume increases, while the face recedes (less pronounced prognathism). This is the sign of a dipolar flow. As this dynamic pattern is observed during evolution, it seems to imply the existence of a deterministic, dynamic, process of humanisation in the space of shapes (Dambricourt-Malassé [190]).

increase or decrease. The jaw narrows or widens as it is pulled up or pushed down. This, again, fits the experimental observations and model of Dambricourt-Malassé, which she termed "inside story", as opposed to "east-side story", the scenario of emergence of humans in Africa, east of the Rift Valley. The phrase "inside story" has in fact a strict scientific meaning: there exists a dimensionless vector field, for the development of the skull, which is truly "inside" the developmental process, that is, in an abstract mathematical space.

Now, we see that in terms of selective advantage, the conservation laws of physics relate an increase in brain size with a decrease in jaw size. Therefore, if it is favourable to trade a mandibular advantage for a cognitive advantage, the forward motion of the vortex winding will be selected, as observed.

\section{Conclusion}

Reviewing the paleontological, genetic, developmental and physical data, shows that a dynamic picture of embryo development, resting on fundamental laws of physics, can be proposed. In this view, development consists in a continuum deformation of an initial formless animal which progressively changes shape by scaling up an initial symmetry breaking. Starting from such a symmetry breaking, a uniform behaviour of cells (constitutive equation) suffices to induce a deterministic asymptotic form, by low Reynolds flow. The dimensionless flow forms a general law, whose parameters have a genetic origin. Modifying the parameters shifts the animal forms along morphological diagrams which follow the streamlines of the flow. Although several aspects (phaners, metabolism, cognition, etc.) add to the problem lots of features which we have not addressed, there may exist a simple biomechanical rationale that explains in detail the global pattern of embryo structure in vertebrates. The formation of a streak, the invagination of the yolk-sac, the back and forth motion of Hensen's node, the formation of a body which has globally the shape of "an 8", the lateral position of the limb fields, are all very complex and important developmental questions, which in fact may be reduced to simple hyperbolic viscous flows in the embryonic sheets. These flows require an initial symmetry breaking and possibly genetic pools of molecules which overlap mechanical fields. The rationale of animal formation is that of a cellular flow which runs away by producing its own factors of self-organization.

In terms of complex fluid dynamics, much remains to be understood in the coupling of biochemicals with the 


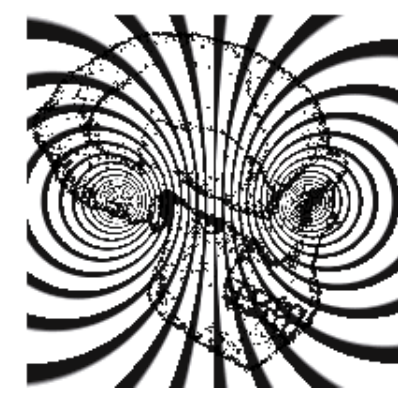

(a)
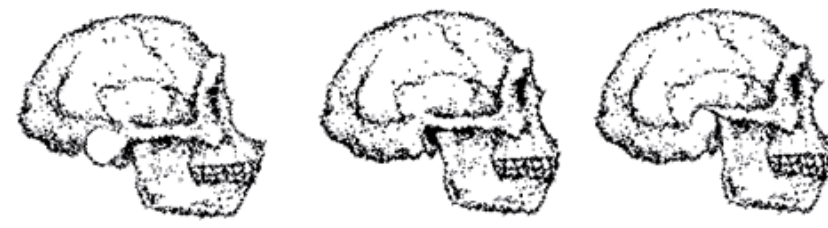

(b)

Fig. 45. (a) Super-imposition of the expected flow and of the corresponding pivots in the skull (one at the base of the neck, near the ear orifice, one in the ocular area). (b) Speculative evolution of the human skull following the model presented here, in a typical winding flow. Starting from a reference homo habilis (in the middle). (b, left) Retro-evolution, (b, right), evolution towards the future. One may obtain a more simian-like skull by winding the vortices "backwards", or a more human skull by winding the vortices "forward". More human skulls are characterized by a less pronounced prognathism, a brain which descends towards the neck, and a jaw that is stretched vertically.

true deformation field, but whatever the future progress, it appears that the formation of a vertebrate body may be topologically much simpler than expected. There exists a saddle-point in the early pattern of tissue movements. As confirmed by experimental an theoretical work, the overall pattern-formation of the embryo seems to be driven by a generic hyperbolic attractor, acting on a visco-elastic material. The formation of the vertebrate body comprises three steps: planar hyperbolic flow (low Reynolds), outof-plane buckling of a thin viscous sheet (quite reduced number of folds, about 2), propagation inside of a segmentation wave (with mode-lockings between parts). During these phenomena, the texture aligns in the main shear ${ }^{52}$, and orients tissue development. Although a difficult mathematical challenge, the phenomenon in itself is simple, and it seems to explain the strong tendency of bone "scaffolds" to simply stretch during evolution, except in areas of vorticity. The parameters of such a process are in a very small number.

\footnotetext{
52 Because embryos are formed of thin cellular sheets, the main shear is oriented in the direction of the cellular flow and acting on the in plane facets of an elementary volume.
}

If one considers the fate of the ingressing mesoderm, the flow pattern "induces" naturally the formation of a progressive circular yolk-sac, with cells that migrate around the anterior and posterior parts of the blastula. A circular animal is the simplest animal, for cells flowing away. It has been shown recently that Stokes flow can describe this circular organ [151]. It seems that an embryo is nothing but the same tissue, but in a state of convergence, instead of divergence. From a mathematical point of view, the yolk-sac is a hyperbolic inversion of the embryo, with respect to the navel. In alternative views, cells of the epiblast which epibolize, start immediately to migrate away from the antero-posterior axis by a chemical coincidence. In existing concepts, one needs a chemoattractant towards the body axis for the initial epiblast flow, which turns instantaneously into a chemorepellent, as soon as cells dive and make a U turn. While this may be the case, there remains to explain the origin of all the coincidences of the chemical expressions leading to cellular flows, invagination, U turn, etc. if not by the stress field (the morphogenetic field itself). In addition, not all cells migrate away, therefore there remains to explain why some cells reside in the notochord, while others migrate away, etc., which leads to apparent inconsistencies.

The presumptive body axis derives from an initially horizontal (Left Right) sickle, formed by cortical rotation, which is transformed into a straight vertical (Antero Posterior) line by a hyperbolic flow. As shown above, considering a uniform cellular behaviour along the epiboly line, and a uniform cellular behaviour along the intercalation line suffices to generate an entire body plan and a yolk-sac, very much similar to what is observed, at very early stages of embryo development. This suggests that it is in fact very simple to generate dynamically an "archetypic tetrapod", as coined by Darwin, and that cascades of genetic inductions with stringent chemotactic patterns might not be required. This is deeply rooted in the hydrodynamical field, which consists actually in writing the interaction between cells in a continuum medium. In Darwin' $\mathrm{s}$ book, it appears in chapter $\mathrm{V}$ that when there exists interactions between parts, the observed morphologies should be "independent of utility, and therefore of natural selection", but actually, the cellular interaction between cells is already an example of such physical correlations, governed by physical laws, and the global pattern is an emergent consequence of this interaction.

Very fine analysis of cellular motions have shown that if, indeed, a true difference can be made between advective flows and chemotactic navigation, these two components should anyway be of the same order of magnitude [158]. It is quite possible that one large share of the chemotactic component be induced by force gradients, as these are always present, and known to induce expressions of important chemotactic molecules.

A possibility arises, therefore, that embryogenesis be organized by a flow field of cells which exhibit a quite restricted set of behaviours. These behaviours, sometimes interpreted as completely different features, may be viewed as different outcomes of the same cause (physical 
stress acting as epigenetic cue). In addition, the pattern is constrained by the initial topology, such that inside an open set of parameters, genetic influences are shifts over a deep physical process, which is generic. The flow takes its parameters in the biochemical content of the embryonic tissue, which is related to all genetic expressions. But once the vector field is established, the parameters of the field appear as prefactors of a dimensionless problem, and the further evolution of the animal consists essentially of a flow in a field whose field lines confine cells along deterministic trajectories.

It is possible that the same rotational flow generates the lateral plates, the neural plates, the yolk-sac and navel, the amniotic sac, etc., by the same token, thus reshuffling a round blastula into a tetrapod, even surrounded by a bag. Especially, the topological description by a hyperbolic flow challenges the accepted view of genetic duplication of collinear clusters of homeobox genes. It may be possible that the 4-fold distribution of limbs in tetrapods be not a matter of chance, but a topological consequence of the symmetry breaking. That would naturally explain the observed chirality of limbs.

Ectopic expressions and the like, however spectacular they may be, often correspond to artificial situations which cannot spontaneously exist in a true embryo, since the localisation of FGFs, RA, Tbox or other molecules have to comply with the previous spatio-temporal organization of the cellular flow anyway, itself controlled by cell-cell interaction via the continuum material which the blastula is. The concept that morphogenesis is "constrained" by physics is inappropriate, since all deformation and flows, be it normal or aberrant, have to abide by the laws of physics (especially here, mechanical interaction and mass conservation). Of course the parameters of the process have a genetic origin.

Now, turning to evolution, we see that indeed this situation renders the evolution predictable, to some extent. Why is it so? For three reasons. First of all, once the cells are polarized, the type of flow is generic, and after arrival at the limb field, the tail, the neural plate or other parts, in a parallel position, they inject the tissue in an organized fashion anyway, or if they don't, the pattern is grossly aberrant [16]. Secondly, a given flow implies internal correlations between morphogeness of different parts, independent of the context. In the case of the head, the vorticity of the flow relates the expansion of one area to the crushing of another, therefore, independently of the biotope, there is already a correlation between the development of embryo parts, a fact well known of Charles Darwin (ibid. Chap. VIII, paragraph "Correlative variations", and also Chap. XIII paragraph "Morphology", Darwin ascribes correlations in forms between plant organs to pressure gradients, he even suggests that intrachorionic pressure may have an epigenetic effect on head size in humans), and sadly overlooked. In the case of the skull, the expansion of the cranium is related to the decrease of the jaw (and also to the transition from anamniotes to amniotes).
If the expansion of one part is favourable, even taking into account the crushing of the other, it is so in an open set of variables. Therefore, either the shape (e.g. skull size vs. jaw size) has reached an optimum, or, if it has not, the evolution will proceed following a motion which continues along the same vortex flow, since the parameters affect the prefactors, not the topology of the flow. In this set of parameters, the evolution is either finished, or predictable. What do I mean by that? If we consider, say, the problem of head evolution, or more simply, the problem of limb evolution, one may wish to plot on a chart the fitness of the successive animals as observed in the fossil record. For example limb length of equids, or skull size of primates, and find that, following Darwinian concepts, the fitness of the species increases, as a function of variation of the said trait. However, in this view, such a chart does not represent the time stroboscopy of any dynamical process, because the points along the chart correspond to the subset of selected animals, among all random developmental variations which gave animals, most of which were not selected. Finding a dynamics in such a chart is an a posteriori, retrospective, illusion. This is why, in classical Darwinian views, even if a series of points may be phenomenologically traced, they do not correspond to any predetermined developmental path; and they cannot be extrapolated in any way. However, the situation is entirely different if there is a master vector field of deformation, on which the genetic variants are all projected. In this case, the lengths of the limbs are the outcomes of a dynamical system in the embryo, and discontinuous variations of the genetic parameters map discontinuously the animal forms onto closely related animals with longer or shorter limbs (or smaller or longer jaws, etc.). While the genetic parameters may have discontinuities, the space of forms is itself continuous, and there exists a dynamic mapping among forms. However, if within a given environment, increasing the length of a limb was an evolutionary advantage, the process will deterministically continue, discontinuously (stochastically), as long as genetic variants are provided. But, in the specific case of the skull, a decrease in jaw size, is related by conservation laws to an increase of skull size, and vice-versa, by the vorticity of the stream-lines in the area of the neural crest. As a consequence, if the fitness of the animal is a monotonous positive function of jaw size (having a bigger jaw is an advantage), and a positive monotonous function of brain size (having a larger brain is an advantage), then the total fitness of "the head" is a matter of difference of an advantage minus an advantage, which are both correlated by the same morphogenetic dynamics. Needles to say, having no jaw, or no brain is detrimental. Therefore, if animals having larger brains are locally favoured, as observed in the fossil record in the last $3 \mathrm{My}$, it means that the fitness associated to the difference in advantages of the two traits is a monotonous function of the flow winding, and therefore either genetic variants progressing upwards on the head scale will still be favoured, or a local maximum is reached, by which evolution is arrested, in so far as the environment is not modified (in this case the brain of that 
specie is big enough, considering that the specie cannot afford having even smaller jaws).

This gives a strict scientific meaning to the observation by Darwin that there existed "mysterious laws of correlation" (ibid. Chap. VIII) in animal morphological evolution. Such correlations, happening so to speak "by the inside" of the developmental mechanics, are not sensitive to environmental changes, and they imply deterministic effects in evolution. Darwin himself, in his celebrated book, states that, when morphological variability is under the control of physical forces of morphogenesis, natural selection plays no role (Chap. IV).

One third and important reason that makes animal evolution somewhat predictable lies in the fact that the generic patterns are controlled by very few degrees of freedom, possibly only one (magnitude of the hyperbolic flow). Although such a parameter may vary randomly during evolution, a set of morphogenetic flows, which depend on only one degree of freedom, will be recurrent. Indeed, in traditional Darwinian evolution, shapes traits appear randomly. While this may be true in some respect (genetic mutations), randomness does not have the same consequences in a system with a high number of degrees of freedom, or only one. If a system has only one degree of freedom, random variations of the parameters (genotypes), map the system onto forms which are along the same path, back and forth (phenotypes). Stated mathematically, the mapping of genes onto forms is not bijective, not an isomorphism, but a projection onto a low dimensional variety.

If arrested at a given form, the next forms are upstream or downstream the existing flow lines, and these are repeatedly explored by modifications of the parameters. For example, animals with short or long legs, evolve from animals with long or short legs, and vice-versa. Legs cannot do anything else but elongate or shorten. If, at a given moment, having longer legs is favourable, then longer legs are selected. If they are not, longer legs will await a more favourable opportunity, but anyway, longer legs will always eventually recur. This is also true for necks, for tails, for skull volume, and even for head to pelvis distance, around a hyperbolic point. This is not contradictory with the genetic description in terms of Hox genes. Actually, the collinearity concept, introduced in genetic studies, makes the implicit assumption of a one dimensional geometry along the body axis (stated otherwise, it assumes that something like an axis exists for the body, without explaining how that axis is constructed during development). The reiteration of collinearities in appendages is rendered possible by the existence of twists and bendings of a physical process which produces lateral evaginations, folds, plates, etc. which locally break the symmetry of the 1D part. Each appendage has grossly a 1D character, and most of the morphogenetic-developmental-evolutionary difficulties arise in the area of the engulfments (hips and shoulder girdles, wrist bones) where the body parts are more 3D.

A possibility therefore appears, that the apparance of tetrapods be generic, and that it follows a general law with few degrees of freedom, although it has lots of genetic pa- rameters. In which case, Darwinian evolution plays with a very restricted set of shapes, with stringent internal (physical) correlations, and the known body forms might be unavoidable, in the long run.

\section{Glossary}

Advection. Transport of molecules may occur by diffusion, by active molecular transport, or be a mere transport by the tissue as it flows, like a log in a river. In this case, one speaks of advection by the flow.

Anlagen. A german word for the way biological structures, tissues, or bio-molecular fields are deposited and laid down.

Antero-posterior. From head to tail.

Apical ectodermal ridge. AER: a narrow thickening located along the edge of the limb bud, which is considered to drive the growth of the limb. It is recognizable on hands, as it spans eventually the area of the nails.

Autopod. The more distal segment of the limb (the hand in the case of humans).

Blastula. Name of the developing animal, at a stage where it is composed of a round mass of cells (from a few tens, to a few tens of thousands). At the blastula stage the living mass of cells is either a disc (as in birds) or round, as in mammals and amphibians. However, only a fraction of this mass of cells will truly form the embryo. A large part of the mass of cell will serve to form extra-embryonic organs such as the yolk-sac and the placenta. At the blastula stage, the mass of cells is generally composed of two layers of cells, partially delaminated.

Bauplan. A german word for the "blueprint" of the animals bodies. Whether this actually exists is debated. In effect, embryonic movements have a finite typology, therefore each generic attractor of the movement may correspond to a class of "blueprints" (for example: radiates or bilaterans), without understating any creator or "architect" for these blueprints.

Buckling. A non-linear physical phenomenon by which plates or sheets form stable wavy profiles under the action of in-plane stresses. Buckling is the natural explanation of most if not all biological folds, like brain convolutions, fingerprints, villi, neural crests, etc.

Chemotactism (chemotaxy). Tendency for cells to move up gradients of some chemical (signalling molecule). Chemotactism is related to a bias in filiopodia polymerization, in the presence of molecular gradients.

Cortical rotation. At the instant of fertilization, the spermatozoid triggers a calcium wave inside the ovocyte which provokes a rotation of the internal chemical content of the egg with respect to the outer shell (cortex). This is called cortical rotation. It is a physical phenomenon, which advects the entire content of the egg in a angular motion of approximately $30^{\circ}$, in the direction of the point of entry of the spermatozoid. This creates a symmetry-breaking in the egg, which acts on all constituents. The first cleavage line passes through the plane of asymmetry created by cortical rotation. The second cleavage is orthogonal to the 
first. This induces a global symmetry breaking forming a cross pattern, with a central point located caudally.

Collagen. A macromolecule which forms fibers and bundles of fibers in the body. It is the most important structural constituent of the body, forming up to $80 \%$ of the dry weight of tissues. All tissues contain collagen in an increasing concentration in the order: skin, muscle, tendon, ligament, cartilage, bone. The web of collagen may serve as guiding cue for cell migration.

Contralateral. The opposite side of an embryo. Quite often, developmental biologists do an experiment on one side only of an embryo, the other side serving as control.

Cytosol. The internal content of the cell, in between the membrane and the nucleus.

Deformation. The deformation is a tensor obtained from the displacement vector $\varepsilon_{i}$, by forming the terms $1 / 2\left(\partial_{i} \varepsilon_{j}+\partial_{j} \varepsilon_{i}\right)$. Deformed objects may exert forces (elastic forces), elastic objects displaced as a whole do not exert forces.

Digital arch. The set of all fingers plus the tissue in between, which form an arch which articulates to the carpal bones. The distribution of bones inside the digital arch is very simple, while the set of bones in the wrist is more complex.

Dimensionless equation. All dynamic equations, in particular the ones which govern the development of forms have a general "dimensionless" mathematical writing, which contains dimensioning constants. For example, gravitation law varies like 1 /square(distance) in dimensionless form, but there exists a weak dimensioning factor, called gravitational constant. In the case of fluid dynamics, the viscosity, the magnitude of the driving force and the size of the sample are general dimensioning parameters. The same dynamics, with different dimensioning constants may lead to similar patterns, albeit at different spatial or temporal scales.

Displacement. The vector $\varepsilon_{j}$ defined at each point by the local displacement, in a deformed configuration, with respect to a reference configuration (generally a stress free state). The final form during a dynamical process is obtained by the time integral of the displacement field. The displacement field is obtained from the equilibrium equation (Newton's law of dynamics).

Direct frog. Frogs which form normal legs, directly after the gastrulation motions, inside their egg. This is unlike normal frogs, which form legs after hatching, at the tadpole stage. Direct frogs have limb buds, bud do not show an Apical Ectodermal Ridge.

Distal. Located afar from the body, or from some center of growth: for example, the hand is more distal than the elbow, the marginal vein is more distal than the navel. Actually, the word "distal" is ill defined. From a physicist's point of view, it seems that a proper definition of "distal" would rather be, downstream the pressure gradient.

Dorsal. Related to the back. One should be aware that actually, vertebrates embryos form with the dorsal side upwards inside the egg. Te dorsal side of the blastula is the ectoblast, the ventral side the endoblast. The embryo is formed from folds of the upper tissue layers of the blastula, which close up on the ventral side (hence the presence of scar-lines on many individuals in the area of the belly).

Ectoderm. The upper layer of the embryonic sheets, at the stage where the embryo is composed of 3 layers (after gastrulation).

Ectopic. Ectopic grafts or expressions, consist in positioning a graft or a molecular source in an aberrant place, and watch the developmental outcome. For example, ectopic limbs can be obtained in aberrant places, by putting ectopically a bead of some chemical under the ectoderm, in that aberrant place.

Electroporation. A technique used to insert molecules inside embryonic tissues. Electrodes are used to generate local electric fields which render cell membrane porous; by these means signalling molecules or transcription factors can be introduced at ectopic places of the embryo. Electroporation experiments have a very low success rate, although they give spectacular results.

Embryo. Name of the developing animal, after the morula, blastula, and gastrula stages.

Endoderm. Lower embryonic sheet, at the stage when the embryo is composed of 3 layers (after gastrulation).

Engulfment. Embryogenesis of vertebrates truly starts when the number of cells reaches a value of approx. 50000. At this moment a complex vortex motion occurs. During this motion, the upper layer of cells, called epiblast rotates and dives underneath. During this motion the epiblast is twisted by a combination of rotation towards the median axis, vertical $U$ turn, and rotation away from the median axis, underneath. All this is often summarized as an "engulfment".

Epiblast. The upper layer of the presumptive embryonic sheets, at the stage where the presumptive embryo is composed of 2 layers (before gastrulation, at the blastula stage).

Epiboly. Movement of downwards curl and involution of the tissue layers during gastrulation. During the epiboly, the cells invaginate through a "hole" in the embryo upper layers. This hole is linear in the chicken, and called primitive streak.

Extra-cellular matrix. Material outside the cells, forming a net of macromolecules which serves as supporting medium for cell motion, and which is also an essential element of tissue stiffness, and orientational pattern.

Hox genes. Genes implicated in the actual forms of animal features. These genes are transcription factors, they code for proteins which interact with the DNA, therefore modifying the set of cellular actions.

Fibroblast growth factor. Fibroblast growth factors are molecules which (among other roles) maintain the proliferative activity of fibroblasts. There exist about 23 members of this family, with similar sequence. There exist 4 receptors of these growth factors. Fibroblasts are one of the major cell types. They provide the production of collagen and related supporting molecules. Hence FGFs and their receptors play a key role in many aspects of embryo development, angiogenesis, etc. FGFs also have chemotactant properties. 
Gastrulation. Complex movements by which a "formless" animal, acquires a recognizable shape. The movements start at the blastula stage, when the embryo is "formless", i.e. essentially round. These motions may include cellular flow and mechanical stretch and folding. The formation of the animal body is not a matter of a "prepattern" being drawn inside the ovocyte, and passed to the initially formless mass of cell. The formation of an animal is a combination of vortex flow and physical deformation of a flat layer.

Halteres. Set of appendages of the insects which look like a dwarf wing. These appendages serve to control the balance during flight.

Hensen's node. A small area located at the apex of the primitive streak which is considered as the "organizer" of embryo development. From a physicist's point of view this area is the front of a deformation wave, and not an "object" in itself.

Homeotic transformation. A transformation of the actual body of an animal, with respect to the normal body, obtained by knocking out, or over-expressing a given homeobox gene (in the entire animal body, not at an ectopic place). In many cases, homeotic transformations correspond to shifts along the body, in the direction of the colinearity axis.

Imaginal discs. Discs of cells (containing a few tens of thousands cells) from which insect appendages form. These groups of cells form at the boundaries of insect segments. They contain at first only a clutch of cells, which form a round mass, and progressively grows to form concentric rings. This rings form appendages by telescoping themselves outwards under the action of growth forces.

Invagination. A process of involution of tissue sheets by which the topology of a tissue sheet is changed from a sheet to a "sock".

Knock out. To knock out genes consists in generating an animal in which all alleles of a gene are absent. A mouse ko for a given gene will show traits related to the absence of that gene in the entire animal, and for the entire developmental process (unlike ectopic expressions, which show effects localized in time and space).

KRS: Koller Rauber Sickle. During avian and mammal morphogenesis, a region forms in the caudal part of the blastula which has the shape of a sickle (a crescent), and in which many molecular stainings are denser. This area seems to generate the vortex flow which is observed during gastrulation. The origin of this sickle may be tracked back to cell division anisotropies which accumulate a gradient of cells sizes forming a boundary along the sickle. This gradient may itself be tracked back to the early cell cleavage pattern which is asymmetrical (the cleavage line does not pass strictly through the middle of the first cells). This asymmetry is itself induced by the entry of the spermatozoid. The KRS is found roughly at the opposite of the entry point of the spermatozoid.

Limb plate. The area along the flanks from which limbs emanate. The limb plate is composed of ectoderm, mesoderm, and endoderm. It may have a complex structure, inherited from the embryo gastrulation movements.
Lateral plate. The entire flanks are "competent" to give limbs, from the pelvic area, up to the shoulder girdle area. This area is called lateral plate. It may correspond to the area where some fish have long fins. Somehow, the lateral plate gets segmented into limb plates.

Maternal proteins. The ovocyte is produced by a female. The proteins inside the ovocyte (or the insect syncitium), which are produced by the DNA of the mother are said to be maternal. Later during development, the embryo will produce its own proteins, with its own RNA.

Mesoderm. The middle layer of cells of an embryo, after gastrulation.

Morula. Very early stage of development at which the number of cells is less than about a hundred. At this stage, the mass of cells looks like a raspberry (morula in latin).

Neural crest. The folds of the embryo located along the backbone.

Poiseuille (Jean-Louis Marie). French medical doctor (1797-1869), who studied blood circulation, and who introduced the viscous flow in tubes, or between plates, the so-called "Poiseuille flow".

Poiseuille flow. The Poiseuille flow is a viscous flow between plates with a small spacing or inside a tube of small diameter. In such a geometry a lubrication approximation is done: fluid laminae are supposed to be parallel to the direction of the tube or plate. The velocity profile is found by considering the shear between these laminae as the most important. In this case, each lamina has a speed $V, V=0$ on the surfaces and is maximal in the center of the tube, or half-way between plates, and the profile of velocities is parabolic. This is a very good approximation for viscous fluids in thin films or capillary tubes, which are in contact with a more viscous fluid or a solid surface. It is used also to model flow in porous media. The Poiseuille profile is also used for open channel flows, and for elastic solids pushed between thin plates.

Potential vector. 2D flows are by definition "in plane". In such a case, the velocity vector can be analytically obtained from a scalar function, which is the third component (out-of-plane) of a so-called potential vector which is perpendicular to the plane of the flow. The in-plane velocity is the curl of the out-of-plane potential vector. The formalism is analogous to the electromagnetism.

Proximal. Opposite to distal (see distal).

\section{Koller's or Rauber's sickle. See KRS.}

Regulatory region. While genes code for proteins which may have a direct structural role (like collagen), other parts of the DNA may have a regulatory role for the genes. Modifying the regulatory region can affect the levels of expression of genes, without modifying the chemicals formulae of proteins.

Scaffold. Term used constantly by Darwin to describe the pattern of bones of animals. For Darwin, animals are obtained from each other, without "changing the scaffold" (e.g.: the bat forelimb has bones homologous to the mouse forelimb, but 500 times greater).

Rostro-caudal. From head to tail, generally synonym of antero-posterior, for animals which have a tail. 
Segmentation. A physico-chemical process by which uniform bodies acquire a segmented pattern (for example: formation of vertebrae along the backbone). The mechanism of segmentation is still debated. It contains molecular oscillations in the pattern of expression of growth factors, and physical movements of cells which orient themselves to form denser clusters separated by what will eventually be the segmentation furrow.

Sickle. Crescent area located at the caudal pole of the blastula which has attractive properties for cells. This crescent is inherited from the initial cortical rotation of the ovocyte, which is "blown up" by further cell divisions. Signaling molecule. A molecule which activates or inhibits cellular behaviours directly (not by changing the DNA expression pattern).

Somites. Segments along the dorsal area which are precursors of the vertebrae and other dorsal structures. There is a mismatch of $1 / 2$ wavelength between the somites and the final vertebrae distribution.

Sonic Hedge Hog. A gene coding for a protein which is a crucial signalling molecule, which plays a role in the pattern of fingers (among others).

Sphenoid bone. An important bone located at the base of the human head, which has been used as "tracer" of the morphogenetic movements by Dambricourt-Malassé. This bone shows a clear flexion during evolution, associated to a more pronounced forward winding of the head. This winding implies a contraction of the face, associated to a dilation of the cranium, hence a deterministic dynamical pattern of head formation ("inside story").

Stokes flow. Limiting case of the hydrodynamic equations, in which the inertial term is neglected, and only the viscous term is retained. It corresponds generally to very slow flows, in narrow regions. In general terms, solutions to the Stokes flow breakdown at some point where the conditions of the approximation is not satisfied. For example, in the case of vortices, the Stokes equations gives an infinite value of the speed of revolution in the core, which is non-physical (Stokes paradox). In the core of a vortex, the Stokes approximation breaks down, and actually, the core revolves as a solid rotation.

Stream function. A scalar function whose iso-values lines correspond to streamlines. It is the out of plane component of the potential vector.

Stress. A physical tensor, each term corresponding to the force exerted per unit surface on an elementary volume of matter, as considered in all its direction.

Stylopod. The segment of the limb between the shoulder and the elbow.

Syncitium. In insects, the cell nucleus of the early fertilized ovocyte will divide while the membrane will not. A large oval bag forms, containing many cell nuclei which are regularly organized (in stripes), although there is only one single cell membrane encapsulating all the nuclei (up to hundreds).

Texture. Internal correlations of a material, generally orientational correlations due to the existence of specific directions of growth which create a well recognizable tendency; the texture, defined as the orientation order pa- rameter is a quantitative notion. In the case of polycrystals, the texture is formed by the direction of growth of columnar single crystals which share a common axis of growth. In the case of biological tissue, the texture is related to the orientational order of macromolecules, such that growth is parallel to the local director of the macromolecules, and segmentation tends to be perpendicular or parallel to this director, hence forming regular patterns of segmented rays.

Transcription factor. A factor which modifies the pattern of expression of proteins of a cell, by binding to its DNA.

Tetrapod. Animal having four limbs (humans, horses, cats). Fish are not considered as tetrapods although they generally have 2 pelvic and two pectoral fins. Also, there exists a continuum of shapes between fish (sarcopterygian fish) and true tetrapods.

Variability. Word used by Charles Darwin often as a synonym for physical and chemical patterns of morphogenesis.

Ventral. Opposite to the dorsal side. The ventral side of the hand is the palmar side.

von Karman equation. When a flat plate is subjected to an in-plane stress, it tends to buckle, this is to say to deform in the perpendicular direction. The origin of this deformation is in the combination of in plane tensions, and out-of plane bending. Out-of-plane bending is associated to a force momentum, while in plane stress is associated to an elongation. The von Karman equation is the equilibrium equation which allows one to find the buckled state from the material constants and the compressive stresses. Buckling is invoked in biological folding for brain convolution development, fingerprints, neural crest formation, limb buds, intestine villi, etc.

Yolk-sac. An extra embryonic organ which has a circular shape. The yolk-sac is very well vascularized and connected to the embryo by a navel. The navel is formed actually of 2 halves, one left and one right half. In birds and reptiles, the yolk-sac serves to digest the yolk and bring back the fatty acids towards the embryo. In mammals, the yolk-sac often contributes to the placenta, by sticking itself to the mother uterus, instead of resting on a yolk. The lower part of the yolk-sac in contact with the yolk, has regular villosities, quite reminiscent of the villosities of the placenta in contact with the uterus.

Zeugopod. The second segment of the limb in tetrapods, from the elbow to the wrist.

\section{References}

1. V.E. Prince, Dev. Biol. 249, 1 (2002)

2. E.H. Davidson, D.H. Erwin, Science 311, 796 (2006)

3. V.F. Hinman, E.H. Davidson, PNAS 104, 19404 (2007)

4. A.C. McPherron, A.M. Lawler, S.-J. Lee, Nat. Genet. 22, 260 (1999)

5. A.S. Romer, Vertebrate Paleontology (The University of Chicago Press, Chicago, 1966) 
6. M. Krings, A. Stone, R.W. Schmitz, H. Krainitzki, M. Stoneking, S. Pääbo, Cell 90, 19 (1997)

7. N. Eldredge, S.J. Gould, phyletic gradualism in Models in Paleobiology, edited by T.J.M. Schopf (Freeman Cooper, San Francisco, 1972), pp. 82-115, reprinted in N. Eldredge, Time frames (Princeton Univ. Press., Princeton, 1985)

8. R. Dawkins, The Blind Watchmaker (W.W. Norton \& Company, Inc., New York, 1996)

9. M. Dennet, Darwin's Dangerous Idea: Evolution and the Meanings of Life (Simon and Schuster, 1995)

10. D'Arcy Wentworth Thomson, On growth and Form (Cambridge University Press, Cambridge, 1917)

11. S.J. Gaunt, Int. J. Dev. Biol. 44, 109 (2000)

12. C. Darwin, On the origin of species (Murray, London, 1859)

13. C. Darwin, On the origin of species (Murray, London, 1859), Chap. XIII. All further quotations of Darwin's work will appear in the text as (ibid., 1859), Chap. X

14. C.J. Cretekos, Y. Wang, E.D. Green, NISC Comparative Sequencing Program, J.F. Martin, J.J. Rasweiler IV, R.R. Behringer, Genes Dev. 22, 141 (2008)

15. L'origine de l'Humanité, edited by Y. Coppens, P. Picq (Fayard, Paris, 2000)

16. V. Fleury, Revue des Questions Scientifiques 177, 235 (2006)

17. G.A. Bellus, T.W. Hefferon, R.O. de Luna, J.T. Hecht, W.A. Horton, M. Machado, I. Kaitila, I. McIntosh, C.A. Francomano, Am. J. Hum. Genet. 56, $368(1995)$

18. R.J. Ferry Jr., M. Shim, topic Gigantism and Acromegaly e-medicine, July 2007, http://www . emedicine.com/ped/topic2634.htm

19. H. Lodish, A.B. Lawrence, S. Zipursky, P. Matsudaira, D. Baltimore, J. Darnell, Molecular Cell Biology (W.H. Freeman and Company, New York, 2000), Chap. 14

20. P.K. Dearden, M.J. Wilson, L. Sablan, P.W. Osborne, M. Havler, E. McNaughton, K. Kimura, N.V. Milshina, M. Hasselmann, T. Gempe, M. Schioett, S.J. Brown, C.G. Elsik, P.W.H. Holland, T. Kadowaki, M. Beye, Genome Res. 16, 1376 (2006), doi:10.1101/gr.5108606

21. L. Sanchez, D. Thieffry, J. Theor. Biol. 224, 517 (2003)

22. A central concept in developmental biology is that of a morphogen, a substance that specifies cell identity as a function of its concentration. A continuous gradient of morphogen concentration can elicit a set of unique cellular responses at a finite number of threshold concentrations: above the threshold, one response is elicited; below it, cells respond differently. In reference [19], Chap. 14

23. E. Farge, Curr. Biol. 13, 1365 (2003)

24. E. Honoré, F. Maingret, M. Lazdunski, A.J. Patel EMBO J. 21, 12 (2002)

25. S. Papageorgiou, Int. J. Dev. Biol. 50, 301 (2006)

26. W.J. Gehring, in Hox Gene Expression, edited by Dr. S. Papageorgiou (Landes Bioscience and Springer Science + Business Media, 2007), Chap. 1
27. G. Struhl, Nature 292, 635 (1981)

28. P.H. Lawrence, The making of a fly (Blackwell Publishing, Oxford, 1992)

29. L.I. Held Jr., Imaginal Discs, Developmental and Cell Biology Series (Cambridge University Press, Cambridge, 2005)

30. V. French, P.J. Bryant, S.V. Bryant, Science 193, 969 (1976)

31. J.H. Poslethwait, H. Schneiderman, Dev. Biol. 25, 606 (1971)

32. N. Serrano, P.H. O'Farrell, Curr. Biol. 7, R186 (1997)

33. J.A. Williams, S.W. Padock, S.B. Caroll, Development 117, 571 (1993), see also the interactive fly website: http://www.sdbonline.org/ $\mathrm{fly} /$ aimain/1aahome.htm

34. Branching in Nature, edited by V. Fleury, M. Leonetti, J.-F. Gouyet (EDP Sciences, Les Ulis, 2000)

35. T. Vicsek, Fractal Growth phenomena (World Scientific, Singapore, 2000)

36. P. Pelcé, Theory of growth and form in condensed matter Physics (Lavoisier, Paris, 2008)

37. V. Fleury, T. Watanabe, C.R. Biologies 327, 663 (2004)

38. J. Dumais, S.L. Shaw, C.R. Steele, S.R. Long, P.M. Ray, Int. J. Dev. Biol. 50, 209 (2006)

39. W. McGinnis, R.L. Garber, J. Wirz, A. Kuroiwa, W. Gehring, Cell 37, 403 (1984)

40. D. Duboule, Development (Suppl.) 120, 135 (1994)

41. D. Duboule, P. Dollé, EMBO J. 8, 1497 (1989)

42. B.G. Condie, M.R. Capecchi, Development 119, 579 (1993)

43. J. Charite, Cell 8, 589 (1994)

44. J. Zakany, D. Duboule, Curr. Opin. Genet. Dev. 17, 359 (2007)

45. F. Spitz, F. Gonzalez, D. Duboule, Cell 113, 405 (2003)

46. A.C. Burke, J.L. Nowicki, Am. Zool. 41, 687 (2001)

47. M. Coates, M. Cohn, BioEssays 20, 371 (1998)

48. M.J. Cohn, C. Tickle, Nature 399, 474 (1999); M. Cohn, K. Patel, R. Krumlauf, D.G. Wilkinson, J.D.W. Clarke, C. Tickle, Nature 387, 97 (1997)

49. R.C. Rallis, J.D. Buono, M.P.O. Logan, Development 132, 1961 (2005)

50. J.W.R. Schwabe, C. Rodriguez-Esteban, J.C. Izpisua-Belmonte, Trends Gen. 14, 229 (1998)

51. M.J. Cohn, J.C. Izpisúa-Belmonte, H. Abud, J.K. Heath, C. Tickle, Cell 80, 739 (1995)

52. K. Sekine, H. Ohuchi, M. Fujiwara, M. Yamasaki, T. Yoshizawa, T. Sato, N. Yagishita, D. Matsui, Y. Koga, N. Itoh, S. Kato, Nat. Genet. 21, 138 (1999)

53. A.M. Boulet, A.M. Moon, B.R. Arenkiel, M.R. Capecchi, Dev. Biol. 273, 361 (2004)

54. D.L. Chapman, N. Garvey, S. Hancock, M. Alexiou, S.I. Agulnik, J. Thomas, R.J. Bollag, L.M. Silver, V.E. Papaioannou, Dev. Dyn. 206, 379 (2000)

55. J.J. Gibson-Brown, S.I. Agulnik, D.L. Chapman, M. Alexiou, N. Garvey, L.M. Silver, V.E. Papaioannou, Mech. Dev. 56, 93 (1996)

56. C. Rallis, B.G. Bruneau, J.D. Buono, C.E. Seidman, J.G. Seidman, S. Nissim, C.J. Tabin, M.P.O. Logan, Development 130, 2741 (2003) 
57. C. Minguillon, J.D. Buono, M.P. Logan, Dev. Cell 8, 75 (2005)

58. P. Khan, B. Linkhart, H.-G. Simon, Dev. Biol. 250, 383 (2002)

59. J.K. Takeuchi, K. Koshiba-Takeuchi, K. Matsumoto, A. Vogel-Höpker, M. Naitoh-Matsuo, K. Ogura, N. Takahashi, K. Yasuda, T. Ogura, Nature 398, 810 (1999)

60. J. Zákány, D. Duboule, Cell Tissue Res. 296, 19 (1999)

61. R.D. Riddle, R.L. Johnson, E. Laufer, C. Tabin, Cell 75, 1401 (1993)

62. E. McGlinn, C.J. Tabin, Curr. Opin. Genet. Dev. 16, 426 (2006)

63. D. Brian, P. Harfe, J. Scherz, S. Nissim, H. Tian, A.P. McMahon, C. J. Tabin, Cell 118, 20 (2004)

64. J.W. Saunders, M.T. Gasseling, in EpithelialMesenchymal Interactions, edited by R.E. Billingham, R. Fleichmajer (Williams and Wilkins, 1968), pp. $78-97$

65. T. Mikiko, M.J. Cohn, P. Ashby, M. Davey, P. Martin, C. Tickle, Development 127, 4011 (2000)

66. M.K. Richardson, T.F. Carl, J. Hanken, R.P. Elinson, C. Cope, P. Bagley, J. Anat. 192, 379 (1998)

67. G.F. Oster, J.D. Murray, P.K. Maini, J. Embryol. Exp. Morph. 89, 93 (1985)

68. N.H. Shubin, P. Alberch, in Evolutionary Biology, edited by M.K. Hecht, B. Wallace, G.I. Prance (Plenum Press, New York, 1986), pp. 319-387

69. S. Rushikesh, M.F. Bastida, M. Ros, Dev. Biol. 310, 430 (2007)

70. J. Dubrulle, O. Pourquié, Curr. Opin. Genet. Dev. 12, $519(2002)$

71. C. Chiang, Y. Litingtung, M.P. Harris, B.K. Simandl, Y. Li, P.A. Beachy, J.F. Fallon, Dev. Biol. 236, 421 (2001)

72. D. Chourrout, F. Delsuc, P. Chourrout, R.B. Edvardsen, F. Rentzsch, E. Renfer, M.F. Jensen, B. Zhu, P. de Jong, R.E. Steele, U. Technau, Nature 442, 684 (2006)

73. D. Duboule, Science 266, 575 (1994)

74. B.I. Shraiman, PNAS 102, 8 (2005)

75. M.J. Cohn, Nature 406, 953 (2000)

76. L. Niswander, Int. J. Dev. Biol. 46, 877 (2002)

77. Y. Kawakami, J. Capdevila, D. Büscher, T. Itoh, N.J. Rodriguez-Esteban, J.C. Izpisúa-Belmonte, Cell 104, 891 (2004)

78. D.E. Rancourt, T. Tsuzuki, M.R. Capecchi, Genes Dev. 9, 108 (1995)

79. P. Agarwal, J.N. Wylie, O. Arkhito, C. Li, C. Deng, R. Grosschedle, B.G. Bruneau, Development 130, 623 (2003)

80. H. Popperl, H. Rikhof, H. Chang, P. Haffter, C.B. Kimmel, C.B. Moens, Mol. Cell 6, 255 (2000)

81. M.J. Cohn, K. Patel, R. Krumlauf, D.G. Wlkinson, J.D. Clarke, C. Tickle, Nature 387, 97 (1997)

82. E. Mooney, Lower Limb Embryology Review (2006), emedicine; http://www.emedicine.com/plastic/ topic215-htm
83. K. Kardong, Vertebrates: Comparative anatomy, function, evolution, 2nd edn. (McGraw-Hill, 1998)

84. S.C. Morris, Proc. Natl. Acad. Sci. USA 97, 4426 (2000)

85. D. Shu, X.-L. Zhang, S.C. Morris, Nature 384, 157 (1996)

86. S.C. Morris, The Crucible of Creation: The Burgess Shale and the Rise of Animals (Oxford University Press, New York, 1998)

87. D.-G. Shu, H.-L. Luo, S.C. Morris, X.-L. Zhang, S.X. Hu, L. Chen, J. Han, M. Zhu, Y. Li, L.-Z. Chen, Nature 402, 42 (1999)

88. A. Ritchie, Zool. J. Linn. Soc. 47, 69 (1967)

89. C.E. Thacker, Mol. Phyl. Evol. 26, 354 (2003)

90. P.J. Miller, J. Fish Biol. 5, 353 (2006)

91. E.B. Daeschler, N.H. Shubin, F.A. Jenkins Jr., Nature 440, 757 (2006)

92. D. Ahn, R.K. Ho, Dev. Biol. 322, 220 (2008)

93. C. Tabin, Development 116, 289 (1992)

94. J.A. Clarck, M.I. Coates, Bull. Mus. Nat. d'Hist. Nat., Paris, 4e série 17, 357 (1995)

95. H.M. Langevin, K.N. Storch, M.J. Cipolla, S.L. White, T.R. Buttolph, D.J. Taatjes, Histochem. Cell Biol. 125, 487 (2006)

96. S. Meier, D. Packard, Dev. Biol. 102, 309 (1984)

97. One finds many molecular stainings of the chorioamniotic fold in the data base GEISHA (Neuropillin, Fgfr2, etc.), http://geisha.arizona.edu/geisha

98. M.C. Davis, R.D. Dahn, N.H. Shubin, Nature 447, 473 (2007)

99. T.J. Sanger, J. Jeremy, J. Gibson-Brown, Evolution 58, 2103 (2004)

100. J.C. Rage, F. Escuillié, C.R. Acad. Sci. Paris Earth Sci. 330, 513 (2000)

101. H. Amasaki, H. Ishikawa, M. Daigo, Anat. Anz. 169, 145 (1989)

102. P. Falzoni, R. Boldorini, M. Zilioli, G. Sorrentino, Z. Minerva Ped. 47, 489 (1995)

103. http://www.cbc.ca/health/story/2007/11/06/ india-eight-limbs.html

104. J.S. Rackoff, The origin of the tetrapod limb and the ancestry of vertebrates, in The Terrestrial Environment and the Origin of Land Vertebrates, edited by A.L. Pachen (Academic Press, London, 1980)

105. M. Logan, C.J. Tabin, Science 283, 1736 (1999)

106. A. DeLaurier, R. Schweitzer, M. Logan, Dev. Biol. 299, 22 (2006)

107. C. Rodriguez-Esteban, T. Tsukui, S. Yonei, J. Magallon, K. Tamura, J.C.I. Belmonte, Nature 398, $814(1999)$

108. X. Liao, M.D. Collins, Dev. Dyn. 237, 1553 (2008)

109. J.C. Gerhardt, M. Danilchik, T. Doniach, S. Roberts, B. Rowning, R. Stewart, Development 107, 37 (1989)

110. C. Nouri, R. Luppes, A.E.P. Veldman, J.A. Tuszynski, R. Gordon, Phys. Biol. 5, 1 (2008)

111. S. Gilbert, Developmental Biology (Sinauer Associates, MA, 2000) 
112. H. Driesch, in Foundations of Experimental Embryology, edited by B.H. Willier, J.M. Oppenheimer (Hafner, New York, 1892)

113. L. Chabry, Embryologie normale et tératologique des Ascidies, edited by F. Alcan (Paris, 1887)

114. Gastrulation, edited by C. Stern (Cold Spring Harbor Laboratory Press, New York, 2004)

115. M.C. Lane, M.D. Sheets, Dev. Biol. 296, 12 (2006)

116. W. Kimura, S. Yasugi, C.D. Stern, K. Fukuda, Dev. Biol. 289, 283 (2006)

117. K. Joubin, C.D. Stern, Cell 98, 559 (1999)

118. O. Spemann, H. Mangold, Entwicklungsmech. Org. 100, 599 (1924); Translated into English by V. Hamburger, in: Foundations of Experimental Embryology, edited by B.H. Willier, J.M. Oppenheimer (Prentice Hall International, Englewood Cliffs, NJ, 1924)

119. V. Fleury, A. Al-Kilani, M. Unbekandt, T.-H. Nguyen, Organogenesis 3, 49 (2007)

120. R. Keller, Science 298, 1950 (2002)

121. C. Cui, X. Yang, M. Chuai, J.A. Glazier, C.J. Weijer, Dev. Biol. 284, 37 (2005)

122. R. Keller, J. Shih, A. Sater, Dev. Dyn. 193, 199 (1992)

123. R. Keller, L. Davidson, A. Edlund, T. Elul, M. Ezin, D. Shook, P. Skoglund, Philos. Trans. R. Soc. Lond. Biol. Sci. 355, 897 (2000)

124. C.D. Stern, O. Voiculescu, F. Bertocchini, I. Skromne, R.E. Keller, Nature 306, 318 (2007)

125. N. Rivier, M.F. Miri, C. Oguey, Coll. Surf. A 263, 39 (2005)

126. C. Odin, T. Guilbert, A. Al-Kilani, O.P. Boryskina, V. Fleury, Y. Le Grand, Opt. Expr. 16, 16151 (2008)

127. B. Wojciak-Stothard, A.J. Ridley, J. Cell Biol. 161, $429(2003)$

128. S. Munevar, Mechanics of Fibroblast Migration: a Dissertation, UMMass Medical school (printed by scholarship@UMMS 2003), and references therein

129. R. Wetzel, Arch. Entw. Mech. Org. 119, 188 (1929)

130. M. Callebaut, E. Van Nueten, H. Bortier, F. Harrisson, J. Morphol. 255, 315 (2003)

131. V. Fleury, Organogenesis 2, 6 (2005)

132. X. Yang, D. Dormann, A.E. Münsterberg, C.J. Weijer, Dev. Cell 3, 425 (2002)

133. J. Painter, O.K. Maini, Bull. Math. Biol. 62, 501 (2000)

134. S.C. Chapman, F.R. Schubert, G.C. Schoenwolf, A. Lumsden, Dev. Biol. 245, 187 (2002)

135. N.H. Patel, Science 266, 581 (1994)

136. F. Bertocchini, C.D. Stern, Dev. Cell 3, 735 (2002)

137. F. Graner, J.A. Glazier, Phys. Rev. Lett. 69, 2013 (1992)

138. M. Chuai, C.J. Weijer, Curr. Top. Dev. Biol. 81, 135 (2008)

139. Int. J. Dev. Dyn., Special issue Developmental Morphodynamics 50, (2006)

140. A.G. Jacobson, R. Gordon, J. Exp. Zool. 197, 2 (1976)

141. F. LeNoble, D. Moyon, L. Pardanaud, L. Yuan, V. Djonov, R. Mattheijssen, C. Bréant, V. Fleury, A. Eichmann, Development 131, 361 (2004)
142. M. Unbekandt, P.M.D. Moral, F. Sala, S. Bellusci, D. Warburton, V. Fleury, Mech. Dev. 125, 314 (2008)

143. A.M. Malek, G.H. Gibbons, V.J. Dzau, S. Izumo, J. Clin. Invest. 92, 2013 (1993)

144. C.S. Muratore, H.T. Nguyen, M.M. Ziegler, J.M. Wilson, J. Pediatr. Surg. 35, 906 (2000)

145. M. Théry, A. Jiménez-Dalmaroni, V. Racine, M. Bornens, F. Jülicher, Nature 447, 7143 (2007)

146. P. Roy, Z. Rajfur, P. Pomorski, K. Jacobson, Nat. Cell Biol. 4, E91 (2002)

147. D.J. Tschumperlin, G. Dai, I.V. Maly, T. Kikuchi, L. Laiho, A.K. Mc Vittie, K.J. Haley, C.M. Lilly, P.T. So, D.A. Lauffenburger et al., Nature 429, 83 (2004)

148. A. Al-Kilani, S. Lorthois, T.-H. Nguyen, F. Le Noble, A. Cornelissen, M. Unbekandt, O. Boryskina, L. Leroy, V. Fleury, Phys. Rev. E 77, 1 (2008)

149. C.-M. Lo, H.-B. Wang, M. Dembo, Y.-L. Wang, Biophys. J. 79, 144 (2000)

150. K.A. Lazopoulos, D. Stamenovic, J. Biomech. 41, 1289 (2008)

151. T.-H. Nguyen, A. Eichmann, F. Lenoble, V. Fleury, Phys. Rev. E 73, 061907 (2006)

152. G. Forgacs, R.A. Foty, Y. Shafrir, M.S. Steinberg, Biophys. J. 74, 2227 (1998)

153. K. Aderogba, J. Eng. Math. 10, 143 (1976), and references therein

154. E. Guyon, J.-P. Hulin, L. Petit, C.M. Mitescu, Physical Hydrodynamics (Oxford University press, Oxford, 2001)

155. U.S. Schwarz, S.A. Safran, Phys. Rev. Lett. 88, $048102(2002)$

156. R. Keller, L. Davidson, in Gastrulation from Cells to Embryos, edited by C. Stern (Cold Spring Harbor Press, New York, 2004)

157. C. Niehrs, H. Steinbeisser, E.M. De Robertis, Science 263, 817 (1994)

158. E.A. Zamir, A. Czirók, C. Cui, C.D. Little, B.J. Rongish, Proc. Natl. Acad. Sci. USA 103, 52 (2006)

159. M. Chuai , W. Zeng, X. Yang, V. Boychenko, J.A. Glazier, C.J. Weijer, Dev. Biol. 296, 137 (2006)

160. C.-H. Lee, B.M. Gumbiner, Dev. Biol. 171, 363 (1995)

161. J.-I. Sakiyama, A. Yamagishi, A. Kuroiwa, Development 130, 1225 (2003)

162. T. Mikawa, A. Poh, K. Kelly, Y. Ishii, D. Reese, Dev. Dyn. 229, 422 (2004)

163. A.B. Verkhovski, T.M. Svitkina, G.G. Borisy, Curr. Biol. 9, 11 (1999) (in this remarkable piece of work, the authors show that it suffices to gently push a cell on one side to generate a massive directional motion of the cell, this means that cells react to a presure gradient by a directional move oriented by the gradient)

164. I. Skromme, C. Stern, Development 128, 2915 (2001)

165. D.L. Chapman, N. Garvey, S. Hancock, M. Alexiou, S.I. Agulnik, J. Thomas, R.J. Bollag, L.M. Silver, V.E. Papaioannou, Dev. Dyn. 206, 379 (1996)

166. J.-A. Montero, C.-P. Heisenberg, Trends Cell Biol. 14, $620(2004)$

167. H.B. Adelmann, Q. Rev. Biol. 11, 284 (1936) 
168. M. Saha, M. Servetnick, R. Grainger, Curr. Opin. Genet. Dev. 2, 582 (1992)

169. F. Marlow, F. Zwartkruis, J. Malicki, S.C.F. Neuhauss, L. Abbas, M. Weaver, W. Driever, L. Solnica-Krezel, Dev. Biol. 203, 382 (1998)

170. D. Stopak, N.K. Wessels, A.K. Harris, Proc. Natl. Acad. Sci. USA 82, 2804 (1985)

171. V. Krenn, F. Wachtler, Anat. Embryol. 181, 453 (1990)

172. G. Kardon, Development 125, 4019 (1998)

173. O.P. Boryskina, V. Fleury, abstract of the VII Kharkiv Young Scientist conference, Ukraine National Academy of Science, Institute of radiophysics and Electronics, 2008; O.P. Boryskina, Y. Le Grand, C. Odin, V. Fleury, J. Eur. Microw. Association 4, 255 (2008)

174. B.J. Damon, V. Mezentseva, J.S. Kumaratilake, G. Forgacs, S.A. Newman, Dev. Biol. 321, 319 (2008)

175. O. Pourquié, Ann. Rev. Cell Dev. Biol. 17, 311 (2001)

176. A.G. Jacobson, in The origin and fate of somites, edited by E.J. Sanders (IOS Press, Amsterdam, 2001)

177. B.H. Lipton, A.G. Jacobson, Dev. Biol. 38, 91 (1974)

178. S. Meier, A.G. Jacobson, J. Exp. Zool. 219, 217 (1982)

179. P.L. Gould, Analysis of Plates and Shells (Prentice Hall, 1999)

180. M. Kücken, Forensic Sci. Int. 171, 85 (2007)
181. M.J. Garcia-Garcia, J.T. Eggenschwiler, T. Caspary, H.L. Alcorn, M.R. Wyler, D. Huangfu, A.S. Rakeman, J.D. Lee, E.H. Feinberg, J.R. Timmer, K.V. Anderson, Proc. Natl. Acad. Sci. USA 102, 5913 (2005)

182. K. Shariff, A.A. Leonard, Annu. Rev. Fluid. Mech. 24, 235 (1992)

183. H. Mae-Wan, J. Haffegee, R. Newton, Z. Yu-ming, J.S. Bolton, S. Ross, Bioelectrochem. Bioenerg. 41, 81 (1996)

184. J.W. Freeman, F.H. Silver, Connect. Tissue Res. 456, 107 (2005)

185. W. Wilson, N.J. Driessen, C.C. van Donkelaar, K. Ito, Osteoarthritis Cartilage 14, 1196 (2006)

186. G. Karsenty, E.F. Wagner, Dev. Cell 2, 389 (2002), and references therein

187. R. Amprino, Anatom. Embryol. 172, 1 (1985)

188. S. Meier, J. Embryol. Exp. Morph. 55, 291 (1980)

189. M.A. England, in Somites in developing embryos, edited by R. Bellairs, D.A. Ede, J.W. Walsh (Plenum Press, New York, 1986)

190. A. Dambricourt-Malassé, C.R. Palevol. 5, 109 (2006)

191. A. Dambricourt-Malassé, M.-J. Deshayes, F. Cahiers d'anthropologie et de biométrie humaine 23, 1 (2005)

Note from the publisher: Figures 1, 3-24, 31, 34, 40, 44: All rights reserved. 